\title{
POSITRON ANNIHILATION IN METALS \\ AT VERY HIGH PRESSURES
}

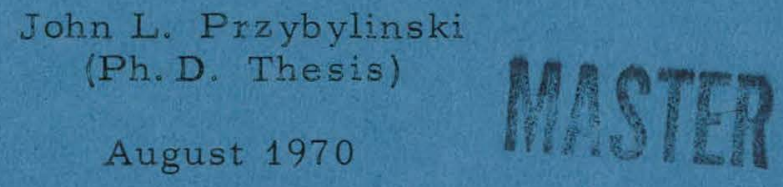

AEC Contract No. W - 7405-eng -48
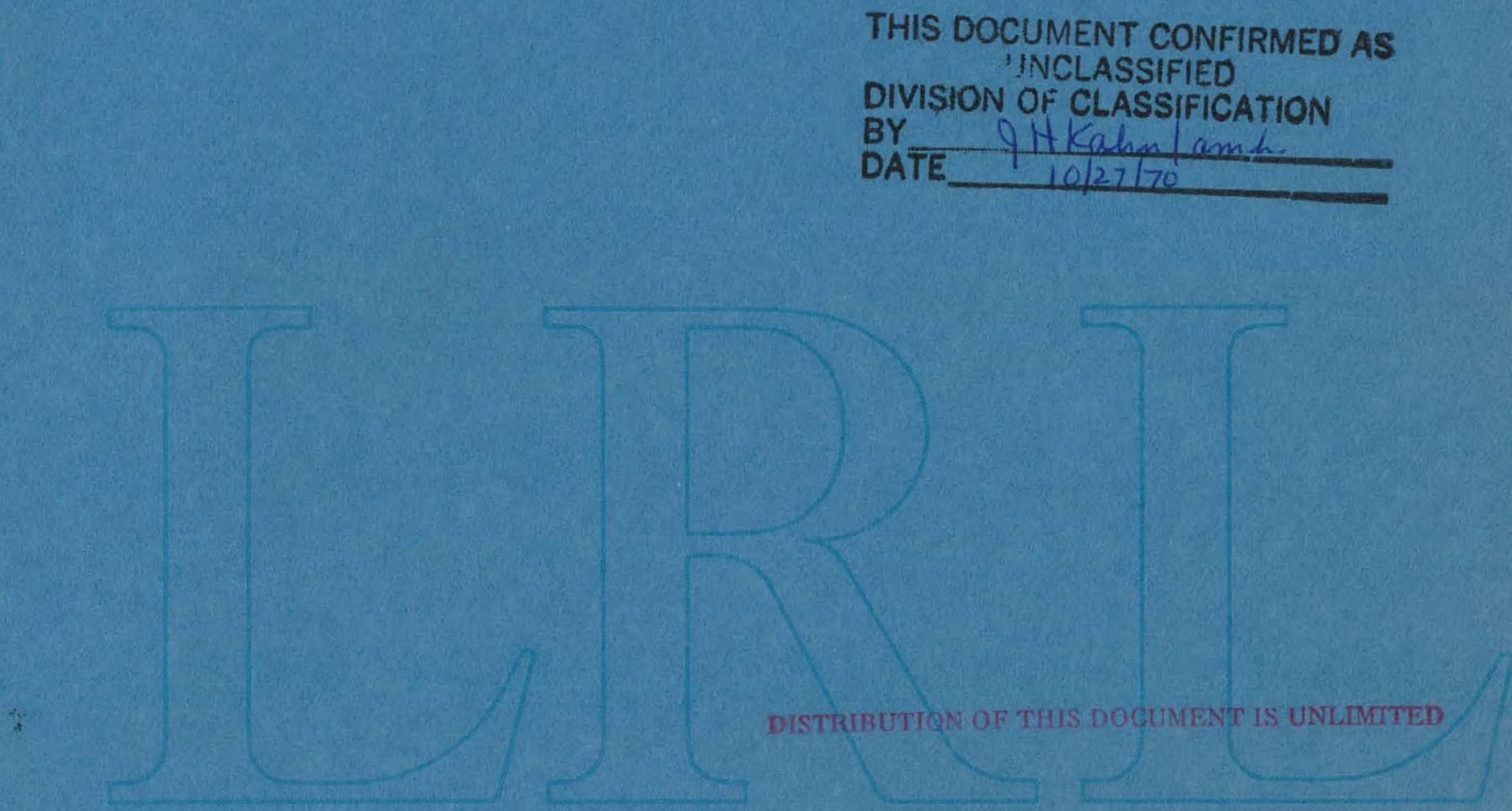

LAWRENCE RADIATION LABORATORY 


\section{DISCLAIMER}

This report was prepared as an account of work sponsored by an agency of the United States Government. Neither the United States Government nor any agency Thereof, nor any of their employees, makes any warranty, express or implied, or assumes any legal liability or responsibility for the accuracy, completeness, or usefulness of any information, apparatus, product, or process disclosed, or represents that its use would not infringe privately owned rights. Reference herein to any specific commercial product, process, or service by trade name, trademark, manufacturer, or otherwise does not necessarily constitute or imply its endorsement, recommendation, or favoring by the United States Government or any agency thereof. The views and opinions of authors expressed herein do not necessarily state or reflect those of the United States Government or any agency thereof. 


\section{DISCLAIMER}

Portions of this document may be illegible in electronic image products. Images are produced from the best available original document. 


\section{PAGES $\mathrm{i}$ to $\mathrm{ii}$ WERE INTENTIONALLY LEFT BLANK}


LEGAL NOTICE

This report was prepared as an account of work sponsored by the United States Government. Neither the United States nor the United States Atomic Energy Commission, nor any of their employees, nor any of their contractors, subcontractors, or their employees, makes any warranty, express or implied, or assumes any legal liability or responsibility for the accuracy, completeness or usefulness of any information, apparatus, product or process disclosed, or represents that its use would not infringe privately owned rights.

\section{Contents}

ABSTRACT

I. POSTRRON ANNIHTLATION

A. Introduction

B. Theory

1. Free Electron Theory

2. Real Metals -

3. Positron Annihilation - 11

C. Mathematicall Methods

1. ( $p)$ and $n(p)$ from $I\left(p_{z}\right)$

2. Effect of Temperature

3. Number of Electrons Annihilating

4. Relative Widths of Curves

D. Experimental

1. High Pressure Apparatus - 3.3

2. Range of Positrons

3. Angular Correlation Apparatus

E. Results and Discussion

1. Aluminum - 48

2. Bismuth

3. Ytterbium

II: Mössbuuer Effect in by 161

A. Introduction - 93

B. Theory - 93

C. Experimential -m-and 98

D. Results and Discussion 105 


$$
-i v-
$$

III. CRYSTALIINE PHOSPHOROUS

A. Introduction

B. Theory - 111

C. Results (-. 113

D. Discussion - 119

ACKNOWLEDGMENTS -

REFERENCES - 


\title{
POSITRON ANNIHILATION IN METALS AT VERY HIGH PRESSURES
}

John L. Przybylinski

Inorganic Materials Research Division, Lawrence Radiation Laboratory, Department of Chemistry, University of California, Berkeley, California

\begin{abstract}
The study of the angular correlation of gamma rays from positrons annihilating with electrons in metals can be used as a tool in the investigation of the momentum distribution of conduction electrons in a metal. This method was used in the study of three metals, aluminum, bismuth, and ytterbium, at pressures up to. 100 kilobars. Aluminum yielded unexpected and only partially explained results. The unusual results are thought to be associated with the state of strain of the aluminum specimen. The bismuth results were in agreement with the Free Electron Model. The results on ytterbium were most interesting. They provided the first confirmatory evidence of a proposed promotion of an electron from a $4 f$ state to a $5 \mathrm{~d}$ conduction band at high pressures. Surprisingly, the promotion appears to take place before the phase transition from fcc to bcc at $40 \mathrm{kbar}$. Ytterbium in the $\mathrm{fcc}$ phase above $20 \mathrm{kbars}$, therefore, cannot be considered to be a semiconductor unless the 5 d electrons are considered as core electrons for the purpose of conductivity.

The Mössbauer effect of $\mathrm{Dy}^{161}$ in gadolium metal at very high pressures was measured. The experiment failed to confirm earlier work on the same system by Stone. However, it was consistent with work in other laboratories on similar systems.

The resiatance of crystalline phosphorous was carefully measured from $60 \mathrm{kbar}$ to $130 \mathrm{kbar}$. These measurements persuasively indicated that
\end{abstract}




$$
-v i-
$$

the phase transition from rhombehedral to simple cubic which was reported by Jamieson to occur at $111 \pm 9$ kbar does not occur below $130 \mathrm{kbar}$ on the pressure scale that was used here. 


\section{POSIIRON ANNIHTHATION}

\section{A. INTRODUCTION}

The understanding of the electronic structure of metals is fundamental to the understanding of the characteristic properties of metals. The characteristic properties of metals are due to the unusual "freedom" of some of the electrons in the metal. If the electronic structure of metals is to be understood it is necessary to derive theories which are concerned with electrons in metals and compare the results of these theories with experiment. Virtually all theories of electrons in metals involve the concentration of free or ccinduction electrons in metals as a parameter. A fundamental test of a theory can then be made by altering the concentration of electrons in the metal. Since the number of free electrons in a given metal can not generally be altered, the volume of the metal must be altered in order to affect a change in the concentration of electrons.

There are two primary methods that can be used to affect a volume change; the temperature or the pressure can be changed. Of these two methods, the application of pressure causes a much larger change in volume.

The kinds of experiments that can be done at very high pressures are severly limited by the requirements of the high pressure apparatus. Most experiments which yield information about the electron energy states of the conduction electrons in a metal require that the electrons have long mean free paths. This in turn requires that very pure single crystal samples be used at very low temperatures. These conditions 
are, unfortunately, not compatible with very high pressures at the present time. The use of single crystals requires that truly hydrostatic pressures be used. If hydrostatic pressures are not used, there is a strong possibility that portions of the crystal will recrystallize in new orientations in order to minimize the strain energy of the specimen. This is due to pressure gradients and shear forces in the system. Hydrostatic conditions have reportedly been obtained at pressures as high as $60 \mathrm{kbars},{ }^{\perp}$ at room temperature. 'Ihe limit at IIquid helium temperatures is much lower; it does not exceed a few kbars. This is principaljy due to the lack of fluid media to transmit the pressure. Even elemental nitrogen solidifies at about $30 \mathrm{kbars}$ at room temperature. $^{2}$

The types of experiments which can be performed to the high pressure limits of static high pressure experiments are limited by practical considerations to experiments which can be performed at room temperature, or to experiments whose results are not strongly ef'lected by the state of strain of the material under study. Electrical resistance measurements, for instance, are easily performed at very high pressures. However, the experiment does not yield much fundamental information because of the complex dependence of the resistivity phenomenon on basic parameters.

Study of the angular correlation of gamma rays from positrons annihilating with electrons in a metal, on the other hand, is an appealing experiment to perform at very high pressures. 'l'he data can be interpreted to give information about the momenta of the free electrons in a metal. The experiment does not require very pure 
specimens or low temperatures. Finally, the $0.511 \mathrm{MeV}$ gamma rays which are produced by the annihilation event can easily penetrate a moderate mass, such as a high pressure cell.

It is the study of the angular correlation of annihilation gamma rays which was used to obtain the data presented herein. Three different metals were chosen for the experiment for three different reasons.

Aluminum was chosen as an example of a "normal" metal in order to compare the predicted change of the Fermi momentum as a function of pressure with experiment; and to compare the results on our new higher resolution instrument with previous results on a low resolution instrument. 3

Bismuth was chosen because it exhibits several high pressure phases which are associated with large changes in the electrical conductivity. This experiment was performed to see if there are large scale changes in the Fermi surface at these transitions.

Ytterbium was chosen because of the postulated promotion of an electron from a $4 \mathrm{f}$ state to a $5 \mathrm{~d}$ state at the phase transition from fec to bcc. ${ }^{4-6}$. This thansition occurs at about $40 \mathrm{kbar}$ at room temperature. 50,51 If the postulated promotion did occur it would have a very large effect on the positron annihilation spectrum. This experiment would constitute the first direct test of the theorized transition. 


\section{B. THEORY}

\section{Free Electron Theory.}

The study of the Angular Correlation of Gamma Rays which are emitted when a positron annihilates with an electron in a metal is a tool which can be used to study the distribution of momentum states of conduction electrons in a metal. In order to understand the results of such an experiment, it is first necessary to understand what the expected distribution of momentum states is for various metals. With this in mind, some elementary Solid State Physics will be discussed first, beginning with the free electron theory of metals.

Consider a cube of metal with side length $\mathrm{L}$, volume, $\mathrm{V}=\mathrm{L}^{3}$. If the conduction electrons in this piece of metal are considered to be under a constant zero potential, the single electron Schroedinger equation becomes,

$$
-\frac{m^{2}}{2 m} \nabla^{2} \psi(\vec{r})=E \psi(\vec{r})
$$

The solutuions of this equation normalized to the volume, $V$, are

$$
\therefore(\overrightarrow{\mathrm{R}})=(\hat{\mathrm{l}} / \mathrm{v} \cdot)^{3 / \mathrm{L}} \cdot \exp (\mathrm{i} \overrightarrow{\mathrm{k}} \cdot \overrightarrow{\mathrm{r}})
$$

The varitousisolutions are labeled with the wavevectors, $\vec{k}$.

Boundary conditions must now be imposed on these solutions. I'he often used boundary conditions, which are those of requiring the wavefunctions to become zero with zero slope at the boundaries, are unsatisfactory. This is because it is desired to consider only states in the bulk of the metal. The boundaries, therefore, can not be considered explicilly. Considering a cube of infinite size is also 
unsatisfactory because of mathematical difficulties. If, however, the cube is considered to be bend around until two opposite faces are joined together, one pair of boundaries is eliminated. Mathematically it is possible to envision doing this with all three pairs of sides simultaneously to generate an unbounded finite solid. After traversing a distance $\mathrm{L}$ in the $\mathrm{x}, \mathrm{y}$, or $\mathrm{z}$ direction, the wavefunction in such a solid must be identical to the original wavefunction. The boundary conditions then become the so-called periodic boundary conditions.
\end{abstract} For the $\mathrm{x}$ direction, then, $\psi_{\mathrm{R}}(\vec{x})=\psi_{\mathrm{k}}\left(\overrightarrow{\mathrm{x}}^{+} \hat{\mathrm{j}}_{\mathrm{x}} \mathrm{L}\right) \therefore$ The vector $\hat{\mathrm{j}}_{\mathrm{x}}$.!is a unit vector in the $\mathrm{x}$ direction. This yields the equation

$$
\exp (i \vec{k} \cdot \vec{r})=\exp \left(i \vec{k} \cdot\left(\vec{r} \hat{j}_{x} L\right)\right)=\exp (i \vec{k} \cdot \vec{r}) \exp \left(i k_{x} L\right)
$$

Therefore, $\exp \left(i k_{x} L\right)=1$. This occurs when $k_{x} L=2 \pi n$. $n$ is an integer.

Each of the components of the wavevector, $\vec{k}=\hat{j}_{x} k_{x}+\hat{j}_{y} k y=\hat{j}_{z} k z$, must satisfy the relationship $k_{x}, k_{y}, k_{z}=0 ; \pm 2 \pi / L ; \pm 4 \pi / L ; \ldots \ldots .$. The numbers $k_{X}, k_{y}, k_{z}$, along with the spin quantum number, in $s$, are the quantum numbers for the problem. Although $k$ is quantized, it is usually discussed as if it were continuous. This is because of the very close spacing of the states for crystals of macroscopic size.

Using the linear momentum operator, $-i k \vec{\nabla}$, the linear momentum, $\vec{p}$, is found.

$$
\begin{aligned}
\overrightarrow{\mathrm{p}} \psi_{\overrightarrow{\mathrm{k}}}(\overrightarrow{\mathrm{r}}) & =-i \hbar \vec{\nabla}(1 / V)^{1 / 2} \exp (-i \overrightarrow{\mathrm{k}} \cdot \vec{r}) \\
& =\pi k \psi_{\overrightarrow{\mathrm{k}}}(\overrightarrow{\mathrm{r}}) \\
\overrightarrow{\mathrm{p}} & =\hbar \overrightarrow{\mathrm{k}} .
\end{aligned}
$$

The momentum is lintarly related to the wavevector and is independent of position in the crystal. 
Similarly for the energy,

$$
\begin{gathered}
-\frac{\hbar^{2}}{2 m} \nabla_{\vec{k}}^{2}(\vec{r})=\frac{\hbar^{2} k^{2}}{2 m} \psi_{\vec{k}}(\vec{r}) \\
E_{k}=\hbar^{2} k^{2} / 2 m
\end{gathered} .
$$

Thus the energy has a simple quadratic dependence on the wavevector.

The energy of the highest occupied state at $0^{\circ} \mathrm{K}$ is called the Fermi energy, $E_{f^{\circ}}$ Associated with this energy is a Fermi wavevector, $\mathrm{kf}_{f^{*}} \mathrm{E}_{\mathrm{f}}=\mathrm{kn}^{2} \mathrm{k}_{\mathrm{f}}^{2} / 2 \mathrm{~m}$. The Fermi momentumi is similarly derined. An examination of the allowed values of $\vec{k}$ reveals that there is one allowed value of $\vec{k}$ for each volume element $(2 \pi / L)^{3}$ of wavevector space. There are, then, two allowed states, $m_{s}= \pm 1 / 2$, for each volume element $(2 \pi / L)^{3}$ of wavevector space. The density of states in wavevector space, $\omega(k)$, is $2 /(2 \pi / L)^{3}$. There are $N$ conduction electrons in the metal. The total volume occupied in wavevector space is $4 / 3 \pi \mathrm{k}_{\mathrm{f}}^{3}$. The number of eloctrons mukt equal the occupicd volume in wavevcctor apace time 3 the density of states. Thus

$$
\begin{aligned}
& N=\frac{4}{3} \pi k_{f}^{3} \cdot 2 /(2 \pi / L)^{3}=\frac{V}{3 \pi^{2}} k_{f}^{3} \\
& k_{f}=\left(3 \pi^{2} N / V\right)^{1 / 3}
\end{aligned}
$$

The Ferm 1 momentum and energy may be derived f'rom this

$$
\begin{aligned}
& p_{f}=\pi k_{f}=\hbar\left(3 \pi^{2} \mathrm{~N} / \mathrm{v}\right)^{1 / 3} \\
& \mathrm{E}_{f} \because \frac{\pi^{2} k_{f}^{2}}{2 m}=\frac{\hbar^{2}}{2 m}\left(3 \pi^{2} \mathrm{~N} / \mathrm{V}\right)^{2 / 3}
\end{aligned}
$$


Note that although the density of states depends on the dimensions of the crystal, the maximum occupied energy state depends only on the concentration of electrons. This concentration may be varied by changing the number of conduction electrons or the volume of the metal. The number of conduction electrons can not generally be changed. The volume, however, can be changed a significant amount for nearly all metals by the application of very high pressures.

\section{Real Metals}

The above discussion assumed that the conduction electrons in a metal experience zero potential throughout the metal. A little thought reveals that this cannot be true. There must, for example, be near singularities in the potential at the nucleus. In addition the proper wavefunctions must be orthogonal to the occupied core states. If the free electron plane wave functions are represented by $\psi_{\vec{k}}$, a new set of wavefunctions, $\underset{\overrightarrow{\mathrm{k}}}{\Psi}$, may be constructed using these functions and the properly orthogonalized set of core states, $\phi_{i}$. These are

$$
\underset{\vec{k}}{\Psi}=\psi_{\vec{k}}-\sum_{i} \phi_{i}^{*} \int \psi_{\vec{k}} \phi_{i} d \tau
$$

These new wavefunctions can be seen by inspection to be orthogonal. to all of the core states. It happens that these wavefunctions, which are known as orthogonalized plane waves (OPW), are also excellent approximations to the true wavefunctions over a large fraction of wavevector space.

In the interatomic spaces all the $\phi_{i}$ are very small and $\underset{\vec{k}}{\Psi}$ will be closely equal to $\psi_{\vec{k}}$. It is the behavior of the conduction electrons in these interatomic spaces that largely determines many metallic 
properties. The free electron plane wave states are pseudowavefunctions. The pseudopotentials associated with them may be nearly zero. The pseudopotentials are the result of considering the energy of the coreelectron-like part of the OPW to be combined with the true potential to form a new potential. The true potential and this energy nearly cancel out.

The pseudowavefunctions of electrons in real metals are not pure free electron plane waves. The lattice has an effect on the electrons so that the Schroedinger equation becomes

$$
-\frac{\hbar^{2}}{2 m} \nabla^{2} \psi+U(\vec{r}) \psi=E \psi \text {. }
$$

$U(\vec{r})$ is an appropriate pseudopotential with the periodicity of the lattice. The snlutions to this equation may be written in the form

$$
\psi_{\vec{k}_{0}}=\sum_{m} C_{m} \exp \left(-i \vec{k}_{m} \cdot \vec{r}\right)
$$

The sum is over all reciprocal lattice vectors, $\vec{G}_{I I} \cdot \vec{k}_{I I}=\vec{k}_{U}-\vec{G}_{I n}$. If $U(\vec{r})$ is small it may be considered to be a perturbation on the free electron wavefunctions and the $\psi$ can still generally be labeled by a wavevector $\overrightarrow{\mathrm{k}}_{0}$ which is equal to the $\overrightarrow{\mathrm{k}}_{11}$ which corresponds to the dominant term in the expansion. That is $C_{n}^{2} \gg C_{m}^{2}, m \neq n$. The result of this pseudopotential is to cause discontinuities in the energy when $\mathrm{k}_{U}=\frac{1}{2} \mathrm{G}_{11}$. This is the origin of band gaps. This is illustrated in Fig. I for a one-dimensional lattice, with lattice constant a. The dashed curve in the figure i.s the hehavior expested for pure free electrons. It is shown for comparison.

In the case when $k_{0}^{2}=k_{m}^{2}$ which occurs when $\vec{k}_{0}=\frac{1}{2} \vec{G}_{\text {III }}$ simple perturbation theory breaks down. For this particular case two waverunctions 


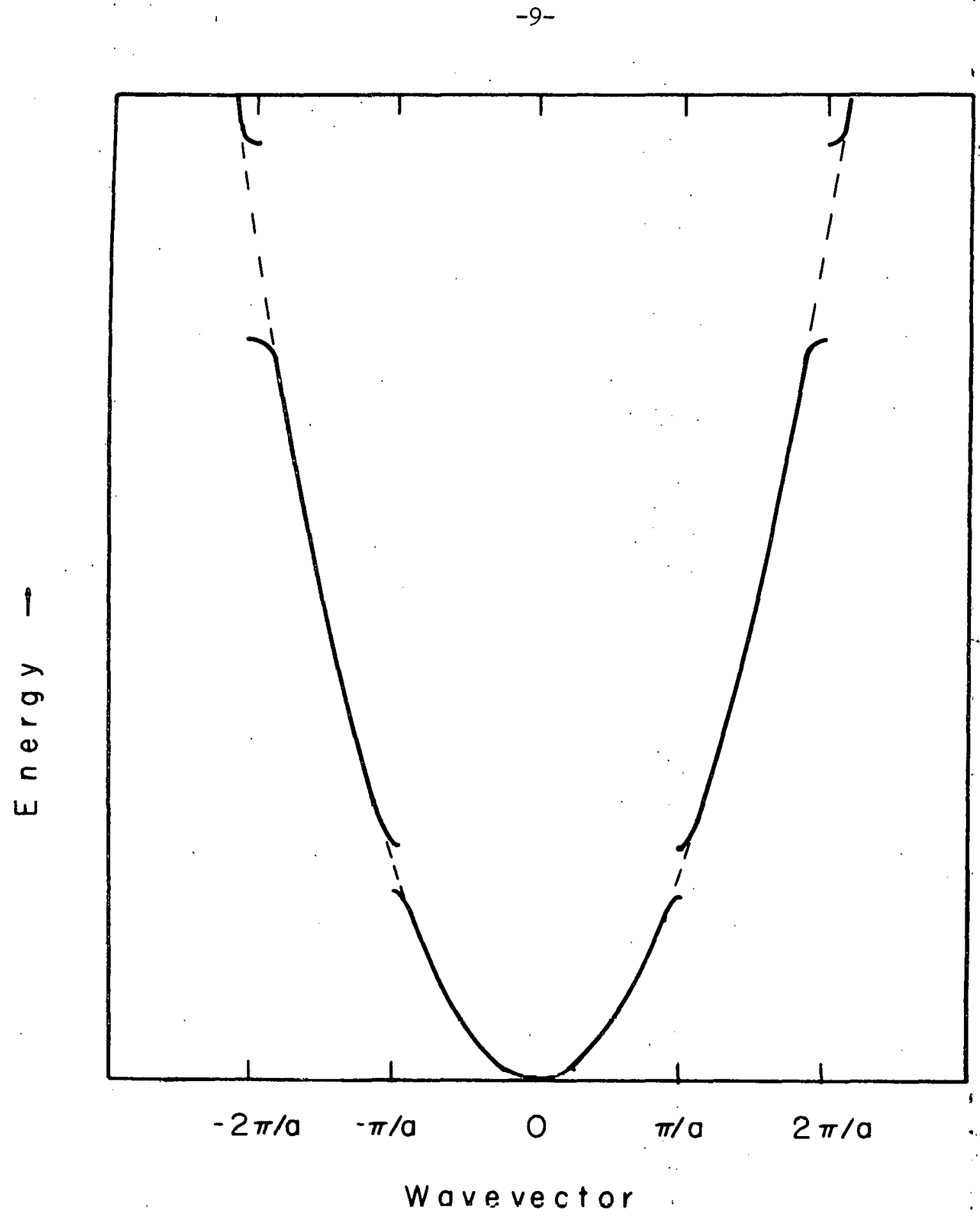

XBL $707-1510$

Fig. I. Nearly Free Electron Approximation - Extended Zones. 
can be generated with distinctly different energies but with $C_{0}^{2}=C_{m}^{2}$. It is apparent that one of the wavefunctions should be labeled $\psi_{\overrightarrow{\mathrm{k}}_{\mathrm{O}}}$ and the other $\psi_{\overrightarrow{k_{m}}}$, but the labels cannot be unambiguously assigned to one wavefunction: or the other. In order to avoid this difficulty, and more serious difficulties which occur when $U(\vec{r})$ is not small, $\vec{k}$ is restricted to values within the Wigner-Seitz cell of the reciprocal lattice (the first Brilluon Zone). The different wavefunctions which can be constructed from the same set of wavevectors are labeled by different band numbers, 1 . Within a band, $1, \underset{\overrightarrow{\mathrm{k}}, 1}{\mathrm{E}_{1}}$ is a continuous function of $\vec{k}_{\circ}$ The example shown in Fig. $I$ is redrawn in Fig. 2 in this "reduced zone" scheme.

The perturbation of the free electron wavefunctions that is caused by the potential, $U(r)$, leads from an energy which is simply dependent on $k^{2}$ to an energy which depends in a complex way on $\vec{k}$. The resulting Fermi surface reflects the symmetry of the lattice.

In surmary, then, the wavefunctions of conduction electrons are well approximated by plane waves in the interatomic spaces. Over a large fraction of wavevector space the free electron theory gives a reasonably good approximation to the energy and momentum states of the conduction electrons. The free electron theory predicts a constant density of occupied states in momentum space up to the Fermi momentum, and zero above it. The Fermi momentum is given by $p_{f}=\pi\left(3 \pi^{2} N / v\right)^{1 / 3}$. $\mathrm{N} / \mathrm{V}$ is the concentration of conduction electrons in the metal. 


\section{Positron Annihilation}

If a positron is injected into a metal it eventually annihilates wilh an electron. This usually results in the production of two gamma rays, each with energy approximately equal to $\mathrm{mc}^{2}$, where $m=$ electron mass, $c=$ speed of light. By conservation of momentum, the sum of the momenta of the gamma rays which are emitted is equal to the momentum of the annihilating pair. It is the momentum of the pair of gamma rays, or rather one component of it, which is measured in the experiment. When the pair annihilates, there is a certain probability that they will have a total momentum $\vec{p}$. This probability may be represented as $\rho(\overrightarrow{\mathrm{p}})$. This will also be the probability that a pair of gamma rays from the annihilation event will have momentum $\vec{p}$.

One component of the momentum of the pair of annihilation photons is measured in the following manner. Two gamma ray detectors, which are shielded by narrow horizontal slits, are set at some vertical angle, $\theta$. The angle is defined by the two slits and the source. Since the gamma rays are known to have momenta of mc each, they must have a vcrtical component of $\mathrm{mc} \sin \theta$ in oraer for both gammas to be detected. $m c \sin \theta=m c \theta$ in the limit of $\operatorname{small} \theta$. Whenever gamma rays are simultaneously detected in both detectors, a count is recorded. After a length of time, the angle is changed and the counting is repeated.

The number (or intensity), $I\left(p_{z}\right)$, of events which are detected with the component of momentum $p_{z}$ is just

$$
\begin{aligned}
& I\left(p_{z}\right)=C_{I} \int_{-\infty}^{\infty} \int_{-\infty}^{\infty} \rho(\vec{p}) d p_{x} d p_{y} \\
& p_{z}=m c \theta
\end{aligned}
$$




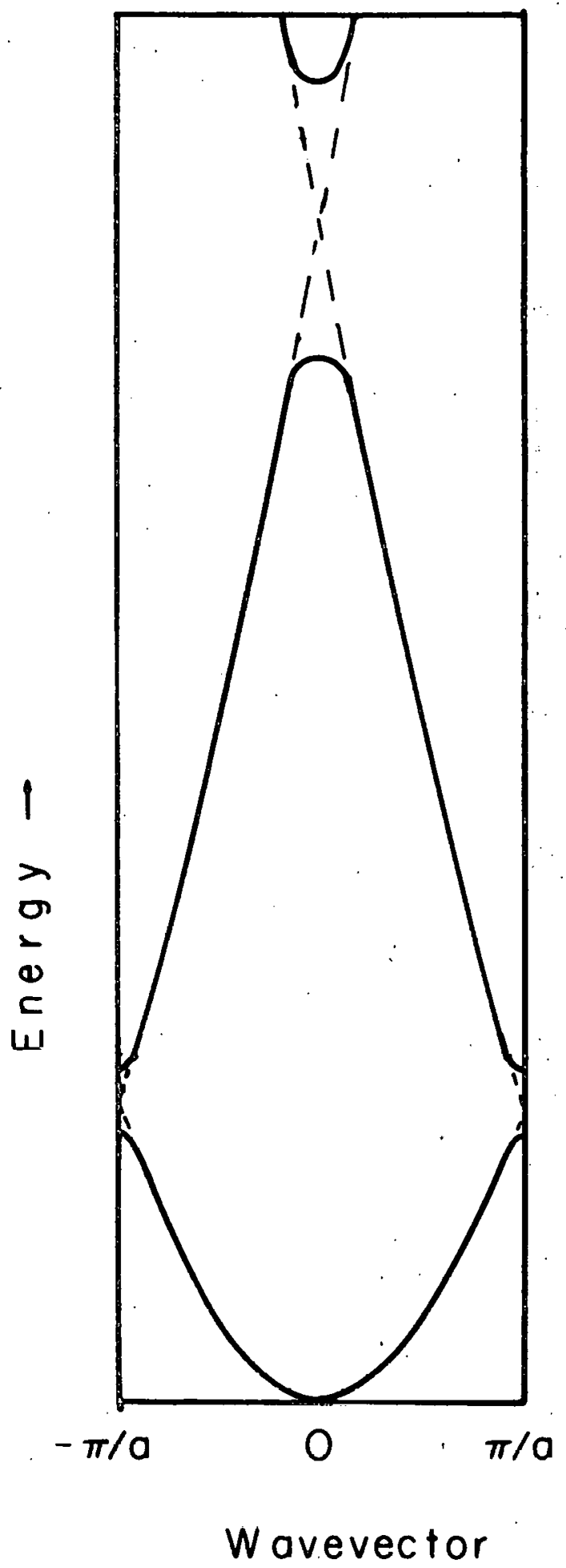

$X B \perp 707-1509^{\circ}$

lig. 2. Nearly Free Electron Approximalion - Reduced Zones. 
The first integration is performed because the detectors are insensitive to the slight shift in energy that is caused by a component of momentum toward or away from the detector. The second integration results because of the effectively infinite horizontal extent of the slits. If five conditions are met, the probability, $\rho(\vec{p})$, may be identified with the density of occupied electron states, $\omega(\vec{p})$. These conditions are:

1) That the positron penetrates to the bulk of the metal.

2) That the positron has no momentum of its own when it annihilates.

3). That the positron annihilates only with conduction electrons.

4) That the positron does not perturb the conduction electron states.

5) That the positron equally samples all the conduction electrons. These conditions will be discussed shortly, but first it is useful to calculate what the experimentally measured intensity curve will be if these conditions are met.

In the free electron approximation, $\underset{\overrightarrow{\mathrm{k}}}{\mathrm{E}}$ depends on $|\mathrm{k}|$, and not on $\vec{k}$. Thus a spherical Fermi surtace is predicted. The density of occupied states, $\omega(\vec{p})$, is a constant up to the Fermi momentum and zero thereafter. If the equation for $I\left(p_{z}\right)$ given above is expressed in spherical coordinates, it becomes after one integration with an isotropic $\rho(p)$,

$$
I\left(p_{z}\right)=c_{2} \int_{p_{z}}^{\infty} \rho(p) \text { pdp. }
$$

Since $\rho(p)=\omega(p)$, and $\omega(p)=$ Constant in the interral $0 \leq p \leq p_{f}$, and $\omega(p)=0$ if $p>p_{f}$, 


$$
\begin{aligned}
& I\left(p_{z}\right)=c_{2} \int_{p_{z}}^{p_{f}} c_{3} \text { pdp, } p_{2} \leq p_{f} \\
& I\left(p_{z}\right)=C_{4}\left(p_{f}^{2}-p_{z}^{2}\right), p_{z} \leq p_{f}, c_{4}=\frac{1}{2} c_{2} \cdot c_{3} \\
& \quad=0, p_{z}>p_{f} .
\end{aligned}
$$

The expected curve, then, is an inverted parabola which goes to zero at the Fermi momentum. 'This is 1llustrated in FIg. 3. Many metalo have exhibited this parabolic behavior. The Fermi momenta obtained by this method along with those predicted by the free electrun theory are shown in Table 1 . This tends to confirm the validity of the five assumplivins.

That the pnsitron will penetrate to the buik of the metal is easily demonstrated using the results of absorption experiments with positrons. These experiments indicate that the energetic pooitrons used in this experiment can easily penetrate several tenths of a millimeter into the sample. ${ }^{7}$ There is no doubt that the positrons penetrate to the bulk of the metal. There is almost a problem wilh lie pusitions pcnctrating too far. This will be discussed further in the experimental section concerned with the range of positrons.

That the positron will have no momentum of ils own when it annihilates is of course impossible. On the average it must have at Icast that amount of energy that is due to random thermld wotion. Gince this momentum would be small compared with the Fermi momentum, the problem. becomes one of showing that the positron is indeed thermalized before it annihilates. 


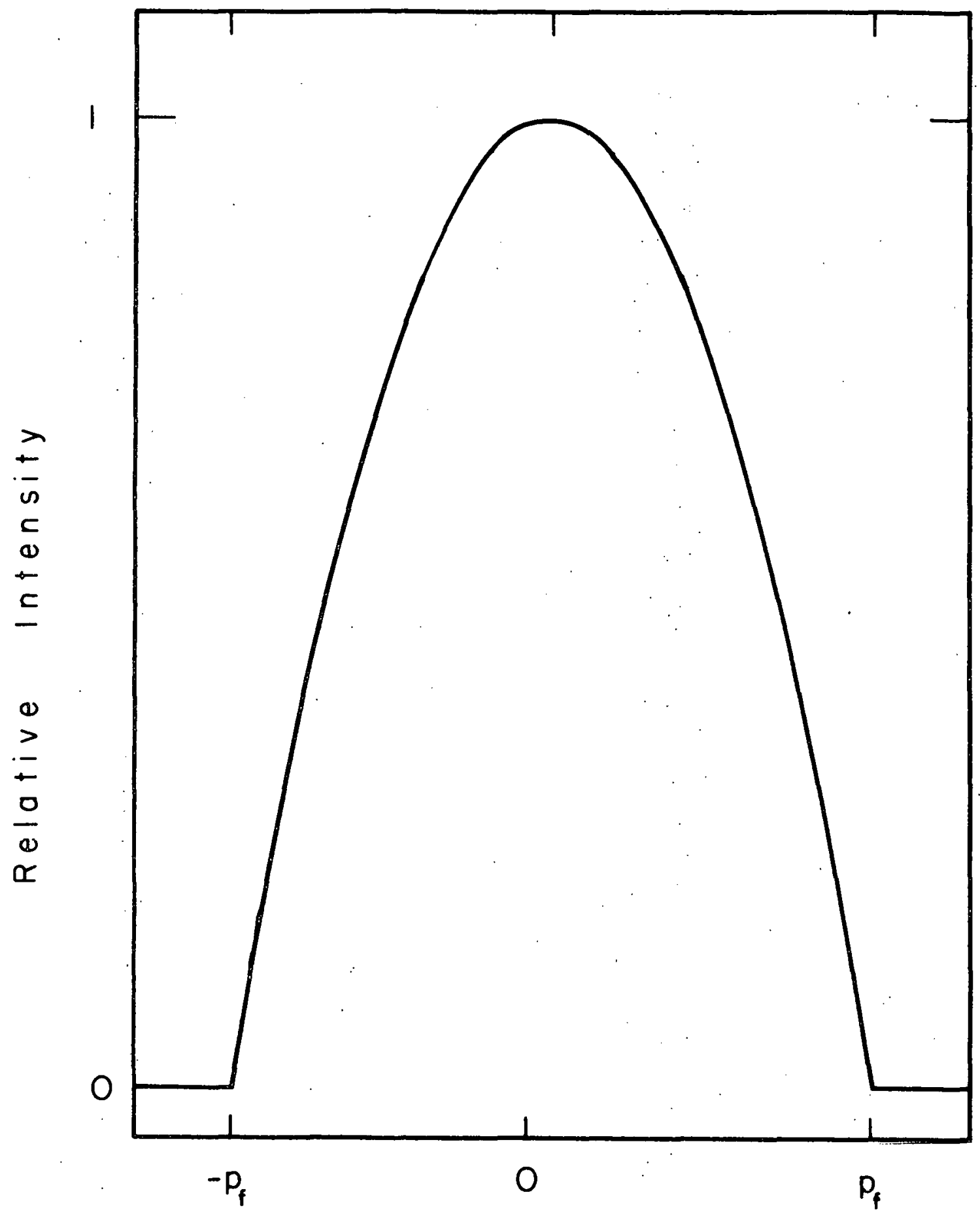

Momentum

Fig. 3. Theoretical Angular Correlation Curve. 
Table 1. Fermi momenta by positron annihilation

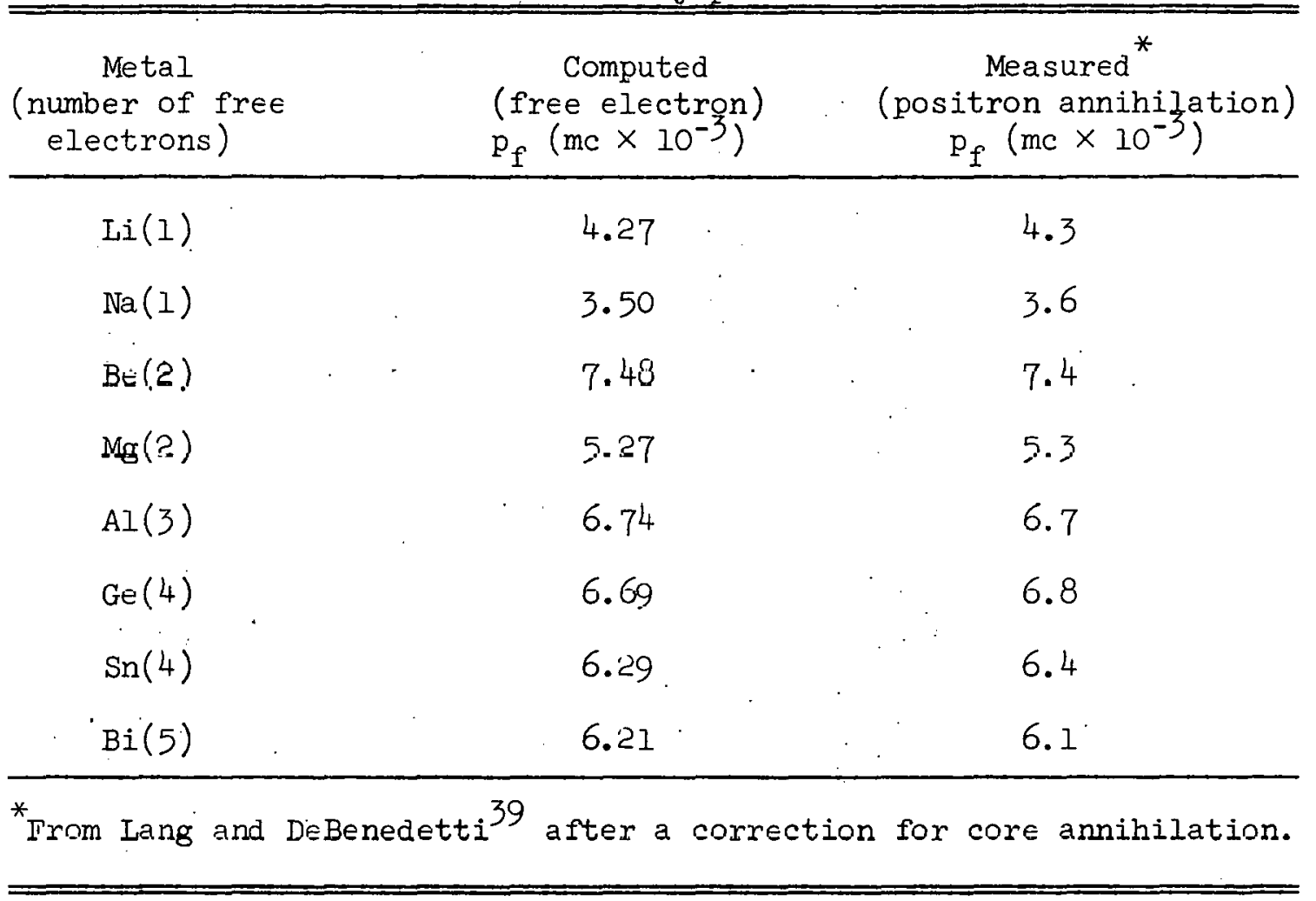


The thermalization of the positron is not difficult to understand since it is a charged particle and interacts strongly with the electrons and the lattice so that it quickly loses its excess energy. The probability that a positron will annihilate with an electron is small enough so that an energetic positron can scatter an electron without annihilating with it. An energetic positron can lose energy by scattering electrons to empty states in a higher energy band or to empty states within a band. It can also excite lattice vibrational modes. Early lifetime measurements by Bell and Graham ${ }^{8}$ indicated a lifetime for positrons in metals of about $1.5 \times 10^{-10} \mathrm{sec}$. The only calculation of thermalization time then available was by DeBenedetti et al. 9 It indicated a thermalization time of $3 \times 10^{-10} \mathrm{sec}$ in gold. Garwin ${ }^{10}$ pointed out that this calculated time was much too long because the calculation considered only the excitation of lattice vibrations (phonons) after the positron had reached an energy that was too low to allow it to cause interband transitions of electrons. It is by the excitation of interband transitions that the positron quickly loses the bulk of its energy. The calculation, then, was valid for insulators. In metals, however, the conduction electrons have available a near continuum of states so that it is possible for the positron to lose any amount of energy to the electrons. As again pointed out by Garwin, the positron cannot gain energy from the electrons since (at $0^{\circ} \mathrm{K}$ ) all lower energy states are already filled and any electron can only be scattered by the positron to an energy state above the Fermi energy, $E_{f^{*}}$ Thus it is possible for a positron with an energy of $0.1 \mathrm{eV}$ to lose energy to an electron at the Fermi surface which may have an energy of $5 \mathrm{eV}$. 
Garwin crudely calculated a thermalization time of about $10^{-14} \mathrm{sec}$. A more sophisticated calculation by Lee-Whiting ${ }^{\text {ll }}$ yielded the following times for energy loss: $4 \mathrm{eV}$ to $1 \mathrm{eV}$ in about $3 \times 10^{-15} \mathrm{sec}, 1 \mathrm{eV}$ to $0.1 \mathrm{eV}$ in about $2 \times 10^{-13} \mathrm{sec}, 0.1 \mathrm{eV}$ to $0.025 \mathrm{eV}$ in about $2 \times 10^{-12}$ sec. $0.025 \mathrm{eV}$ per particle corresponds to a temperature of $290^{\circ} \mathrm{K}$. It can be seen that with a lifetime of $1.5 \times 10^{-10} \mathrm{sec}$, the positron is almost certainly thermalized down to at least room temperature.

That the positron annihilates only with conduction electrons can be shown to be approximately true. The positron, bcing a positively charged particle, will have a very small probability of being near the. nucleus. Its probability amplitude will be large only in the interatomic spaces. In the independent particle approximation, the probability that an annihilating pair will have momentum $\vec{p}$ is propnrtional to

$$
\rho(\vec{p})=\sum_{k, l}^{\sum_{k}}\left|\int \exp (-i \vec{p} \cdot \vec{r} / r) \psi_{+}(\vec{r}) \psi_{\vec{k}, l}(\vec{x}) d^{3} \vec{r}\right|^{2} .
$$

where $\psi_{+}$is the positron wavefunction and $\psi_{\vec{k}, \ell}$ the electron wavefunction. $\mathrm{n}_{\overrightarrow{\mathrm{k}}, \ell}$ is the occupation number for the state $\overrightarrow{\mathrm{k}}, \ell \cdot \mathrm{n}_{\overrightarrow{\mathrm{k}}, \ell}=0$ ur 1. Since most core electrons have small probability of being at large distances from the nuclei, their contribution to $\rho(\vec{p})$ is small. The transition metals, with large numbers of d electrons which have it fáir probability of being at interatomic distances, notably do not show parabolic intensity curves.

'l'he last two conditions are best discusser tingether, sinoe thoy are part of the same theoretical problem. The positron is a positively charged particle, and its introduction into the metal must rcsult in sn 
increase in the density of highly mobile conduction electrons in its vicinity. That is, it must perturb the electron wavefunctions. This will, of course, lead to a considerable shortening of its lifetime in the metal. This is confirmed by comparing the observed lifetime of about $1.5 \times 10^{-10} \mathrm{sec}$ for metals with the value $7.5 \times 10^{-10} \mathrm{sec}$ which is obtained from theoretical estimates for aluminum which neglect coulomb attraction. 8 What effect this will have on the momentum of the annihilating pair is less obvious.

A large number of theoretical papers ${ }^{12-17}$ have been written in an attempt to find $\rho(\vec{p})$ for specific metals. The general results of these papers are:

(a) The concentration of electrons in the vicinity of the positron is much higher than in the unperturbed metal. This leads to a short half-life of the positron, in agreement with experiment.

(b) The momentum of the electrons in the vicinity of the positron is generally similar to the momentum distribution in the unperturbed metal, with a sharp break at the Fermi momentum.

(c) In the vicinity of the positron, thcrc are a few electrons in momentum states above what is the Fermi momentum in the unperturbed metal, even at $0^{\circ} \mathrm{K}$.

(d) The electron momentum distribution in the vicinity of the positron is slightly skewed such that the concentration of low momentum electrons is greatest.

(e) The probability that a positron will annihilate is greatest with electrons of the highest momentum. These last two effects tend to cancel oul and glve an observed annihilation rate as a f'unction of 
momentum which is remarkably similar to the momentum distribution in the metal in the absence of the positron.

(f) Although theory gives a very good fit to the low momentum part of the curve, it has been singularly unable to accurately reproduce the tail that is always observed at high momenta. This tail presumably is due to the positrons annihilating with core electrons。

Theoretical calculations, then, confirm that the angular correlation measurements can indeed be used to measure the general size and, if. single crystals are used, shape of the $\bar{F} \in$ rmi surf'ace. 


\section{MATHEMATICAL METHODS}

1. $\rho(\vec{p})$ and $n(\vec{p})$ from $I\left(p_{z}\right)$

The measured intensity curve for this experiment is given by the expression

$$
I\left(p_{z}\right)=c \int_{-\infty}^{\infty} \int_{-\infty}^{\infty} \rho(p) d p_{x} d p_{y}
$$

It is desired to extract the quantity $\rho(\vec{p})$ from the measured $I\left(p_{z}\right)$. This cannot be done analytically unless $\rho(\vec{p})$ is isotropic. That is $\rho(\vec{p})=\rho(p)$. In the experiments which were performed polycrystalline samples were used. The use of polycrystalline material insures that $\rho(\vec{p})$ will be isotropic. Using an isotropic $\rho(\vec{p})$ the integral, when expressed in spherical coordinates, becomes,

$$
I\left(p_{z}\right)=2 \pi C_{I} \int_{p_{z}}^{\infty} \rho(p) p d p
$$

If this is differentiated with respect to $\mathrm{p}_{\mathrm{z}}$, the expression

$\frac{d I\left(p_{z}\right)}{d p_{z}}=2 \pi C_{I}\left[\left(p \rho(p) \frac{d p}{d p_{z}}\right)^{p=\infty}-\left(p \rho(p) \frac{d p}{d p_{z}}\right)^{p=p_{z}}+\int_{p_{z}}^{\infty}\left(p \frac{d \rho(p)}{d p_{z}}+\rho(p) \frac{d p}{d p_{z}}\right) d p\right]$ is obtained.

or

$$
\frac{d \cdot I\left(p_{z}\right)}{d p_{z}}=-2 \pi c_{1} p_{z} \rho\left(\mu_{z}\right)
$$

$$
\rho\left(p_{z}\right)=-\frac{d I\left(p_{z}\right)}{d p_{z}} \cdot \frac{1}{2 \pi C_{2} p_{z}}
$$

the desired result. 
It is seen, then, that the density of states is simply related to the derivative of the experimental curve. It should be emphasized that this relationship is good only under the assumption that $\rho(p)$ is isotropic. This in general means that a polycrystalline sample must be used. If the sample used is a single crystal, it is necessary to use a trial $\rho(\vec{p})$ to calculate $I\left(\vec{p}_{z}\right)$. In the case of a single crystal, $\rho(\vec{p})$ cannot bo determined unambiguously unless the positron annihilation spectrum is measured in at least three different crystal directions.

In order to calculate $\rho\left(\mathrm{p}_{\mathbf{z}}\right)$ to within a cunstant factor, two quantities must be known; $d I\left(p_{z}\right) / d p_{z}$, and $p_{z^{*}}$. The slope, $d I\left(p_{z}\right) / d p_{z}$, can be calculated from the data. If the slopes are approximated by the ordinary method of calculating $\left(I_{2}-I_{1}\right) /\left(\theta_{2}-\theta_{1}\right)$. for adjacent points, however, the error in the slope can be very great. inls is especially true if the two points are close together, or lf une of lle juints has a large error. This situation can be improved considerably if use is made of the fact that the intensity is a continuous furction of the angle. The following interpolation technique can then be used. A point $\left(I_{i}, \theta_{i}\right)$ is chosen. The "slopes", $\left(I_{n}-I_{i}\right) /\left(\theta_{n}-\theta_{i}\right)$ are calculated for all points $\left(I_{n}, \theta_{n}\right)$ within some interval of $\theta_{i},\left|\theta_{n}{ }^{-} \theta_{i}\right|<\Delta \theta_{\max ^{\circ}}$ A continuous smooth function of $\theta-\theta_{i}$ is then fit through the appropriately weighted "slopes". The value of this function at $\theta-\theta_{i}=0$ is taken to be the true slope of the experimental curve at $\theta_{i}$. This is done for all points $\theta_{i}$ except for the few points at the ends of the curve where this method no longer gives significantly more precise values than the ordinary method. 
A program was written to do this on the CDC 6600. The smooth curve which was used in the fitting was a polynomial in $\theta-\theta_{i}$. The degree of the polynomial which was used depended on the number of points which were being fit. In no case did the number of independent coefficients to be determined in the fitting exceed half the number of points being fit.

$\mathrm{p}_{z}$ must also be derived from the data. This is because $\mathrm{p}_{z}$ itself, or quantities directly related to it, are not measured. Quantities related to some $\Delta \theta=\Delta \mathrm{p}_{\mathrm{z}} / \mathrm{mc}$ relative to an arbitrary zero are the measured quantities. It is known, however, that $I(\theta)$ must be symmetrical with respect to zero angle. Since the location of angle zero is not known from any direct measurement, it is necessary to determine it from the data itself. This is done in the following manner. An approximate zero angle is initially chosen. The appropriately weighted intensity points are then fit with a polynomial in the square of the angle by the method of least squares. Fitting the measured intensity with a polynomial in the square of the angle insures that the function will be symmetric about zero angle. The zero angle is then changed and the fitting repeated until a minimum is found in the appropriately weighted sum of the squares of the deviations.

A program was written to do this on the CDC 6600. An eleventh degree polynomial in the square of the angle was used in the fitting. This is equivalent to fitting with a twenty-second degree polynomial in the angle itself, while constraining the coefficients of all odd powers to be zero. There are twelve independent coefficients to be used in this fit. The typical measured curve has fifty points. The 
coefficients are, therefore, sufficiently overdetermined so that a reasonable fit is generated in the main part of the curve. The polynomial does, however, give an unrealistic fit between the widely spaced far tail points of the curve.

Once $\rho(k)$ is known, it is a trivial manner to calculate the number of electrons with $k$ values between $k$ and $k+d k$. This is just the difference in volume between spheres of radius $k$ and $k+d k$ multiplied by the density of states, $p(\mathrm{k})$. Thus

$$
\begin{aligned}
n(k) d k & =\rho(k) \cdot\left(4 / 3 \pi(k+d k)^{3}-4 / 3 r k^{3}\right) \\
& =\rho(k) 4 / 3 \pi\left(k^{3}+3 k^{2} d k+3 k(d k)^{2}+(d k)^{3}-k^{3}\right)
\end{aligned}
$$

dropping second and higher order terms in $\mathrm{dk}$

$$
\begin{aligned}
& n(k) d k=\rho(k) 4 \pi k^{2} d k \\
& n(k)=4 \pi k^{2} \rho(k) \\
& \text { 2. Effect of Temperature }
\end{aligned}
$$

The expected theoretical surve; which is a parabola with a sharp cut off, will be modified by the finite resolution of the instrument. This curve may also be slightly modified by the population of higher momentum states at t'inite temperature. Th1s slight blurring of the Fermi surface is described by the Fermic-Dirac distribution function.

$$
f(E)=\frac{1}{\exp \left[(E-\mu) / k_{\hat{B}} T\right]+1}
$$

$\mathrm{k}_{\mathrm{B}}=$ Bol.tzman constant; $\mathrm{T}$ = tcmperature (absolute).

The chemical potential (sometimcs aloo called the Fermi level.), $H$, is a function of temperature. At $0^{\circ} \mathrm{K}, \mu=\mathrm{E}_{\mathrm{f}}$. At temperatures small. compared with the Fermi temperature, $T_{f}=E_{f} / k_{B}, \mu$ is about equal to the Fermi energy. Since $\mathrm{T}_{f}$ is typically 50,000 : $\mathrm{K}$ for metals, $\mu$ 
is closely equal to $\mathrm{E}_{\mathrm{f}}$ at room temperature.

Assuming $\mu=E_{f}$ and including the Fermi-Dirac distribution, the following expression is obtained for the positron annihilation spectrum

$$
I\left(k_{z}\right)=C_{2} \int_{k_{z}}^{\infty} \frac{C_{3} k d k}{\left.\exp \left[k^{2}-k_{f}^{2}\right) k^{2} / 2 m k_{B} T\right]+I}, E_{k}=\hbar^{2} k^{2} / 2 m
$$

Making the substitution $x=k^{2}$, this is easily integrated to give

$$
\begin{gathered}
I\left(k_{z}\right)=C_{4}\left[x-\frac{\ln \left[\exp \left(C_{t} k_{f}^{2}\right)+\exp \left(C_{t} x\right)\right]}{C_{t}}\right]_{k_{z}}^{\infty}, C_{t}=k^{2} / 2 m k_{B} T, \\
C_{4}=\frac{1}{2} C_{2} C_{3} .
\end{gathered}
$$

Now

$$
\operatorname{limit}_{x \rightarrow \infty}\left[x-\frac{\ln \left[\exp \left(C_{t} k_{f}^{2}\right)+\exp \left(C_{t} x\right)\right]}{C_{t}}\right]=0
$$

So

$$
I\left(k_{z}\right)=c_{4}\left[\frac{\ln \left[\exp \left(c_{t} k_{f}^{2}\right)+\exp \left(c_{t} k_{z}^{2}\right)\right]}{C_{t}}-k_{z}^{2}\right]
$$

As is illustrated in Fig. 4, this thermal smearing of the Fermi surface is very small. It is in fact immeasurable with present techniques in positruin amihilation.

The momentum distribution of the thermalized positron is not negligible since its entire energy is its kinetic energy. Since only one positron at a time exists, in the sample, the Boltzman distribution may be uncd to give the average momentum of the positron. 


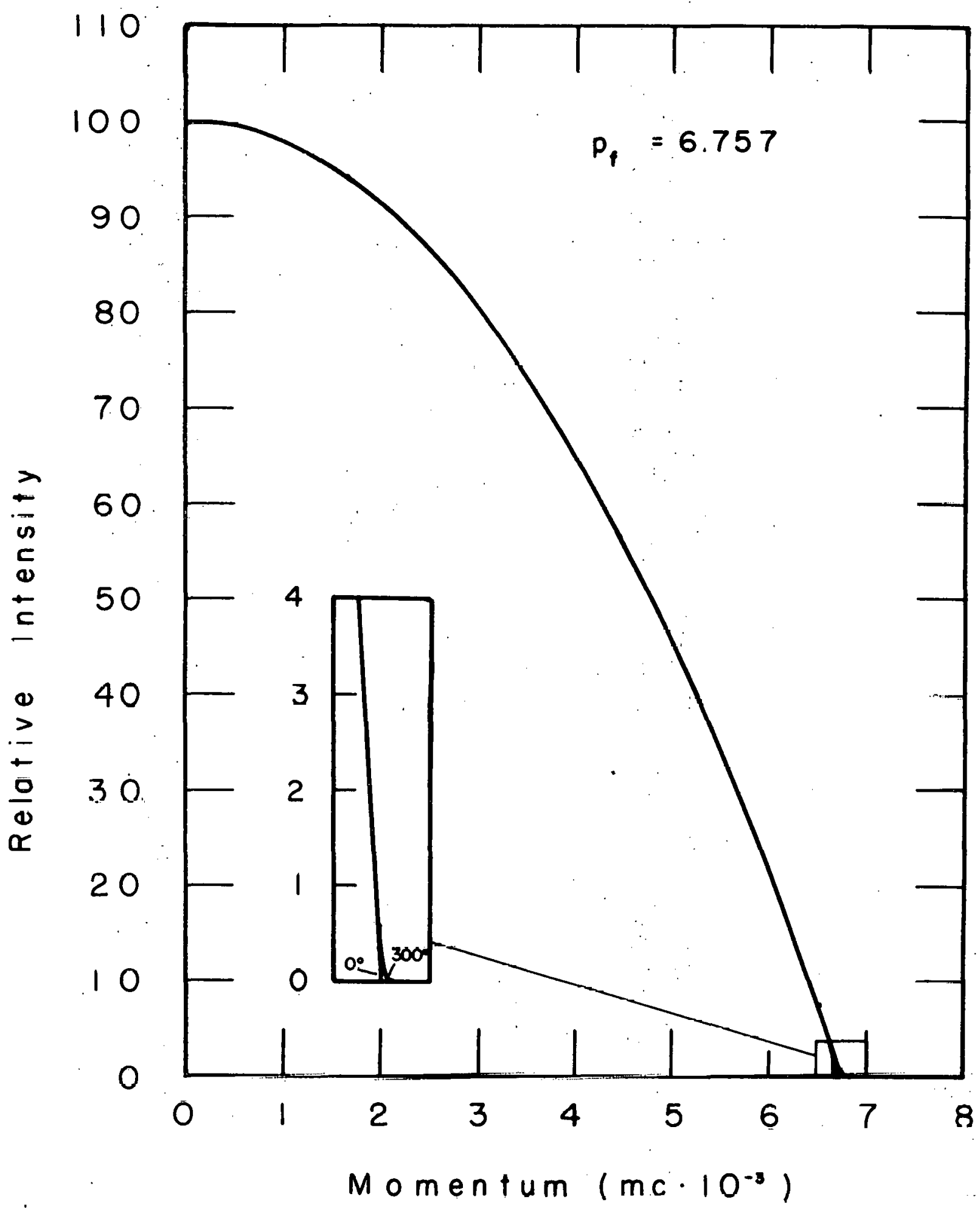

XBL $707-1508$

Fig. 4. H'flect of Therma.7. Smearing of the Fermi Surfacc on the Angular Correlation Curve. 


$$
\begin{aligned}
& \vec{p}_{+}=\left(\frac{8 k_{B} \operatorname{Tm}^{*}}{\pi}\right)^{1 / 2}, m^{*}=\text { effective mass of the positron } \\
& \vec{p}_{+} / p_{+}=\left(\frac{8 m^{*}}{\pi T m}\right)^{1 / 2}
\end{aligned}
$$

Assuming $\mathrm{m}^{*} / \mathrm{m}=1.9$ from stewart and Shand, 30

$$
\begin{aligned}
& \mathrm{T}=300^{\circ} \mathrm{K}, \mathrm{T}_{f}=50000^{\circ} \mathrm{K}, \\
& \overline{\mathrm{p}}_{+} / \mathrm{p}_{f}=0.17 \text {, an easily measured amount. }
\end{aligned}
$$

This smearing of the momentum distribution by the motion of the positron may be handled in the same manner as the effect of the finite angular resolution of the instrument. It is the same order of magnitude as the resolution of the instrument.

\section{Number of Electrons Annihilating}

In addition to the forementioned effects, there is almost always a tail to the inlensity curve extending to very large angles. This tail is presumably due to annihilation with core electrons, although it could also be due to annihilation with conduction electrons near the core where they have core-like wavefunctions. Whatever its origin, its contributicn to the slope of the intensity curve in the vicinity of zero angle appears to be small. This can be true even when its contribution to the intensity itself is large. If it is true that the contribution of this broad background to the slopes of the intensity. curve in the vicinity of zero angle is small, the slopes in the vicinity of zero angle should be characteristic of the conduction electrons. If the integral, $\int_{0}^{e s} r(k) d k, r(k) \propto k^{2} \rho(k)$, can be reasonably evaluated, 
it is possible to calculate the numbers of electrons annihilating at each pressure.

$I(k)$ is the measured intensity curve. Since $n(k)$ is proportional to $\mathrm{k} d \mathrm{I}(\mathrm{k}) / \mathrm{dk}$, it would seem to be a simple matter to evaluate the intergral $\int_{0}^{\infty} \mathrm{k} \frac{\mathrm{d} I(\mathrm{k})}{\mathrm{dk}} \mathrm{dk}$ either by some algebraic or graphical means. Unfortunately the data is not good enough to allow one to perform this integration with an uncertainty of less than 5 to $10 \%$. The integral $\int_{0}^{\infty} I(k) d k$ on the other hand, is easy to evaluate within a few tenths of a percent, either graphically or from the fitted curve. These integrals can both be expressed in terms of the density of states, $\rho(k)$, as

$$
\begin{aligned}
& \int_{0}^{\infty} n(k) d k=\int_{0}^{\infty} k^{2} \rho(k) d k, \text { and } \\
& \int_{0}^{\infty} T(k) d k=\int_{0}^{\infty} \int_{0}^{\infty} k \rho(k) d k d k .
\end{aligned}
$$

These two integrals appear to be similar; they can in fact be shown to be identical. If $\rho(k)$ is an isotropic, smooth, continuous function, as it is expected to be, it can be represented by a polynomial series, $\rho(k)=\sum_{n} C_{n} k^{n-1}$, in the interval $0 \leq k \leq k_{u}$. Since $\rho(k)$ rapidly approaches zero for large $k, k_{u}$ can be chosen to make $\rho\left(k_{u}\right)$ arbitrarily omall. Then, $\int_{0}^{\infty} k^{2} \rho(k) d k=\int_{0}^{k}\left(\sum_{n} e_{n} k^{n-1}\right) d k=\sum_{n} \int_{0}^{k} c_{n} k^{n-1} d k=\sum_{n} \frac{C_{n}}{n+2} k^{n+2}$. 


$$
\begin{aligned}
& \int_{0}^{\infty} \int_{0}^{\infty} k \rho(k) d k d k=\int_{0}^{k} \int_{0}^{k} u \sum_{n} c_{n} k^{n} d k \cdot d k \\
& =\int_{0}^{k} \sum_{n} \frac{c_{n}}{n+1}\left(k_{u}^{n+1}-k^{n+1}\right) d k \\
& =\sum_{n} \frac{C_{n}}{n+1}\left(k_{u}^{n+2}-\frac{k_{u}^{n+2}}{n+2}\right) \\
& =\sum_{n} \frac{C_{n}}{n+1} k_{u}^{n+2}\left(\frac{n+2-1}{n+2}\right) \\
& =\sum_{\dot{n}} \frac{C_{n}}{n+2} \cdot \dot{k}_{u}^{n+2} \\
& =\int_{0}^{\infty} k^{2} \rho(k) d k
\end{aligned}
$$

Q. E. D.

$I_{p}(k)$ is the quantity that is measured at pressure, p. The area of the measured curve is $A_{p}=f^{\infty} I_{p}(k) d k=c_{p} n_{p} n_{p}$ is the number of electrons which annihilate with the electrons. $c_{p}$ is a presently unknown factor to be found. If $I_{p}(k)$ is parabolic near zero angle,

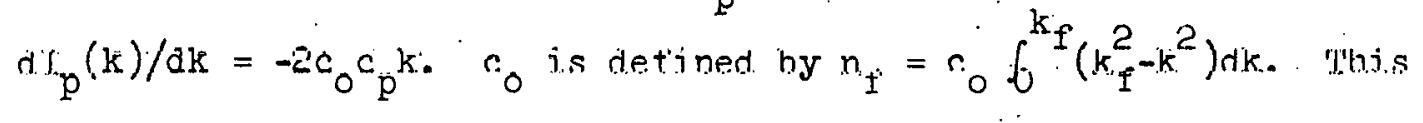


equation is derived from the free electron theory of metals. $n_{f}$ is the number of electrons which would give the material under the conditions at which $I_{p}$ was measured, a Fermi wavevector $k_{f} \cdot c_{0}$ is independent of the value of $n_{f}$ that is chosen, but it is a function of the volume of the metal. The value of $c_{p}$ may then be found from the experimentally measured $d I_{p}(k) / d k \cdot k$, and the theoretically derived $c_{0} \cdot n_{p}$ may be found from the equation $n_{p}=A_{p} / c_{p}$.

These methnds can nnly he used iff $I(A)$ is parabolic in tho vioinity of zero angle. If it is not, much of this information cannot be obtained. The relative widths of the measured intensity curves can be obtained, however, by the following method.

\section{Relative Widths of Curves}

If two intensity curves are fit with $\mathrm{m}-1$ degree polynomials,

$$
\begin{aligned}
& I_{1}(x)=\sum_{n=1}^{m} \cdot a_{n} x^{n-1} \\
& I_{2}(x)=\sum_{n=1}^{m} \quad b_{n} x^{n-1}
\end{aligned}
$$

the function

$$
F\left(x_{u}, C_{1}, C_{2}\right)=\int_{0}^{x_{u}}\left(I_{1}(x)-C_{2} I_{2}\left(C_{1} x\right)\right)^{2} d x
$$

describes the mean square deviation between the functions $I_{1}(x)$ and $\mathrm{C}_{2} \mathrm{I}_{2}\left(\mathrm{C}_{1} \mathrm{x}\right)$ in the inlerval $0 \leq x \leq_{4}^{\mathrm{x}}$. This w1ll be a minimum it' $\mathrm{C}_{1}$ is correctly chosen to reflect the difference in widths, and $\mathrm{C}_{2}$ is chosen to correctly adjust the relative intensities. By substituting the polynomial sums into the integral the following expression is obtained.

$$
F\left(x_{u}, c_{1}, c_{2}\right)=\int_{0}^{x_{u}}\left(\sum_{n=1}^{m}\left(a_{n}-C_{2} b_{n} C_{1}^{n-1}\right) x^{n-1}\right)^{2} d x .
$$


Let

$$
s_{n}=a_{n}-C_{2} b_{n} c_{1}^{n-1}
$$

Since

$$
\left(\sum_{n=1}^{m} s_{n} x^{n-1}\right)^{2}=\sum_{n=1}^{m} \sum_{j=1}^{m} s_{n} s_{j} x^{n+j-2}
$$

then

$$
\begin{aligned}
F\left(x_{u}, c_{1}, c_{2}\right) & =\int_{0}^{x_{u}}\left(\sum_{n=1}^{m} \sum_{j=1}^{m} s_{n} s_{j} x^{n+j-2}\right) d x \\
& =\sum_{n=1}^{m} \sum_{j=1}^{m} s_{n} s_{j} \frac{x_{u}^{n+j-1}}{n+j-1}
\end{aligned}
$$

where

$$
s_{n} s_{j}=a_{n} a_{j}-c_{2}\left(a_{j} b_{n} c_{1}^{n-1}+a_{n} b_{j} c_{1}^{d-1}\right)+c_{2}{ }^{2} b_{n} b_{j} c_{1}^{n+j-2}
$$

The function, $F$, will be a minimum for a given $x_{u}$ and $C_{2}$ when $0=\frac{\partial F}{\partial C_{1}}=\sum_{n=1}^{m} \sum_{j=1}^{m} \frac{x_{L}^{n+j-1}}{n+j-1}\left[-C_{2}\left(a_{j} b_{n} C_{1}^{n-2}(n-1)\right.\right.$

$$
\left.+a_{n} b_{j} c_{1}^{j-2}(j-1)+c_{2}^{2} b_{n} b_{j} c_{1}^{n+j-3}(n+j-2)\right]
$$

Similarly for fixed $x_{u}$ and $C_{1}$ 
$0=\frac{\partial}{\partial C_{2}}=\sum_{n=1}^{m} \sum_{j=1}^{m} \frac{x_{u}^{n+j-1}}{n+j-1}\left[-\left(a_{j} b_{n} c_{1}^{n-1}+a_{n} b_{j} c_{1}^{j-1}\right)+2 c_{2} b_{n} b_{j} c_{1}^{n+j-2}\right]$

or

$$
c_{2}=\frac{1}{\frac{m}{2}} \frac{\sum_{n=1}^{m} \sum_{j=1}^{m} \frac{x^{n+j-1}}{n+j-1}\left(a_{n} b_{j} c_{1}^{j-1}+a_{j} b_{n} c_{1}^{n-1}\right)}{\sum_{n=1}^{m} \sum_{j=1}^{m} \frac{x^{n+j-1}}{n+j-1}\left(b_{n} b_{j} c_{1}^{n+j-2}\right)}
$$

A program was written for the CDC 6600 to solve these equations for $C_{1}$ and $C_{2}$ by the method of successive approximations. The results, of course, are meaningfil only if $I_{1}$ and $I_{2}$ are similar in shape in the interval $0 \leq x \leq x_{u^{\circ}}$ 


\section{EXPERTMENTAL}

\section{High Pressure Apparatus}

The high pressure in these experiments was generated by the Bridgman opyosed anvil system. This system works in the following manrex. The sample to be pressurized is placed between the flat faces of two prestressed cemented tungsten carbide pieces. Surrounding the sample is a ring of pyrophyllite (a type of volcanic lava) coated with iron oxide. A uniaxial force applied along the axis of the anvils causes a quasi-hydrostatic pressure to be transmitted to the sample. The pyrophyl].ite ring acts as a gasket to keep the sample from extruding from between the anvil faces. Pyrophylite is chosen because of its unusual combination of high compressibility and large internal resistance to shear forces. It is coated with iron oxide to increase its coefficient of friction with the tungsten carbide anvils.

'the tungsten carbide inserts are prestressed in the following way. The tungsten carbide pieces and the hole in the retaining rings (or jackets) into which the tungsten carbide pieces are to he plared are machined with a taper of about $1^{\circ}$. The hole is made about 3 mil smaller than the insert. The insert is lubricated with a thin coat of Molykotc ${ }^{*}$ and forced into the jacket until it is flush with the top of the jacket. This requires about 75 tons of force. The anvil assembly is illustrated in cross section in Fig. 5. The sample assembly and pyrophyllite ring are shown in Fig. 6.

The metal discs which were used in the positron annihilation expterintuls were 7 mil thick and just under $5 / 16$ in. in diameter. The

\footnotetext{
Tradenark for finely divided molybdenum disulfide.
} 


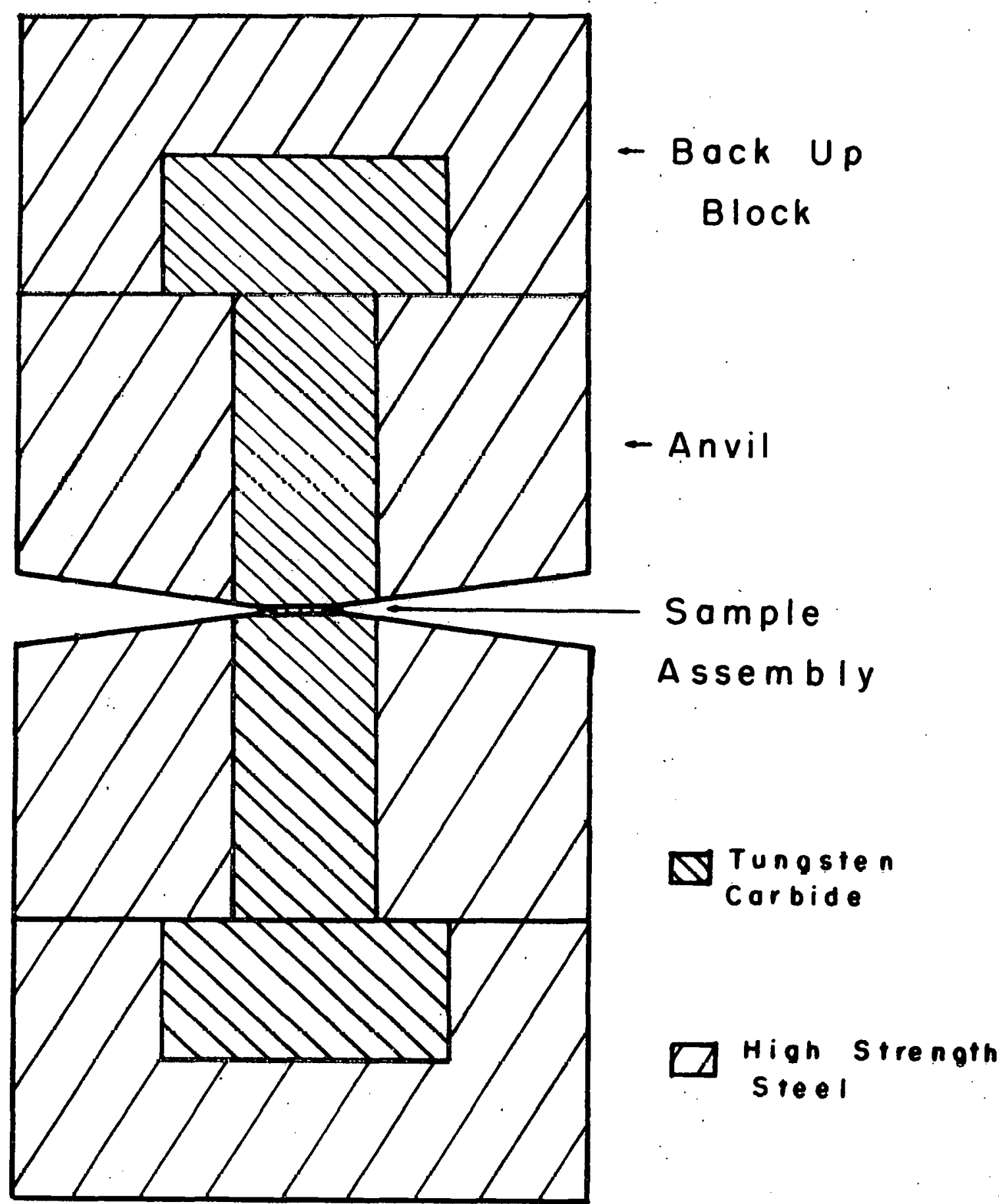

Fig. 5. Bridgman Anvil Assembly. 


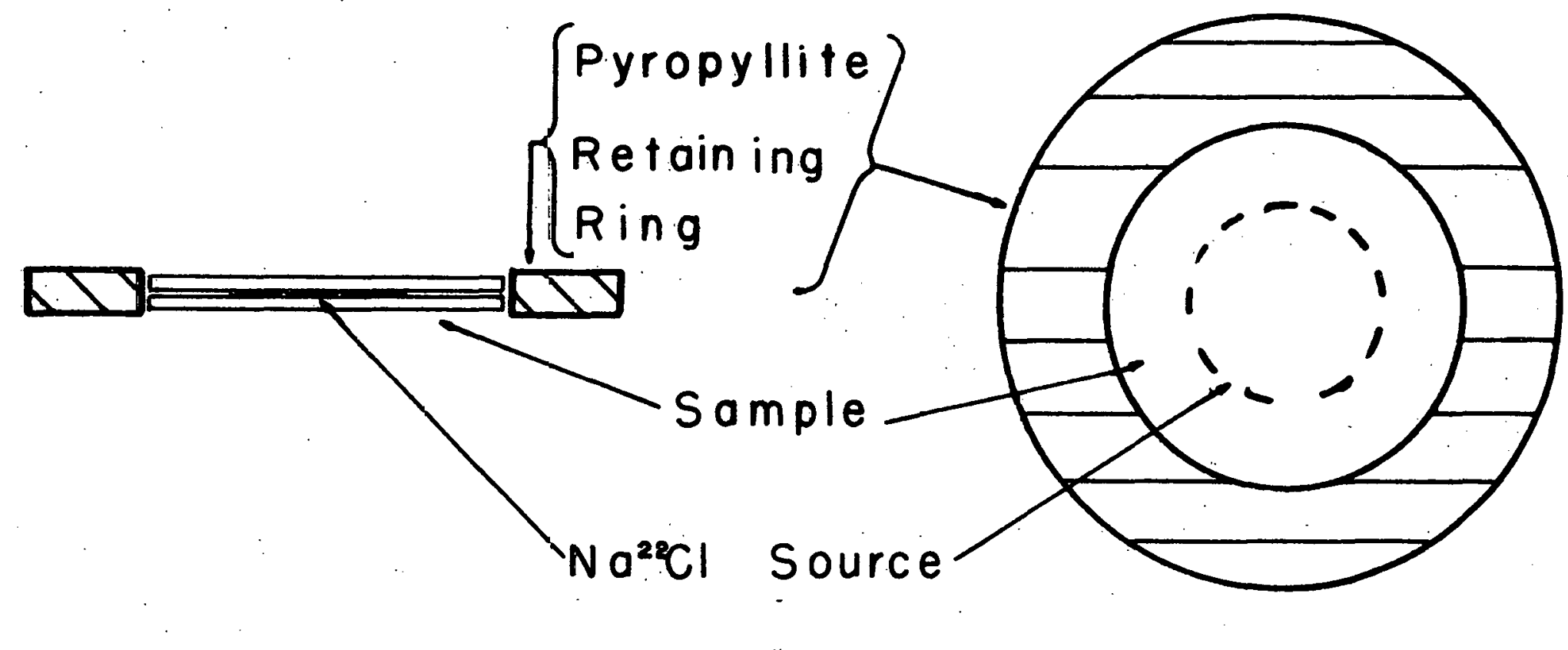

XBL 707-1505

Fig. 6. High Pressure Sample Assembly. 
pyrophyllite rings were $1 / 2$ in. o.d. $\times 5 / 16$ in. i.d. $\times 20$ mil thick. The flat faces of the anvils were $1 / 2 \mathrm{in.}$ in diameter. The sources in the various experiments varled from 1.0 to $1.5 \mathrm{mCi}$ of "carrier free" $\mathrm{Na}^{22} \mathrm{Cl}$. In the aluminum and bismuth experiments the $\mathrm{Na}^{22} \mathrm{Cl}$ was deposited directly on the metal discs by evaporation from a neutral aqueous solution. The source was then sealed by cementing the two discs together with epoxy. Because of the reactivity of the ytterbium metai, it was necessary to depusil lue $\mathrm{Na}^{22} \mathrm{Cl}$ on a picoo of $\mathrm{b} / 1 . \mathrm{mil}$ Mylar ${ }^{*}$ whirh was then placed between the discs of ytterbium metal. Tests mun a similar source indicated that the number of positrons anninilating in the source materlal is certainly less than $1 \%$ of the total number of positrons produced, and therefore entirely negligible for the purposes of this experiment.

The pressure was callbrated by using the Bismuth I-II, II-III, and III-V transitions, which are easily oeen as discontinuities in the resistance. I'he resistance of a bisiuth sample was mcacured as a func= tion of the force being applied to the anvils. The pressure is found to be a nearly linear function of this force. It was assumed that the three bismuth transitions occur at $25.4,27.0$, and 88 kbars. ${ }^{18-20}$ Due to unavoidable small differences in the geometry of the high pressure cel1, these points are not reproducible to better than $\pm 5 \%$. This lack of reproducibility is the limiting factor in determining the absolute pressure of the sample under investigation.

\footnotetext{
* Trademark
} 


\section{Range of Positrons}

When energetic positrons are injected into a solid they quickly lose their energy to the lattice by a large number of inelastic collisions with the electrons and the atomic cores. In addition to these collisions there are also a large number of elastic collisions which change the momentum vector but not the energy of the positron. All positrons must traverse approximately the same microscopic path length before being stopped in the material, but their macroscopic path lengths can be very different. These facts lead to an absorption law which is very different from the exponential law for gama or $\mathrm{x}$-rays. Some completely theoretical and some semi-empirical calculations have been reported. $21-23$ Ultimately the experimentally determined adsorption curves must be relied upon. $22-26$

For a monoenergetic beam of positrons approaching normal to the surface of the absorber, the absorption curve has the general. shape idealized in Fig. 7. This assumes that the transmitted positrons are measured in a $2 \pi$ geometry. The intial portion of the curve is concave toward the origin. This is followed by a long straight section which is usually cxtrapolated to an intersection with the abscissa. The absorber thickness defined by this intersection is called the practical range: The curve also exhibits a tail which goes to zero at a point called the absolute range.

Katz and Penfold ${ }^{7}$ have collected data from the literature. Using the data which they considered to be most reliable, they found the following empirical expression for the practical range of positrons in aluminum to be valid for initial positron energies, $E_{0}$, of from 0.01 to $2.5 \mathrm{MeV}$. 


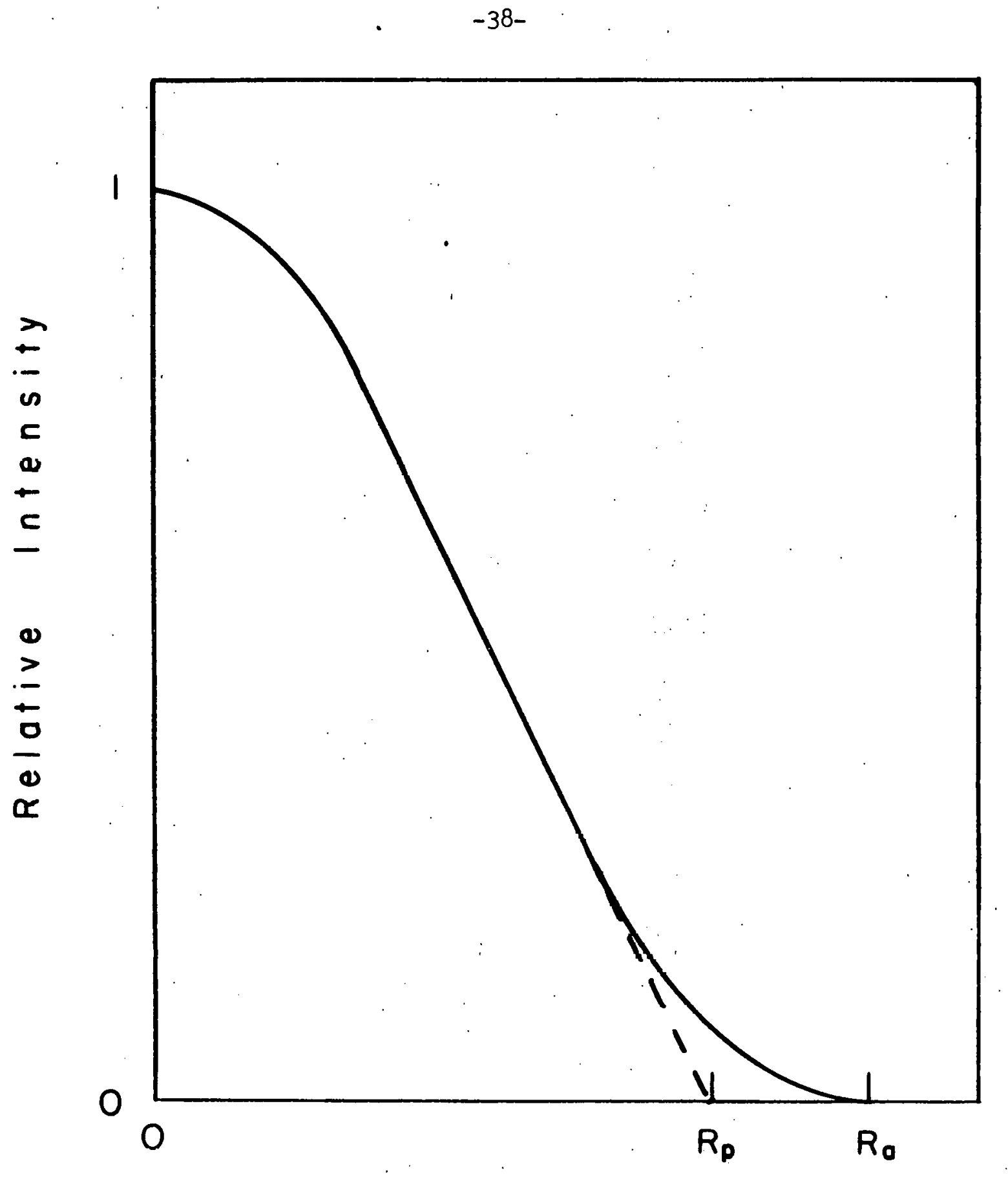

Absorber Thickness

XBL $707-1504^{\prime}$

Fig. 7. Typical Positron Transmission Curve. 


$$
R=412 \mathrm{E}_{\mathrm{O}}\left(1.265-0.0954 \ln \left(\mathrm{E}_{\mathrm{O}}\right)\right)\left(\mathrm{mg} / \mathrm{cm}^{2}\right)
$$

The $\mathrm{Na}^{22}$ source used in this experiment emits positrons with an initial energy of $0.54 \mathrm{MeV} .^{27-29}$ If this value is inserted into the proceeding equation, and if a density of $2.7 \mathrm{~g} / \mathrm{cm}^{3}$ is assumed for aluminum, the practical range which is obtained is $182 \mathrm{mg} / \mathrm{cm}^{2}$ or $26.5 \mathrm{mil}(0.674 \mathrm{~mm})$. This is much greater than the thickness of the sample used in this experiment, which is $7 \mathrm{mil}$. It is worthwhile to calculate how many positrons do penetrate the sample.

The probability that a positron will be emitted between an angle $\theta$ and $\theta+\mathrm{d} \theta$ from the normal to the surface of the sample is pnoportional to the solid angle which is subtended by these angles. The solid angle is by definition equal to the surface. area of a unit sphere subtended by the forementioned angles. The probability is then

$$
\begin{aligned}
P(\theta) \mathrm{d} \theta & =\mathrm{C}_{1} \int_{0}^{2 \pi} \int_{\theta}^{\theta+\mathrm{d} \theta} \mathrm{r}^{2} \sin \theta \mathrm{d} \theta \mathrm{d} \phi \quad r=1 \\
& =2 \pi \mathrm{C}_{1} \int_{\theta}^{\theta+\theta} \sin \theta \mathrm{d} \theta \\
& =2 \pi \mathrm{C}_{1}(\cos \theta-\cos (\theta+\theta)) \\
& =2 \pi \mathrm{C}_{1}(\cos \theta-(\cos \theta \cos (d \theta)-\sin \theta \sin (\mathrm{d} \theta)) .
\end{aligned}
$$

Expanding the functions of $d \theta$ in a power series and dropping second and higher order tenms in $\mathrm{d} \theta$,

$$
\mathrm{P}(\theta) \mathrm{d} \theta=2 \pi \mathrm{c}_{1} \sin \theta \mathrm{d} \theta .
$$


If $P(\theta)$ is to be normalized to unity in the interval $0 \leq \theta<\pi$,

$I=\int_{0}^{\pi} 2 \pi C_{1} \sin \theta d \theta$

$C_{1}=1 /(4 \pi)$

$P(\theta) \mathrm{d} \theta=\sin \theta \mathrm{d} \theta / 2$

In order to find the transmitted intensity, it is necessary to integrate the transmittance, $I(\dot{\prime})$, over $a \perp \perp$ ang $\perp$ s.

$I / I_{u}=\int_{0}^{\pi} T(\theta) P(\theta) d \theta$

If $\mathrm{D}$ is the thickness of the sample, the macroscopic path length is $\mathrm{D} / \cos \theta$. Assuming the transmittance to be a linear function of path length from $D$ to the practical range, $R$,

$T(\theta)=(R-D / \cos \theta)(T(O) /(R-D))$

$T(0)$ is the transmittance at $\theta=0$.

Assuming the transmittance to be zero for a path. lerigth greater than $R$, the integral becomes $\frac{1}{2} \int_{0}^{\theta} \max (R-D / \cos \theta) T(O) /(R-D)$ ained $\theta$

$\theta_{\max }$ is defined by $\mathrm{R}=\mathrm{D} / \cos \theta_{\max }, \theta_{\max }=\cos ^{-1}(\mathrm{D} / \mathrm{R})$.

The transmitted intensity is then,

$I / I_{0}=\frac{1}{2} \int_{0}^{\cos ^{-1}(D / R)}(R-D / \cos \theta) \frac{T(O)}{R-D} \quad \sin \theta d \theta=\frac{1}{2} T(0)\left(1+\frac{D \ln \left(\frac{D}{R}\right)}{R-D}\right)$

For $7 \mathrm{mil}$ aluminum, $\mathrm{D}=7, \mathrm{R}=26.5, \mathrm{~T}(0)=0.20$.

The $T(0)$ was obtained by interpolating from published data. 7,24,46 $I / I_{n}=0.24$

In this approximation some $24 \%$ of the positrons penetrate the aluminum sample. This approximation did not consider the tail of the transmittance curve which will increase this number, but it also did 
not consider the probability that a positron, after escaping the aluminum, will be scattered back into the aluminum by the tungsten carbide beneath it. These two effects should tend to compensate for each other. This answer may be considered to be essentially correct.

To be sure $24 \%$ is an unacceptably large number of events to occur in other than the desired sample material. Fortunately not nearly this number of undesirable effects are actually detected. If an event occurs in the aluminum, the pair of gamma rays must penetrate, on the average, about $7 \mathrm{~mm}$ of aluminum and $5 \mathrm{~mm}$ of pyrophyllite. If an event occurs in the tungsten carbide, it must penetrate about $1.2 \mathrm{~cm}$ of WC. Note that each individual gamma ray must penetrate only half this amount of material, but in order to be detected as an event both gammas must escape. The probability of this happening is the same as a single gamma ray of the same energy penetrating the total amount of material mentioned.

Pyrophyllite is a volcanic lava with the approximate chemical formuLa $\mathrm{Al}_{2} \mathrm{O}_{3} \cdot 4 \mathrm{SiO}_{2} \cdot \mathrm{H}_{2} \mathrm{O} \cdot 47$ The mass absorption coefficient of interest for $0.511 \mathrm{MeV}$ gamma rays are 48

$\begin{array}{ll}\text { Al } & 0.079 \\ \text { W } & (0.20) \\ \text { C } & 0.080 \\ \text { H } & 0.165 \\ \text { O } & (0.08) \\ \text { Si } & (0.08)\end{array}$

The quintities in parenthesis are interpolated from nearby elements. For $0.7 \mathrm{~cm}$ of aluminum, density $=2.7 \mathrm{~g} / \mathrm{cm}^{3}, I / I_{0}=0.86$. For $0.5 \mathrm{~cm}$ of pyrophyllite, average mass absorption coefficient 0.08 , denaity. $=2.9 \mathrm{~g} / \mathrm{cm}^{3}, I / I_{0}=0.89$ 
For $1.2 \mathrm{~cm}$ of WC, average mass absorption coefficient 0.19 , density = $11 \mathrm{~g} / \mathrm{cm}^{3}, I / I_{0}=0.082$.

The relative measured intensity is then

$$
\begin{aligned}
& \frac{I_{W C}}{I_{A_{1}}}=\frac{0.082}{0.86 \cdot 0.89} \times \frac{0.24}{1 .-0.24}=0.037 \\
& \frac{I_{W C}}{I_{A_{i}}+I_{W C}}=\frac{0.037}{1.037}=0.0 .36
\end{aligned}
$$

About $4 \%$ of the total detected coincidences come from the tungsten carbide anvils. For the $\mathrm{Yb}$ and $\mathrm{Bi}$ samples, the results are respectively about $1 \%$ and about $0.25 \%$ using mass absorption coefficients of 0.25 and 0.21 , and densities of 6.98 and 9.8 .

A $4 \%$ contribution to the total measured rate is not entillely negligible, but it should not seriously affect the results. The tungsten carbide has very low compressibility, therefore its positron annihilation spectrum should presumably be almost independent of pressure so that any change with pressure of the spectrum can clearly be ascribed to the aluminum sample. 


\section{Angular Correlation Apparatus}

The physical construction of the positron annihilation angular correlation apparatus is shown in Fig. 8. The scintillation counters are not shown in this figure. The apparatus was constructed to take advantage of the small vertical dimension of the high pressure sample in Bridgman anvils. The angular resolution of the instrument depends as much on the angular size of the sample viewed from the slits as it does on the angular size of the slits viewed from the source. The apparatus is simple in design, yet so conceived as to minimize alignment problems。 At the center of the apparatus is a sleeve into which the Bridgman anvils fit tightly. The sleeve is constructed such that when it is forced down onto the lower anvil until the inner ring in the sleeve rests on the lower anvil, the pivot on the outside of the sleeve is centered at the center of the top surface of the lower anvil. Both arms move on this pivot. The slits, which consist of two lead blocks each; spaced by shims of the desired thickness, are parallel to the bottom of their respective arms. The top surfaces of the lower lead blocks are at the same height from the bottom of their respective arms as is the center of the pivot. Thus, when assembled, the bottom surface of the slits are always pointed directly at the center top surface of the lower anvil. The 20 mil slits used in this experiment, therefore, point correctly at a 20 mil thick sample. Other sizes are in error by an insignificant amount.

In operation one arm is fixed at an angle approximately $90^{\circ}$ to the pressure transmitting column. The other arm is moved various amounts which are measrued by a dial indicator. The lndicator is fixed 

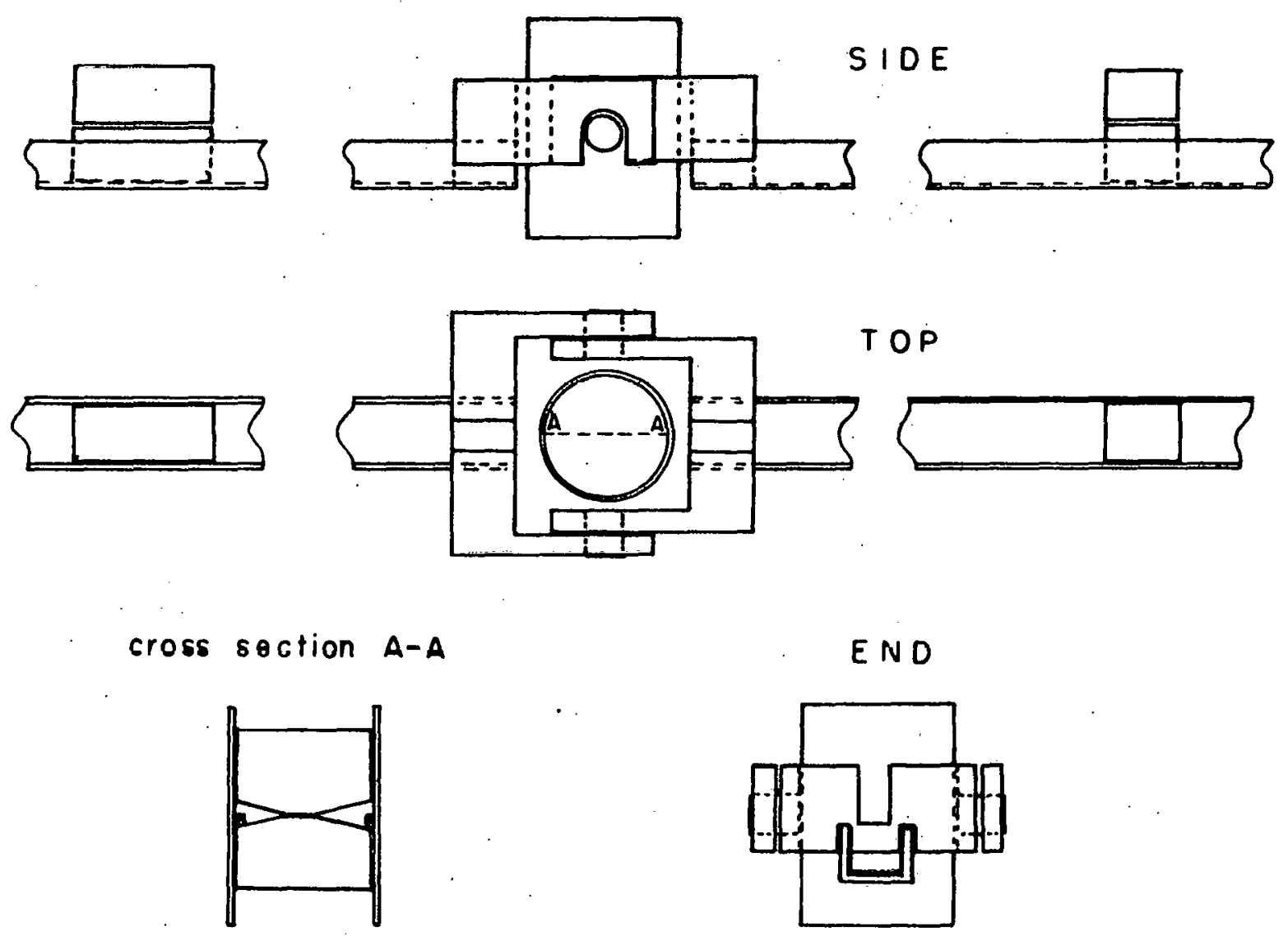

END

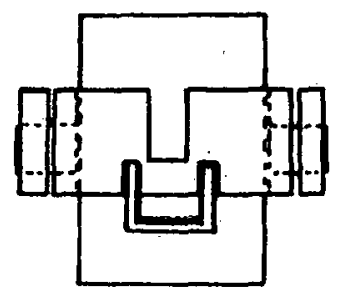

$\mathrm{XBL} 707 \cdot 1503$

Fig. 8. Angular Correlation Apparatus. 
perpendicular to the movabie arm 40.0 in. from the pivot and measures against a stationary surface. After counting an appropriate lenght of time, the counts from each detector and the coincidences are recorded.

The electronic circuit is shown in Fig. 9. The gamma ray detectors are $\mathrm{NaI}(\mathrm{TI})$-photomultiplier-preap assemblies. The NaI(TI) crystals are two inches in diameter and two inches long. The single channel analyzers are set to pass signal pulses corresponding to gamma rays of approximately 0.3 to $0.8 \mathrm{MeV}$ energy. This insures the inclusion of all $0.511 \mathrm{MeV}$ annihilation gammas while excluding the $1.3 \mathrm{MeV}$ gammas from the decay of $\mathrm{Na}^{22}$ to $\mathrm{Ne}^{22}$ and all low energy ganmas from scattering of the positrons and gamma rays.

The number of colncident counts and the total number of counts between 0.3 and $0.8 \mathrm{MeV}$ from each counter are collected in three different scalers. The three scalers and the elapsed time meter are controlled by a single mechanical switch which is operated by hand. The clock can be read to 0.01 minute and has at least this accuracy over a $12 \mathrm{hr}$ period. Since all points were taken with a running time of at least $50 \mathrm{~min}$, the errur in redalng the elapsed time is negligible.

All data were corrected for the small difference in counting rate at the movable arm as a function of angle. This difference is presumably due to the fact that at different angles the gamma rays must penetrate slightly amounts of mass. This correction is not large, amounting to in the most extreme case $3 \%$.

All data were corrected for random background coincidences. This correction is easily made since the number of random coincidences is proportional to the product of the total number of counts from each 


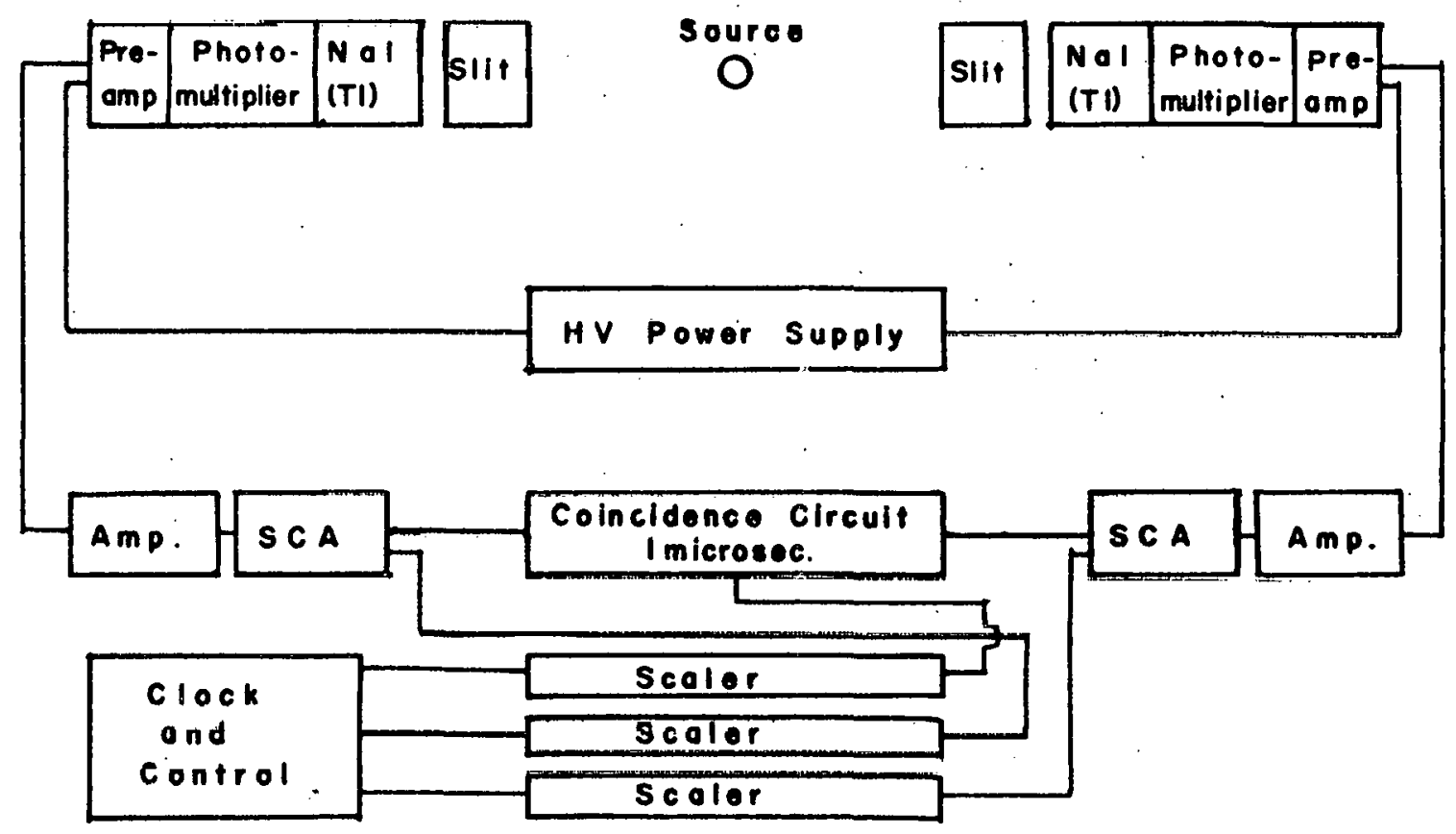

XDL 707-1499

Fig. 9. Electronic Block Diagram for the Angular Correlation Experiment. 


$$
-47-
$$

counter. The proportionality constant is easily found by measuring the number of coincidences with the apparatus set at a very large angle where all the coincidences are presumably random. 


\section{E. RESULTS AND DISCUSSION}

\section{Aluminum}

Two series of positron annihilation experiments were performed on aluminum as a function of pressure. The first series of experiments gave anomalous results. When the sample which was used in this first series of experiments was exposed, it was observed that at some time the pyrophylitite ring had ruptured and considerable extrusion of the aluminum had taken place; that is a "blow out" had occurred at some time during the scries of high pressure experiments. On the basis of the anomalous results it was judged that the "blow out" had occurred early in the series of experiments. This invalidated later results. The series of experiments was repeated with a new sample. This sample consisted ôt ábout $1 \mathrm{mCl}$ of "carrler free" $\mathrm{Nu}^{22} \mathrm{Cl}$ (from New Ingland Nuclear Corp.) between two discs of nominally $99.999 \%$ pure aluminum. These discs were $7 \mathrm{mil}$ thick and just under $5 / 16$ in. in diameter.

All data points were corrected for background counts, angle attenuation, and half life of the $\mathrm{Na}^{22}$ source. The points from a given presure, as explained earlier, were then fit with an eleventh degree polynomial in the square of the angle.

The fitted curve and data points in the primary part of the curve are shown in Fig. 10. These data were taken at 85 kbar. The error flags shown in the figure represent one standard deviation unit. The standard deviation relative to the measured intensity is equal to the reciprocal of the square root of the total number of counts which were accumulated at that angle. Data points from negative angles have been 
plotted as though they were taken at a positive angle; that is the curve has been folded over at zero angle. No error is shown in the measurement af the angle inoe: the angle measuring dev lce has marks at in-

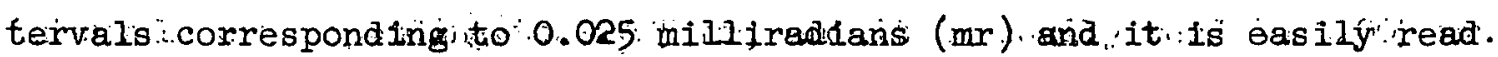

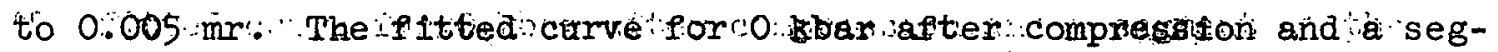

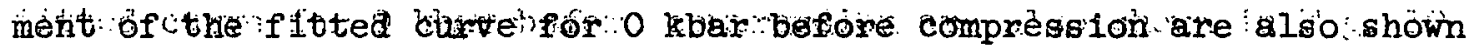

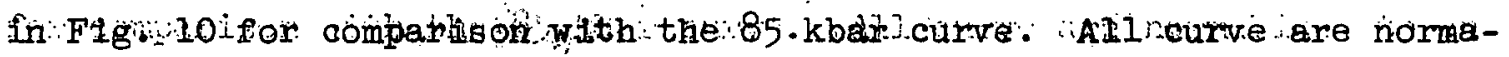
Iiżed

The slopes which were derived from the $85 \mathrm{kbar}$ Al data are shown in Fig. 11. The slopes. which were derived from points from the two different halves of the curve are shown with different symbols. As in. Fig. 10, all points are shown at positive angles only. All of the points shown have approximately the same absolute exror. The scatter of the points gives an estimation of this error. The standard deviation is estimated to be less than 0.01 in the units used; this is about $2 \%$ of the maximum slope. The solid curve is the analytical derivation of the curve which was fit to the original 85 kbar data points. The solid curve and the points were ohtained by essentially different methods, yet there is a remarkable degree of correlation between the two. This tends to confirm the validity of both methods of obtaining the derivative. The dashed curve in Fig. 1 la is the analytical derivative of the smooth curve which was fit to the zero kilobar points taken after compression to $85 \mathrm{kbar}$.

The densities of states which were derived from this slope data are shown in Fig. Ilb. The probable errors of the density of state points are very large at small angles and much smaller at large angles. In 


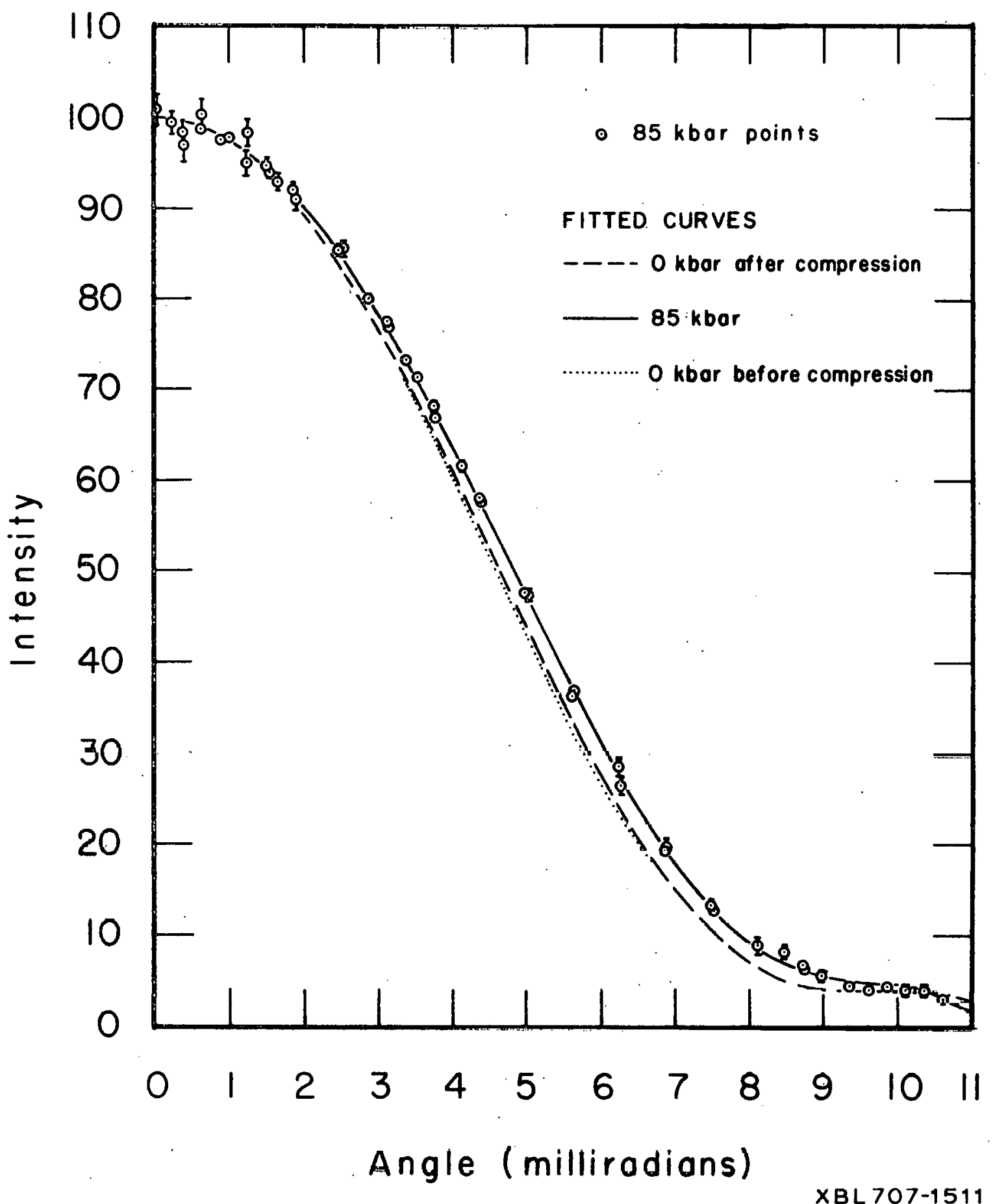

Fig. 10. Angular Correlation Curves from Aluminum. 


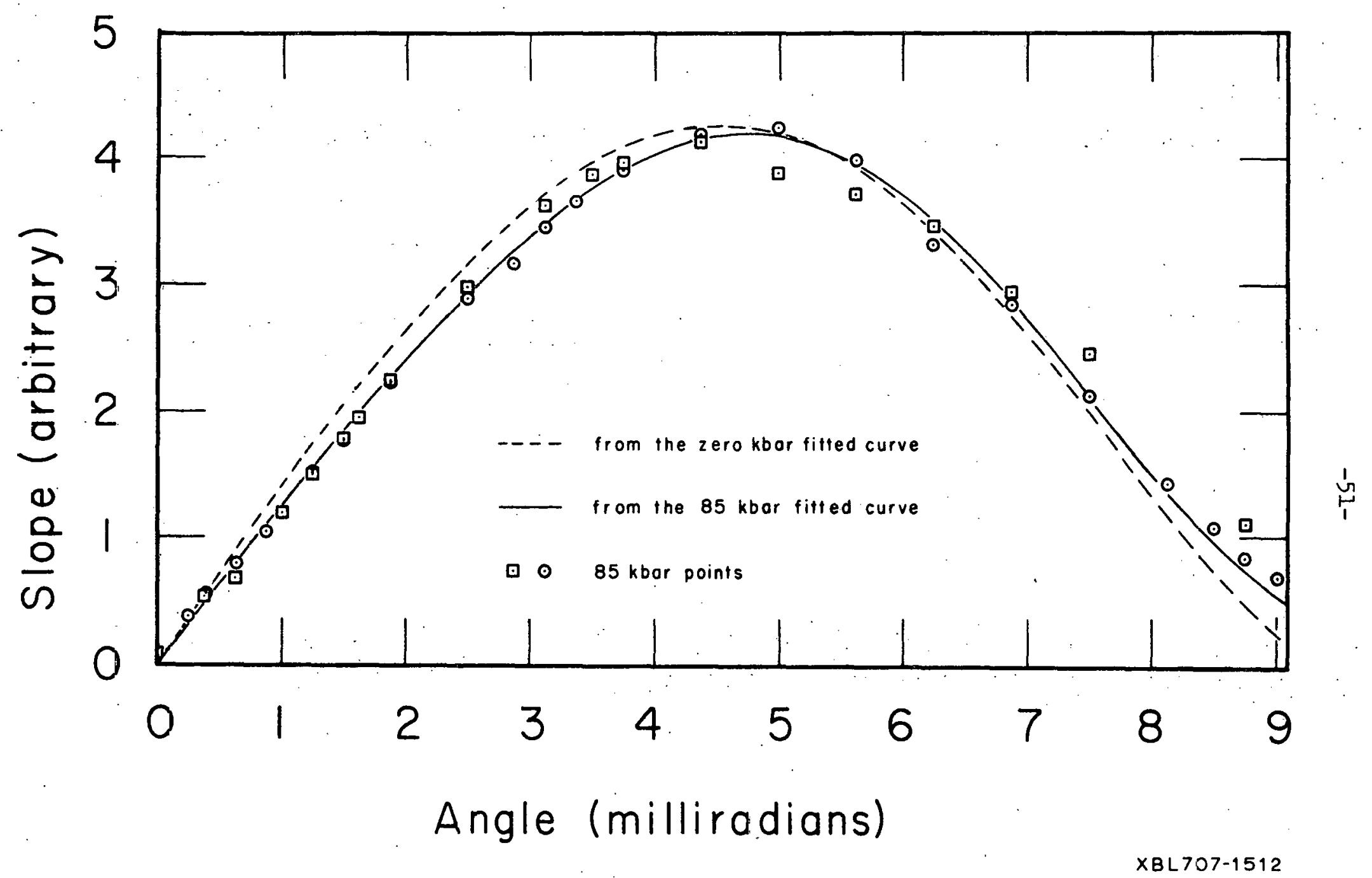

Fig. 1la. Slopes of the Aluminum Angular Correlation Curves. 


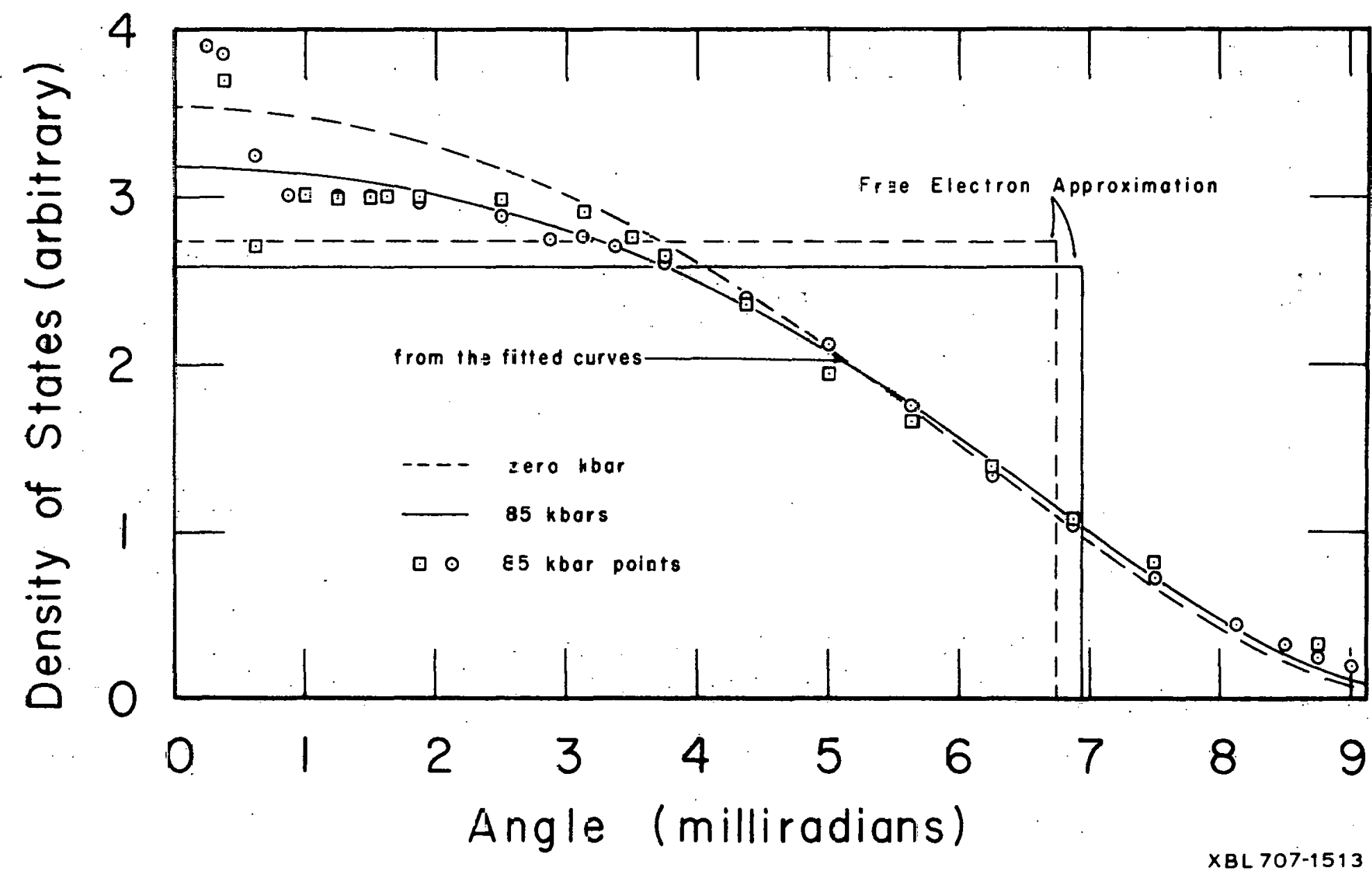

Fig. 1zb. Densitj of Occupiel States in Aluminum. 
the vicinity of small angles the smooth curve is probably more reliable than the points. Also shown in the figure are theoretical curves which are derived from a free electron model of aluminum at $0^{\circ} \mathrm{K}$. The theoretical angular correlation curve is assumed to have the same intensity at zero angle as the measured curve. This curves did not take into account the angular resolution of the apparatus. The theoretical Fermi momenta which are shown correspond to aluminum with densities of $2.70 \mathrm{~g} / \mathrm{cm}^{3}$ and $2.95 \mathrm{~g} / \mathrm{cm}^{3}$, that is aluminum at $0 \mathrm{kbar}$ and $85 \mathrm{kbar}$. The pressure-volume data which were used are those of Bridgman ${ }^{31}$ with corrections as suggested by Jamieson. 32

It is evident from ig. 1 lib that the positron annihilation curves which were measured for aluminum in this experiment do not reasonably approximate those predicted by the free electron theory. It is impossible to unambiguously assign a Fermi momentum to aluminum from this data. The Free Electron Theory predicts that the intensity curve will be parabolic in shape. A parabola was, therefore, Iit to the points. As points further from the center of the distribution were included in the fit, the width of the fitted parabola increased. Figure 12 illustrates this. If the points actually described a parabola, the width of the fitted parabola would not be a function of the number of points used in the fit. The expected width is that shown by the dashed line in Fig. 12.

The curve is narrower than expected. It seems possible that the strange shape of the aluminum intensity curve found here is due to the state of strain of the sample. I. Ya. Dekhtyar et al., 33 for example, found that the positron annihilation spectrum of aluminum narrows 


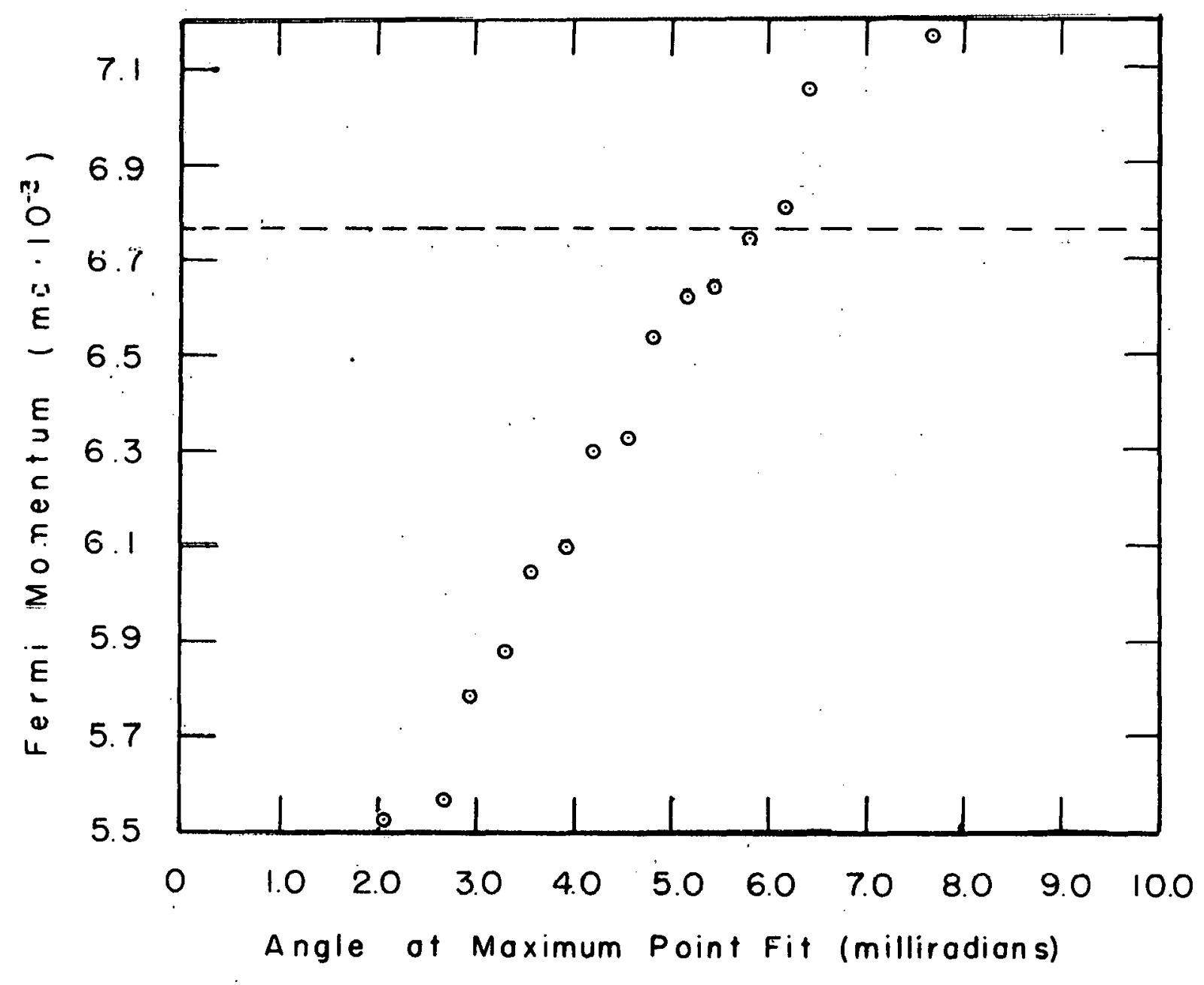

Fig. 12. Widthe of the Fitted Parabolas for Aluminum. 
considerably when the metal is plastically deformed. Kusmiss and Stevart ${ }^{34}$ found a similar effect for aluminum when it was heated. The change occurred well below the melting point, but no further change occurred upon melting. This would imply that the effect is related to the concentration of vacancies or other defects in the crystal. This also implies that the positrons may be attracted to and bound by vacancies or other defects. As this effect becomes better understood, perhaps it will become a tool in the study of these defects. In contrast to aluminum, Kusmiss and stewart ${ }^{34}$ found that bismuth showed this change only when actually melted, and sodium gave the expected parabolic shape even after it was melted. Mackenzie et al., 35 on the other hand, found no temperature effect for aluminum. The intensity curve they show for aluminum, however, does not appear to be parabolic. It also is narrower than would be expected for aluminum. That is, it is rather like the curve we measured. Chen et al. 86 also showed an aluminum intensity curve at room temperature and atmospheric pressure that is similar to ours.

It shomld he remarker in passing that we did get one and only one intensity curve for aluminum that was quite parabolic in shape and of the proper width for aluminum. This was the initial zero pressure run on the first aluminum sample: the one that blew out. This sample was not made of the $99.999 \%$ pure aluminum, but rather of $5 \mathrm{mil}$ and $1 \mathrm{mil}$ foil which was previously on hand and of uncertain purity.

All of the aluminum intensity curves taken from the second aluminum sample, however, are similar in shape. This makes it possible to compare their widths by the previously explained technique. The results 
of comparing the width of the curve taken at 0 kbar after compression with the widths of all the other fitted curves are shown in Fig. 13. The interval that was used was $0 \leq \theta \leq 6.75 \times 10^{-3}$ radians. The probable error in the width of the $85 \mathrm{kbar}$ curve was visually estimated. The errors at the other pressures were calculated from this value by assuming the probable error to be proportional to the reciprocal of the square root of the time spent in measuring the curve. The error bars on the 0 kilobar point after compression, which is the fiducial point, represent the probable error in the location of the starting point for the theoretical curve. The 0 kbar point after compression was chosen as the fiducial point because it was felt that this point is more representative of the state of strain of the sample than is the initial point. The theoretical curve is derived from the free electron theory which predicts that the Fermi momentum is inversely proportional to the volume of the sample to the one-third power. As before the pressurevolume data that were used were those of Bridgman with corrections as suggested by Jamieson.

It appears that the width of the measured curve scales up somewhat faster than $\mathrm{v}^{-1 / 3}$. This is surprising since it is expected that the width of the measured curve would scale up as $\mathrm{v}^{-1 / 3}$ even if the narrowing of the curve is due to positrons preferentially annihilating at vacancies. Calculation of the electron wavefunctions at a vacancy may, to a first approximation, be handled much like the particle in a box problem. This problem yields a $\mathrm{V}^{-1 / 3}$ dependence of the density of states. 


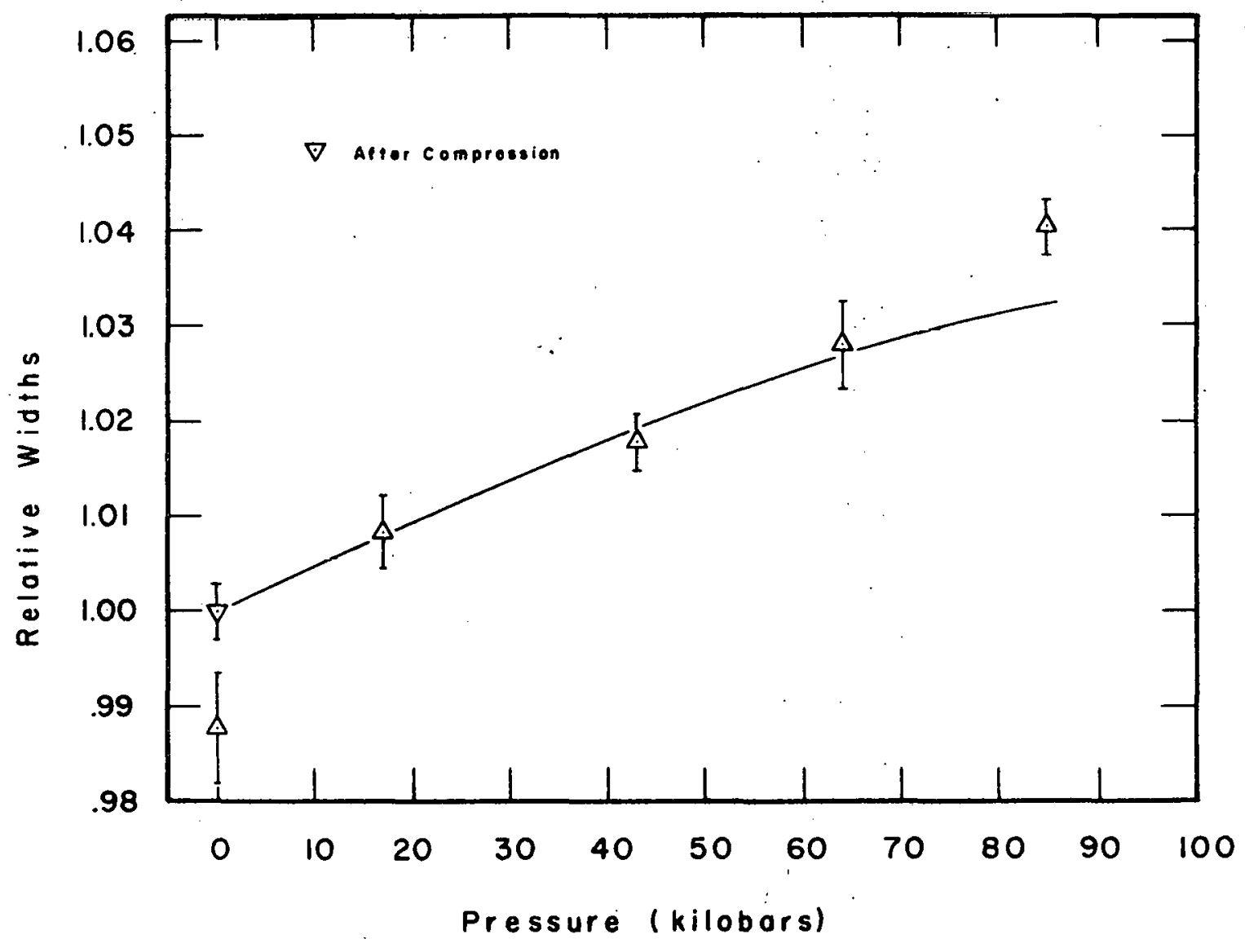

X日L $101-1447$

Fig. 13. Relative Widths of the Aluminum Curves. 
In addition to the departure from the $\mathrm{v}^{-1 / 3}$ behavior, there appears to be a permanent change in the width of the measured curve which persists at zero pressure. If a wide parabolic curve is associated with the contribution due to annihilation of the positron with ordinary conduction electrons, and if a narrower non-parabolic contribution is associated with the positrons annihilating with electrons at a vacancy, the following explanation can be offered for the deviation of the width of the curve from $\mathrm{V}^{-1 / 3}$ dependence. A lattice with a vacancy is larger than a perfectly ordered lattice with the same number of atoms by some amount, $\Delta \mathrm{v}$. If the pressure is increased an anount, $\mathrm{P}$, the internal energy of the lattice with a vacancy increases by an amount $P \Delta v$ relative to the perfectly ordered crystal. At very high pressures it will become favorable for vacancies to be destroyed. If this takes place in aluminum it would show up in this experiment as an anomalous increase in the width of the measured curvc. If oomc of the vacancias ware permanentiy destruyed, lhere wuld he a width imerease whieh pcroioto at zero pressure. This agrees with what was observed. The argument remains essentially the some if the defects causing the effect are not vacancies, but some other type of defects such as plane dislocations. It should be remarked that the expectation of a $\mathrm{V}^{-1 / 3}$ dependence of the width of the curve is an approximation only. Calculations suggest that it is not exactly the correct dependence for the Fermi momentum of aluminum. Asheroft ${ }^{37}$ has found that a reasonable fit with experiment is obtained for the electron wave functions of aluminum by using a weak pseudopotential approximation for an orthoganolized plane wave (OPW) calculation. A weak pseudopotential may be written in a 
Fourier series, $U(\vec{r})=\because \sum_{\mathbb{G}_{n}} U_{G_{n}} e^{-i \vec{G}_{n} \cdot \vec{r}}$, where $\vec{G}_{n}$ is a reciprocal lattice vector. Only two distinct Fourier coefficients are necessary to adequately describe the pseudopotential used in calculating the wavefunctions for the first three bands in aluminum. Ashcroft found that the values $U_{111}=0.0179$ Ry and $U_{200}=0.0562$ Ry give the best fit to existing de-Haas-van Alphen data. Mela $z^{38}$ has found the pressure derivatives of these coefficients from de-Haas-van Alphen effect studies to $7 \mathrm{kbar}$. They are $\mathrm{dU}_{111} / \mathrm{dP}=1.6 \times 10^{-4} \mathrm{Ry} / \mathrm{kbar}$, and $\mathrm{dU}_{200} / \mathrm{dP}=$ $2.1 \times 10^{-4}$ Ry/kbar. Assuming these derivatives to be constant to 100 kbar, Burton ${ }^{3}$ calculated an average momentum at the Fermi surface which was $0.04 \%$ larger than that expected from the free electron model at 100 kbar.

The extrapolation from $7 \mathrm{kbar}$ to $100 \mathrm{kbar}$ is a long one indeed. It is reasonable to expect that the first derivatives of the pseudopotential coefficients found by Melz will not be valid at. 100 kbar. It is not possible to predict in a straight-forward way from a-priori considerations how fast the potential will change with pressure. However, it is not unreasonable to expect that it may increase faster than linearly with pressure. This leads to an even greater departure from the $\mathrm{V}^{-1 / 3}$ dependence.

To the author's knowledge no one has performed a calcuilation on the expected angular correlation distribution of annihilation gammas from a positron bound to a vacancy in aluminum. It is not even certain that a positron can be bound by a vacancy in aluminum at room temperature or above. Until such a calculation is performed it cannot be said with certainty whether or not the strange angular correlation 
curves measured here are due to the postulated preferential positron annihilation at a vacancy.

\section{Bismuth}

The positron source for the bismuth experiment consisted of about $1.5 \mathrm{mCi}$ of "carrier free" $\mathrm{Na}^{22} \mathrm{Cl}$ from New England Nuclear Corp. This was evaporated onto a disc of bismuth metal 7 mil $(0.178 \mathrm{~mm})$ thick and just under $5 / 16$ in. $(0.794 \mathrm{~cm})$ in diameter. A second bismuth disc was epoxied to the first to form a sealed source. The discs were obtained by punching them from a 7 mil thick piece of bismuth foil. The foil was prepared by melting pellets of nominally $99.999 \%$ pure bismuth metal from Cominco American Inc. between two pieces of aluminum which had been milled flat. Seven mil shims were placed between the aluminum pieces on top of the two pieces to force the liquid bismuth to the proper thickness. Etching with nitric acid revealed that the foil was largely polycrystalline, as desired. The grains, however, were quite large, often with dimensions of a millimeter or more:

All data were corrected for background, angle attenuation, and half life of the $\mathrm{Na}^{22} \mathrm{Cl}$ source. The intensity curves for two pressures are shown in Figs. 14. All the points are shown at positive angles. The location of zero angle was found by the previously described technique of fitting the points with a polynomial. in the square of the angle. The slopes derived from the $0:$ kbar data by the previously described interpolation technique are shown in Fig. 15. This curve is typical of the bismuth curres. The slope appears to be linear with angle for at least the first $4 \mathrm{mr}$. If this is true the density of states should be a constant over that interval. The density of states 
$-61-$

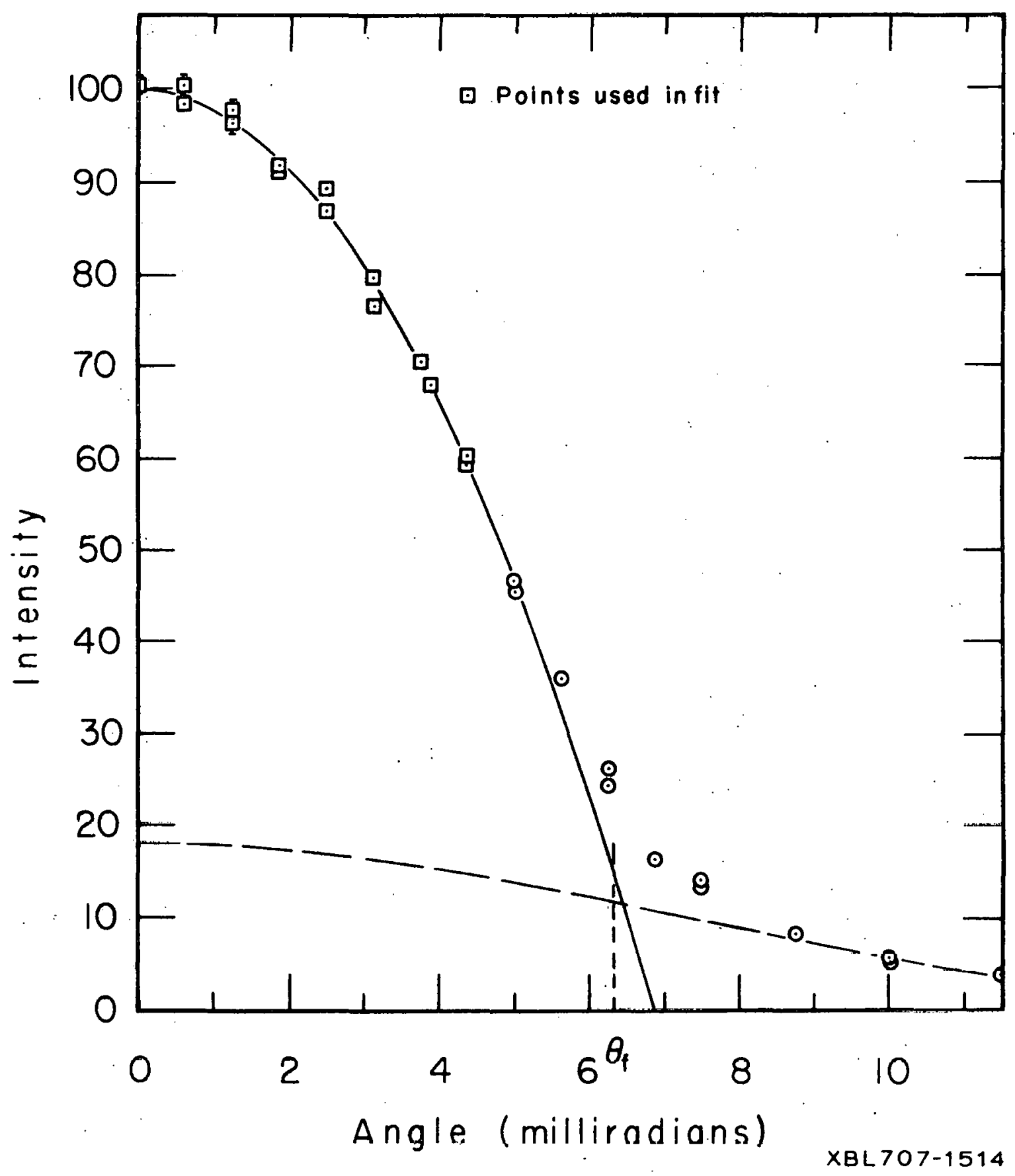

Fig. 14a. Angular Correlation Curve from Bismuth at 20 kilobars. 


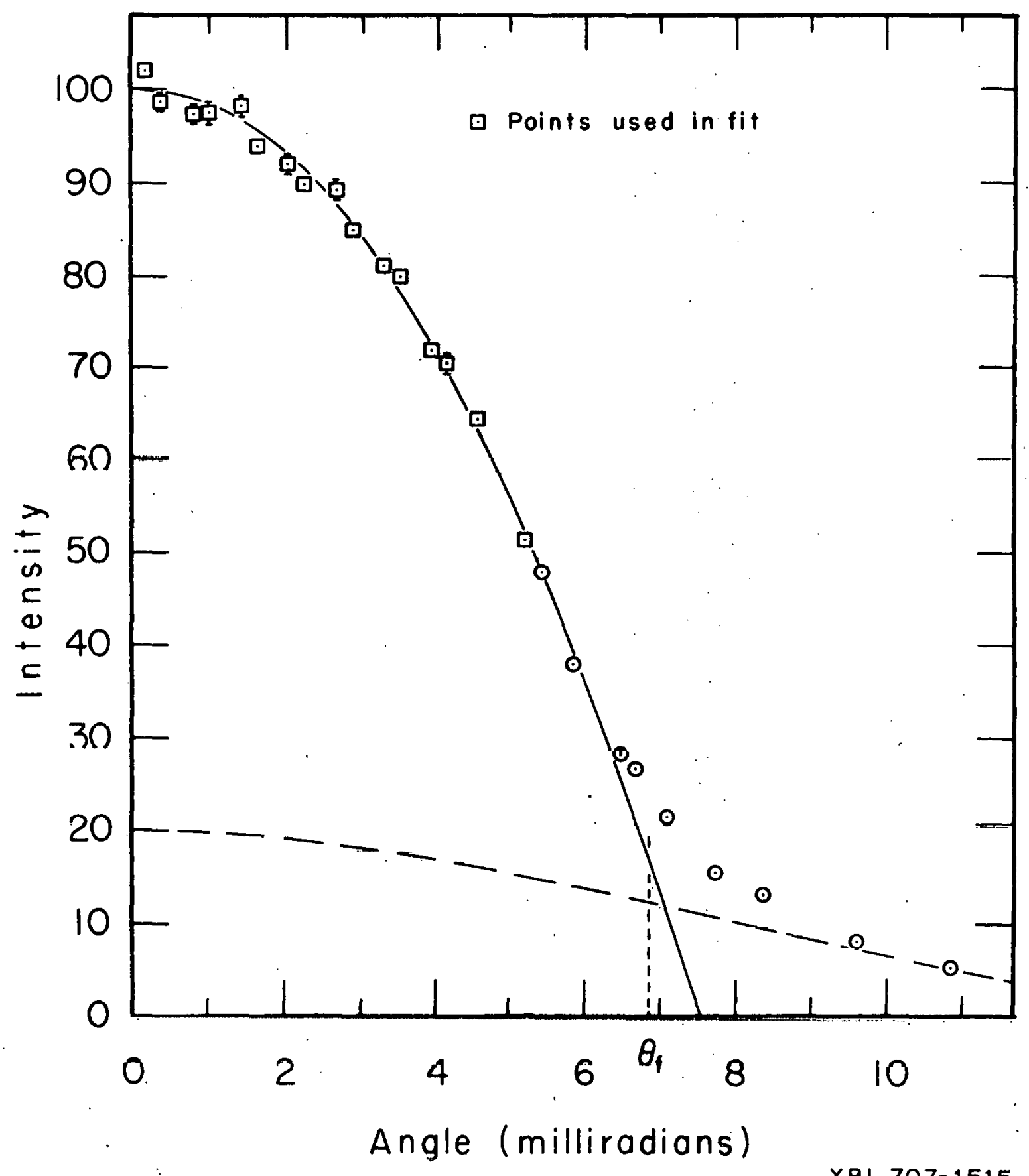

Fig. 1.4b. Angular Correlation Curve from Bismuth at 95 kilobars, 


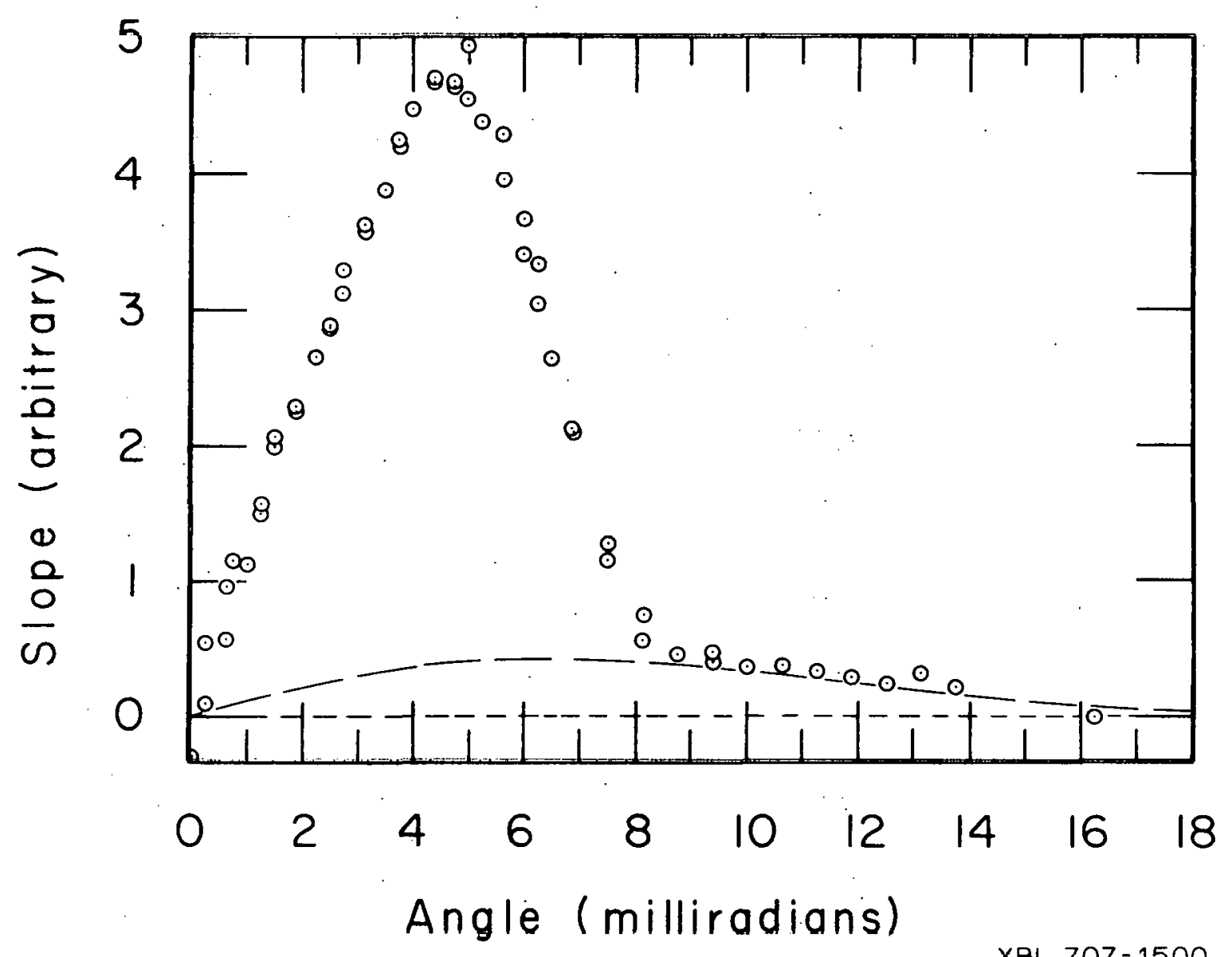

Fig. 15. Slopes of the 0 kilobar Bismuth Curve. 
for the 0 kbar experiment as a function of angle are shown in Fig. 16. Within experimental error, which is quite large at small angles, the density of states is a constant. This implies that the intensity curve is a parabola in that interval, and that a Fermi momentum can be derived from the data.

The data points were fit with parabolas. One parabola at each of the two pressures shown in Fig. 14 are shown along with the data points. The Fermi moment.a nhtained from tho fitted parabolos are slivin in Fig. 18. The error flags were estimated from the scatter of the Fermi momentum as different numbers of points were used for the fit. The solid line in the figure is the Fermi momenta predicted by the free electron theory. The free electron calculation assumed that bismuth has an initial density of $9.8 \mathrm{~g} / \mathrm{cm}^{3}$ and that Bridgman's compressibility data ${ }^{40}$ are correct. The B1 I-II and 11-1II transitions at 25.4 and $27.0 \mathrm{kbar}$ have been shown as if they were one. Bridgman's repurted volume discontinuities at 45 and $70 \mathrm{kbar}$ have been smoothed over because there is evidence that they do not actually exist. It was assumed that bismuth has five conduction electrons per atom.

Clearly the Fermi momenta derived in this way do not fit the free electron model. A glance at the intensity points in Fig. .14 reveals why. The parabolic distributinns are sitting on top of broad backgrounds. These backgrounds are presumably due to nositirnns annihilating with core electrons. The dashed curves in Fig. 14 are Gaussian curves which give a good fit to the far tail points of the intensity distributions. A single Gaussian curve was used for all the pressures. The height of the intensity curves were adjusted so that this Gaussian 
curve represented the same percentage area for all the pressures, about $28 \%$. If it is assumed that this Gaussian curve is a reasonable approximation to the core annihilations, and if this contribution is subtracted from the measured intensity curves and the remainders fit with parabolas, the Fermi momenta which are obtained are those shown in Fig. 19: As can be seen, the agreement is improved considerably. The remaining lack of agreement will be discussed later.

The contribution of the background to the slope, density of states, and number of states are shown as the solid lines in Figs. 15-17. A collection of the densities of states at large angles for all pressures are shown in Fig. 20. The curved line is the same Gaussian as in Fig. 16. If the straight dashed line in Fig. 20 is assumed to be the contribution due to core electrons, the Fermi momenta which are obtained are about $2 \%$ higher than if the Gaussian is used.

The number of electrons annihilating per atom are shown in Fig. 21. Since there are known to be five conduction electrons annihilating per atom must increase from about 1.5 at 0 kbars to about 3 at 95 kbars. All these electrons must be 5 d electrons. The other core electrons are much too strongly localized to annihilate fith the positror. The dashed line in Fig. 21 gives the number of electrons that is implicitly assumed by the Gaussian core correction. It should be remarked that the points. should only be considered as lower limits to the number of electrons because of the approximations that have been made. An examination of the figure reveals that the contribution of the core probably has not been taken completely into account for the three highest pressures. I'is is not unexpected. the average interatomic distance 


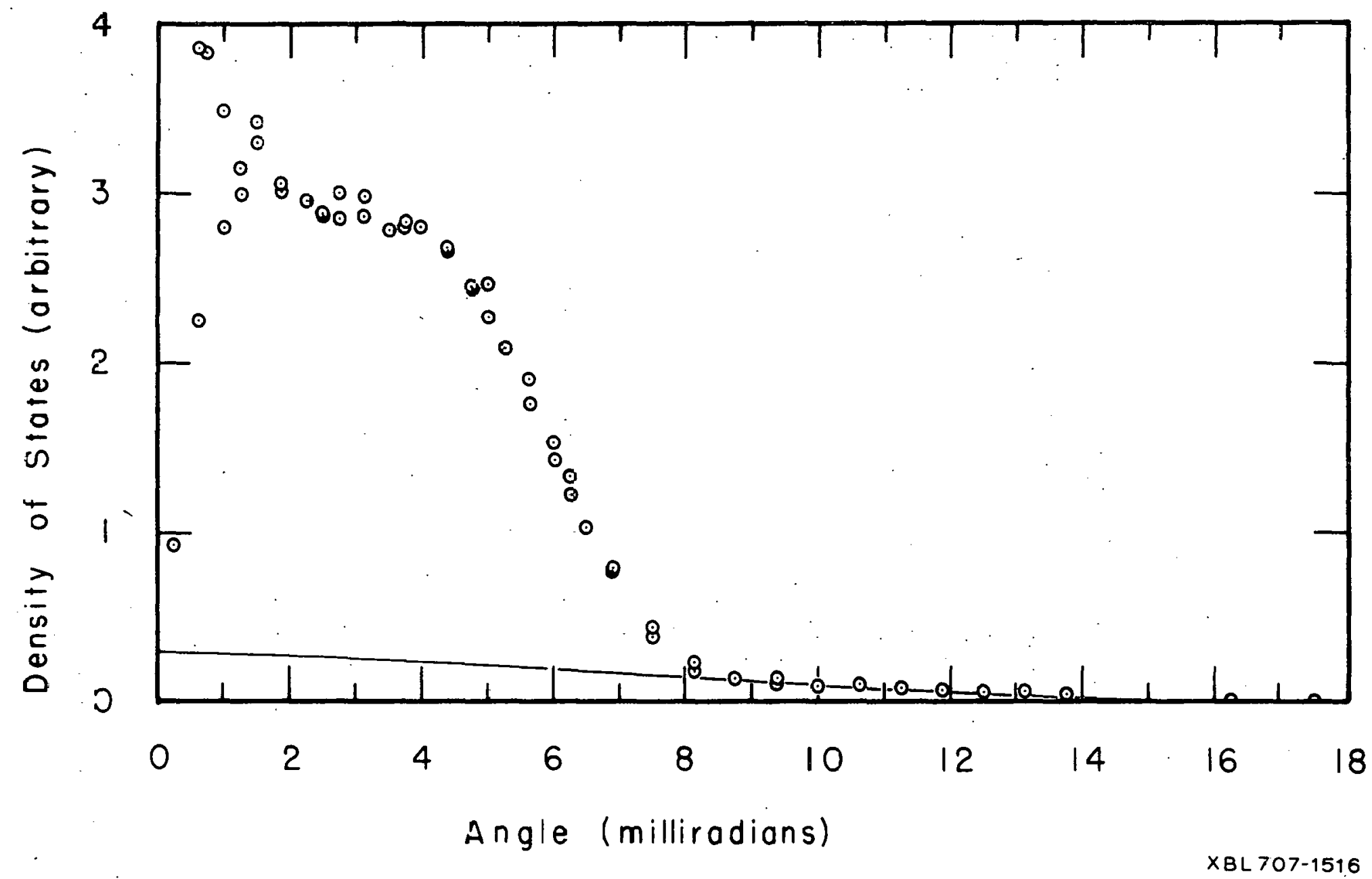

Fig. 16. Density of 0zcupied States in Bismuth at 0 kilobars. 


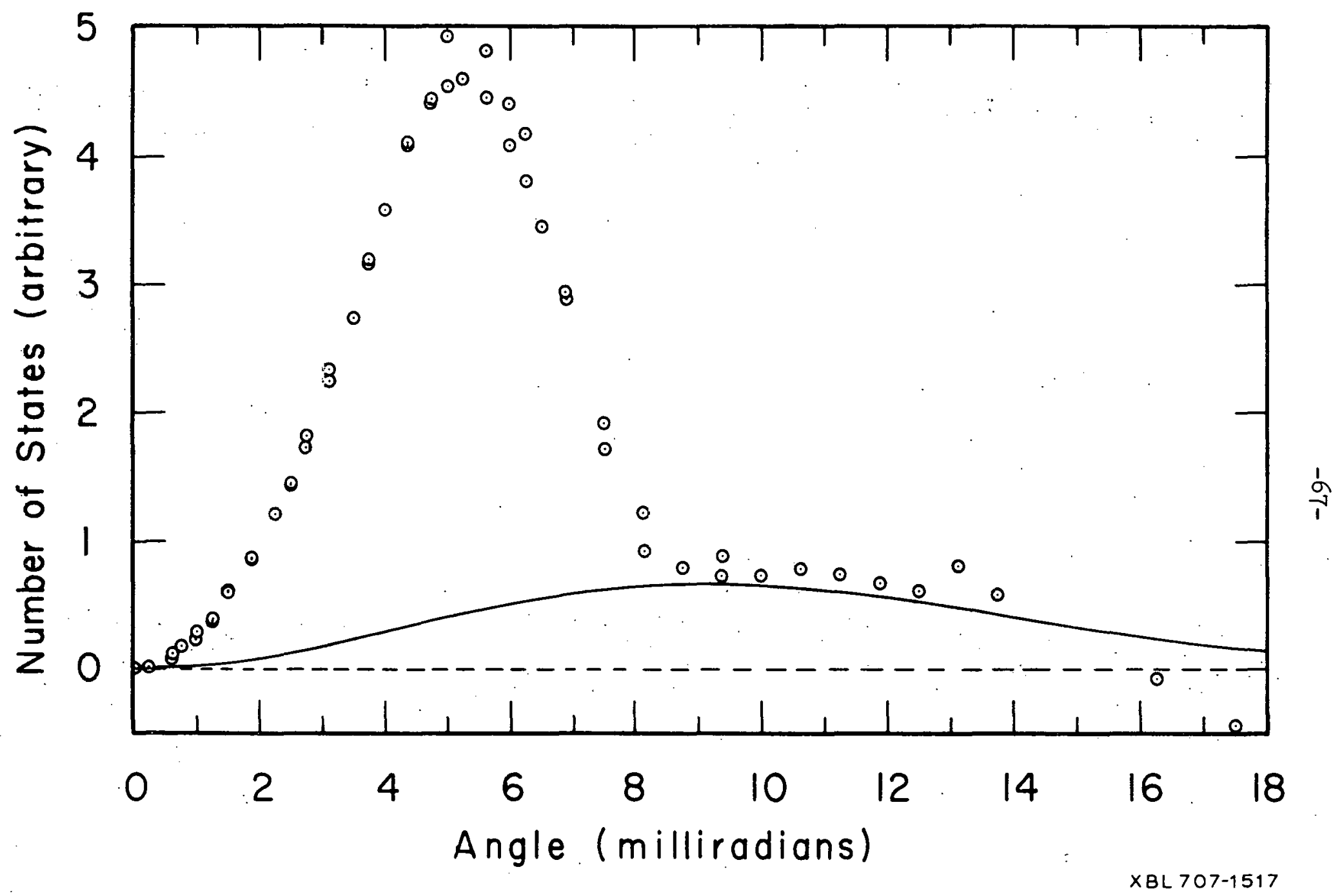

Fig. 17. Number of Occupied States in Bismuth at 0 kilobars. 


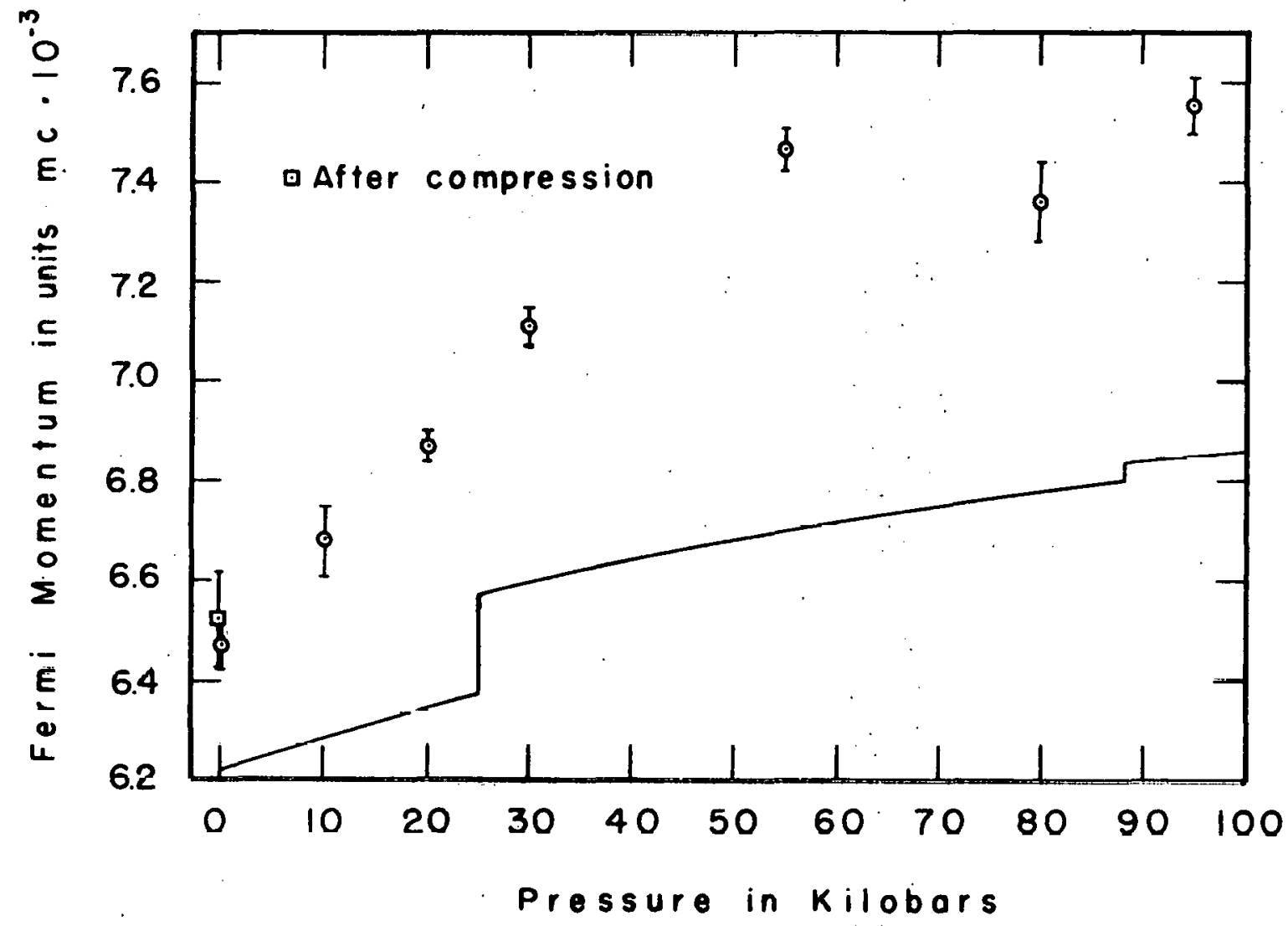

$X B L 707-1496$

Fig. 18. Uncorrected Fermi Momenta of Bismuth. 


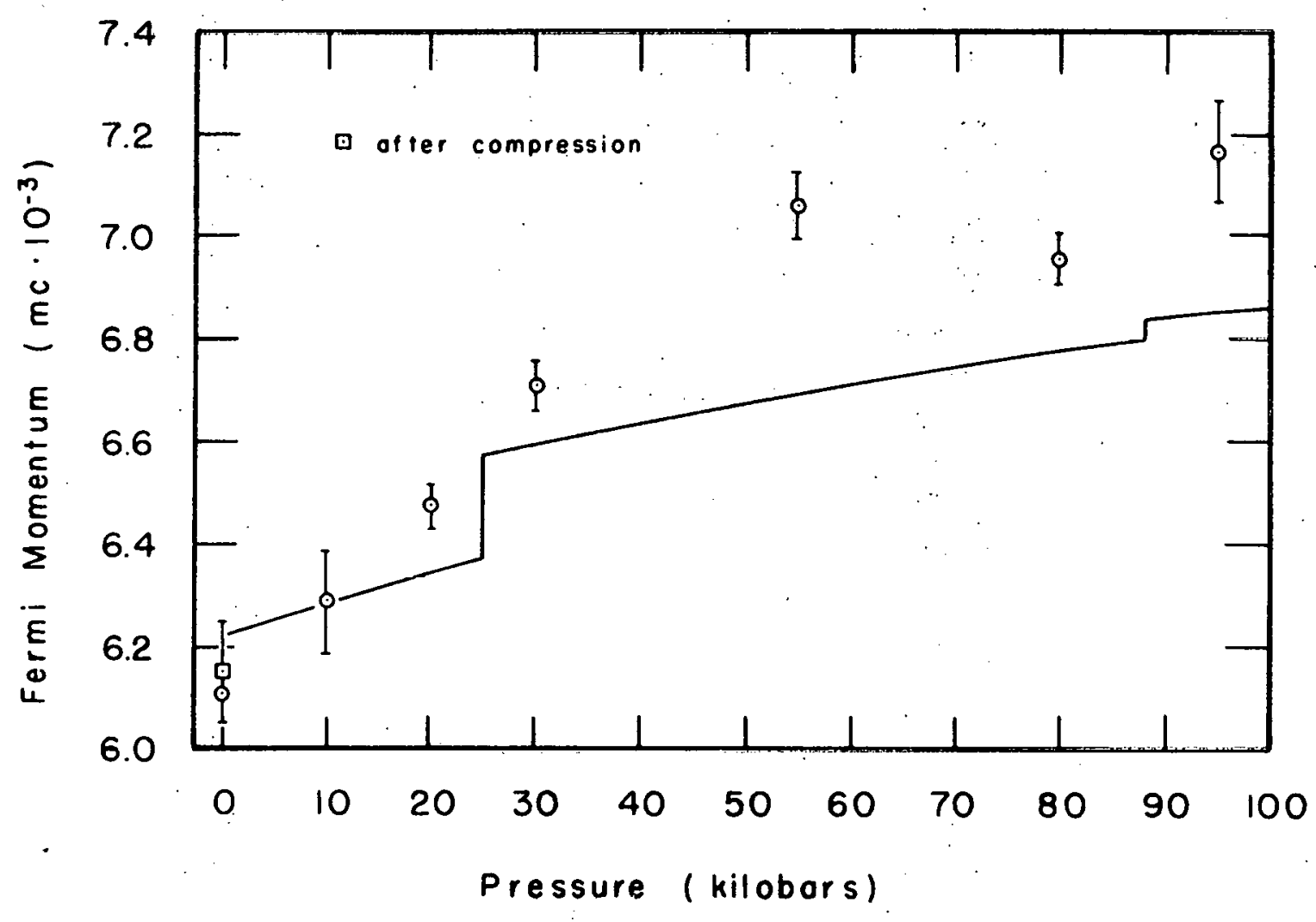

XBL 707-1495

Fig. 19. Corrected Fermi Momenta of Bismuth. 


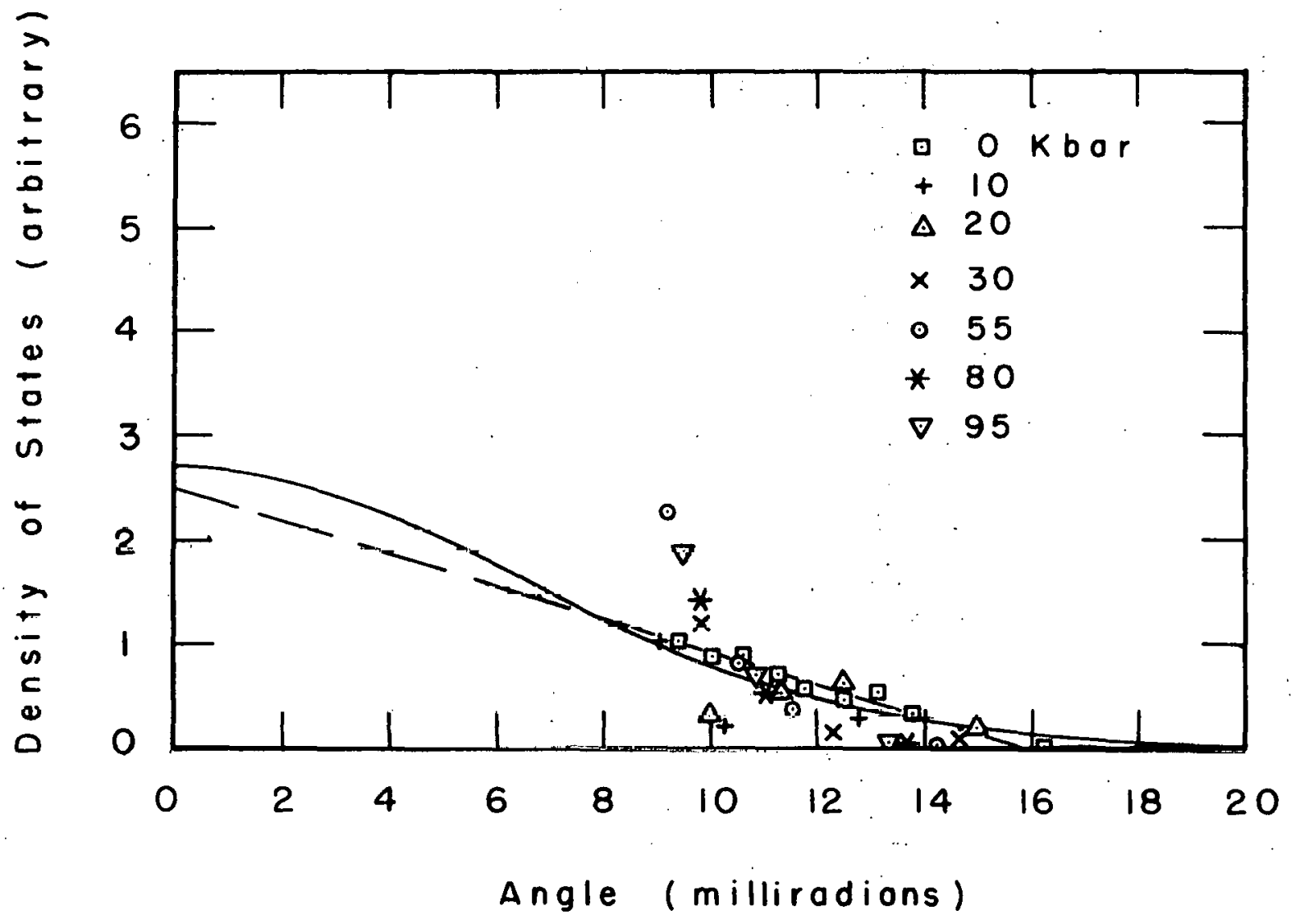

XBL $707-1494$

Fig. 20. Density of States at High Momenta in Bismuth. 


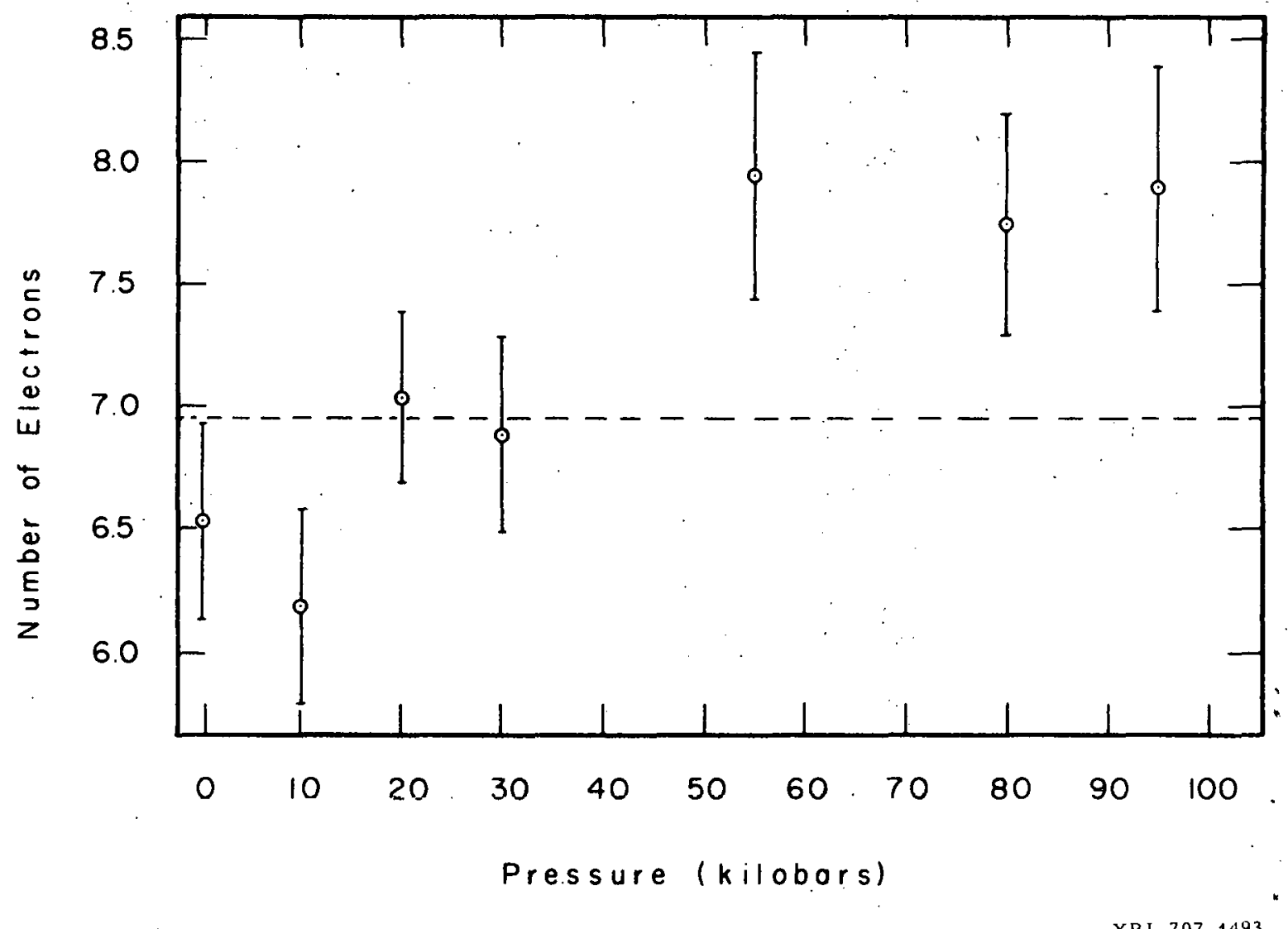

$X B L 707-1493$

Fig. 2l. Number of Annihilating Electrons per atom in Bismuth. 
changes by about $10 \%$ on compression from 0 to 95 kbar. The concentration of $5 \mathrm{~d}$ electrons at interatomic distances must, therefore, change considerably. This effect alone is enough to account for the difference between the theoretical and the experimental curves. The $55 \mathrm{kbar}$ point will still be somewhat high, but this must almost certainly just be due to random experimental scatter. There is no indication that there is anything unusual about the 55 kbar intensity curve.

In summary, the bismuth data is consistent with the free electron theury of metals. The differences between them can be explained by the participation of the 5 d core electrons in the annihilation process. Nothing unusual was noticed in going from one phase of bismuth to another.

\section{Ytterbium}

The positron source for the ytterbium experiment consisted of about I mCi of "carrier free" $\mathrm{Na}^{22} \mathrm{Cl}$ from New England Nuclear Corp. which was evaporated onto a $1 . / 4 \mathrm{mil}\left(0.005^{\circ} \mathrm{mm}\right)$ thick disc of Mylar* about: $1 \% / 4$ in. $(0.645 \mathrm{~cm}$ ) in diameter. This was placed between two discs of ytterbium metal $7 \mathrm{mil}(0.178 \mathrm{~mm})$ thirk and just under 5/16 in. $(0.794 \mathrm{~cm})$ diameter. The metal discs were epoxied together to form a sealed source. The ytterbium which was used was nominally $99.9 \%$ pure. It was purchased from liesearch Chemicals Co. as $10 \mathrm{mil}(0.254 \mathrm{~mm})$ thick foil and was then rolled to $7 \mathrm{mil}(0.178 \mathrm{~mm})$ thickness. The discs were then punched from this sheet.

All data points were corrected for background, angle attenuation and half life. The ytterbium intensity curves could not be reasonably fit by a polynomial in the square of the angle. Even a nineteenth 
degree polynomial in the square of the angle failed to give a fit in which the data points did not systematically deviate from the fitted curve. A slightly different approach was used, therefore. The points were fit with a polynomial in the absolute value of the angle. A seventh degree polynomial was found to give an adequate fit. In spite of the fact that the first method gave significantly poorer fits to the data, the position of the center of the curve which it yielded differed by an average of only about $0.01 \mathrm{mr}$ from that given by the second method. The second method does have one drawback; it does not have zero slope at zero angle. This severely limits the usefulness of the derivatives of the fitted curve in the data analysis. The intensity curves taken at 0 and 85 kbars are shown in Fig. 22. These curves have been normalized to equal areas.

The derivatives of the intensity curves were obtained by the previously explained interpolation technique. The derivatives as a function of angle for two pressures are shown in Figs. 23a and b. It is observed that the initial portion of each of these curves is linear with angle, ao prcdicted by the fret eleclrun theory. This implies that the measured curve is parabolic in this region. Various numbers of points centered about zero angle were fit with parabolas. These fittings revealed that the measured points deviated randomly from the fitted parabola provided that no points were used beyond an angle which.. corresponded to the maximum in the slope. Figure 24 shows the Fermi momenta which were obtained from the widths of the fitted parabolas. The errors were estimated from the scatter of this width as different numbers of points were included in the fit. The two theoretical curves 


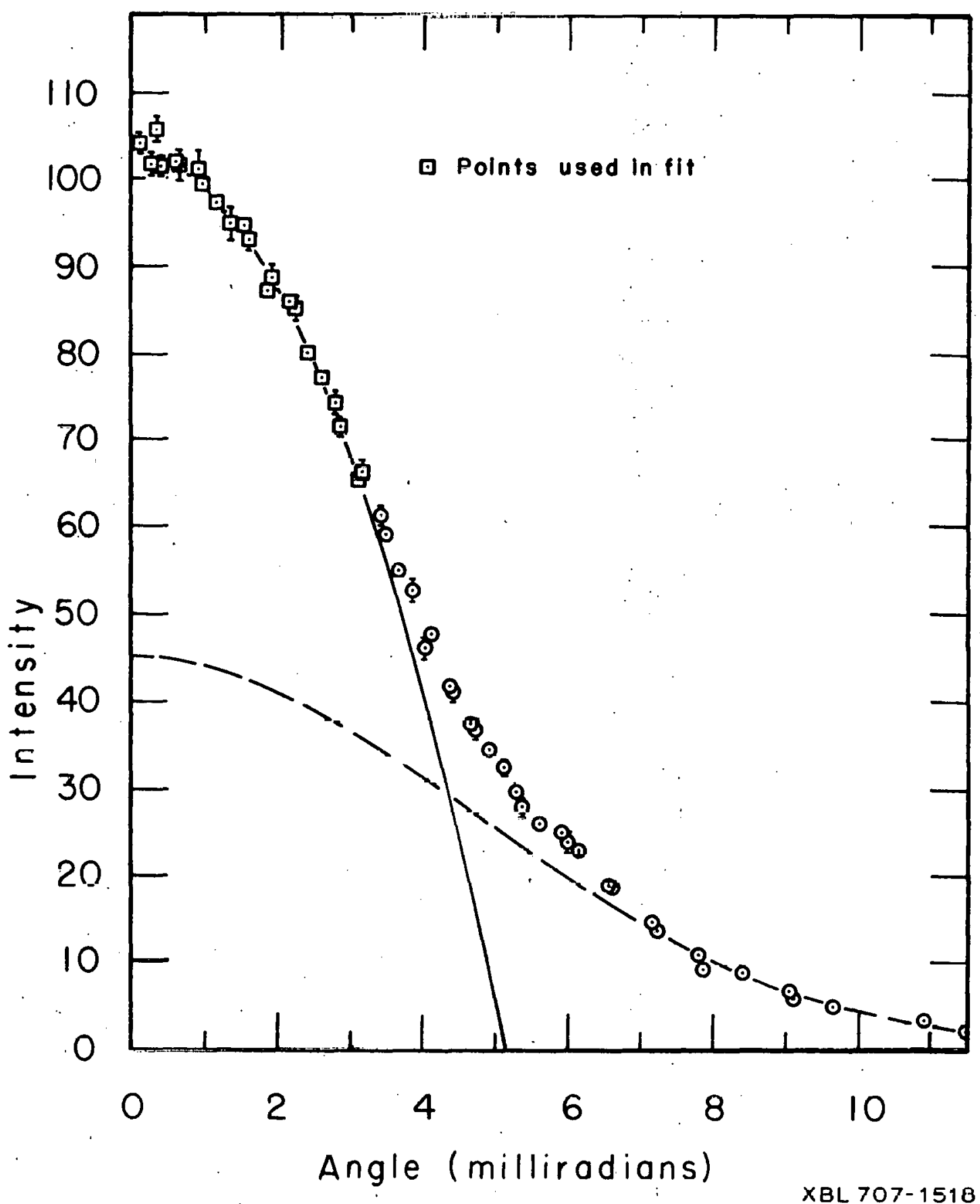

Fig. 22a. Angular Correlation Curve from Ytterhium at n kilobars. 
$-75-$

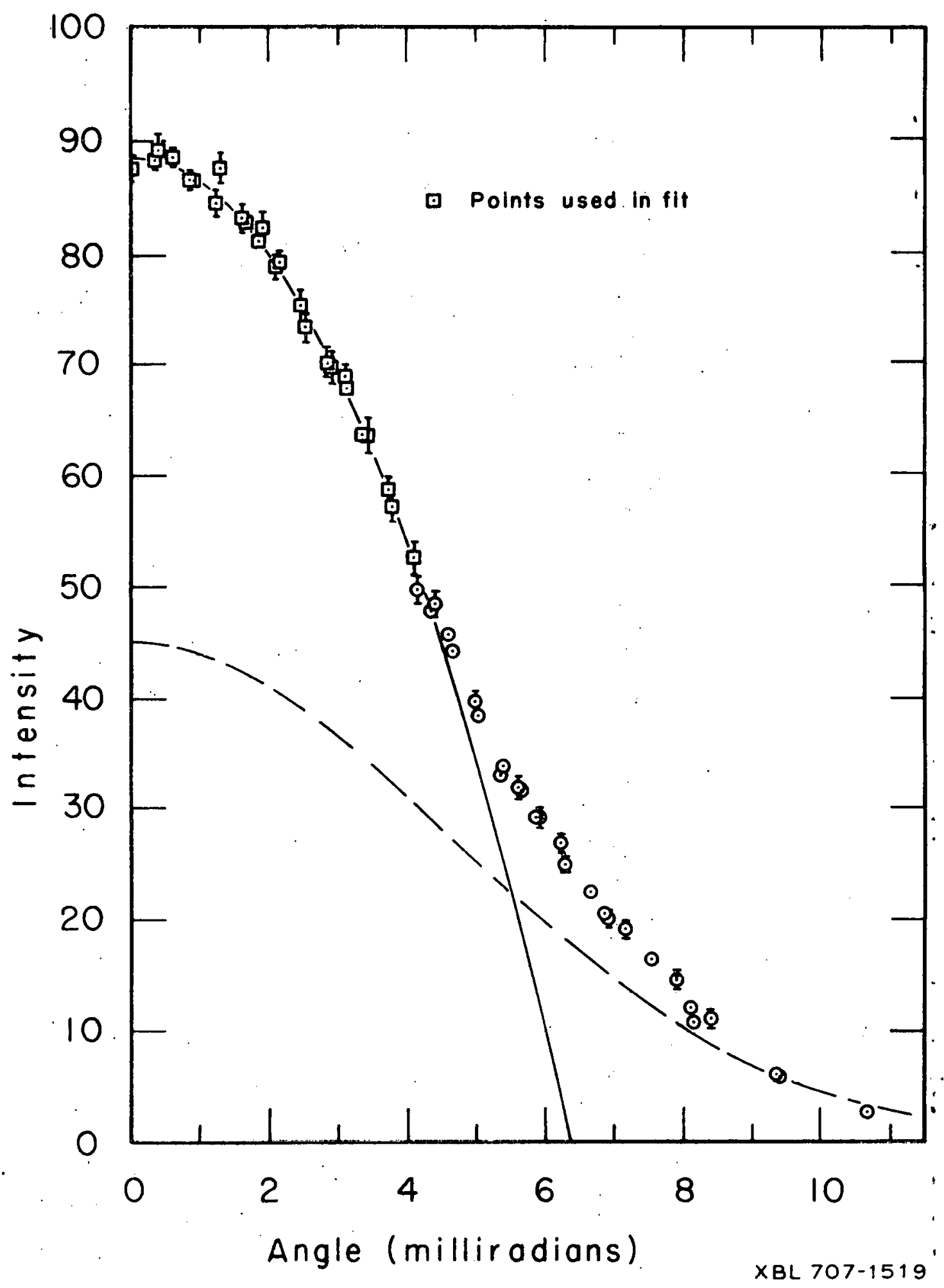

Fig. 22b. Angular Correlation Curve from Ytterbium at 85 kilobars. 


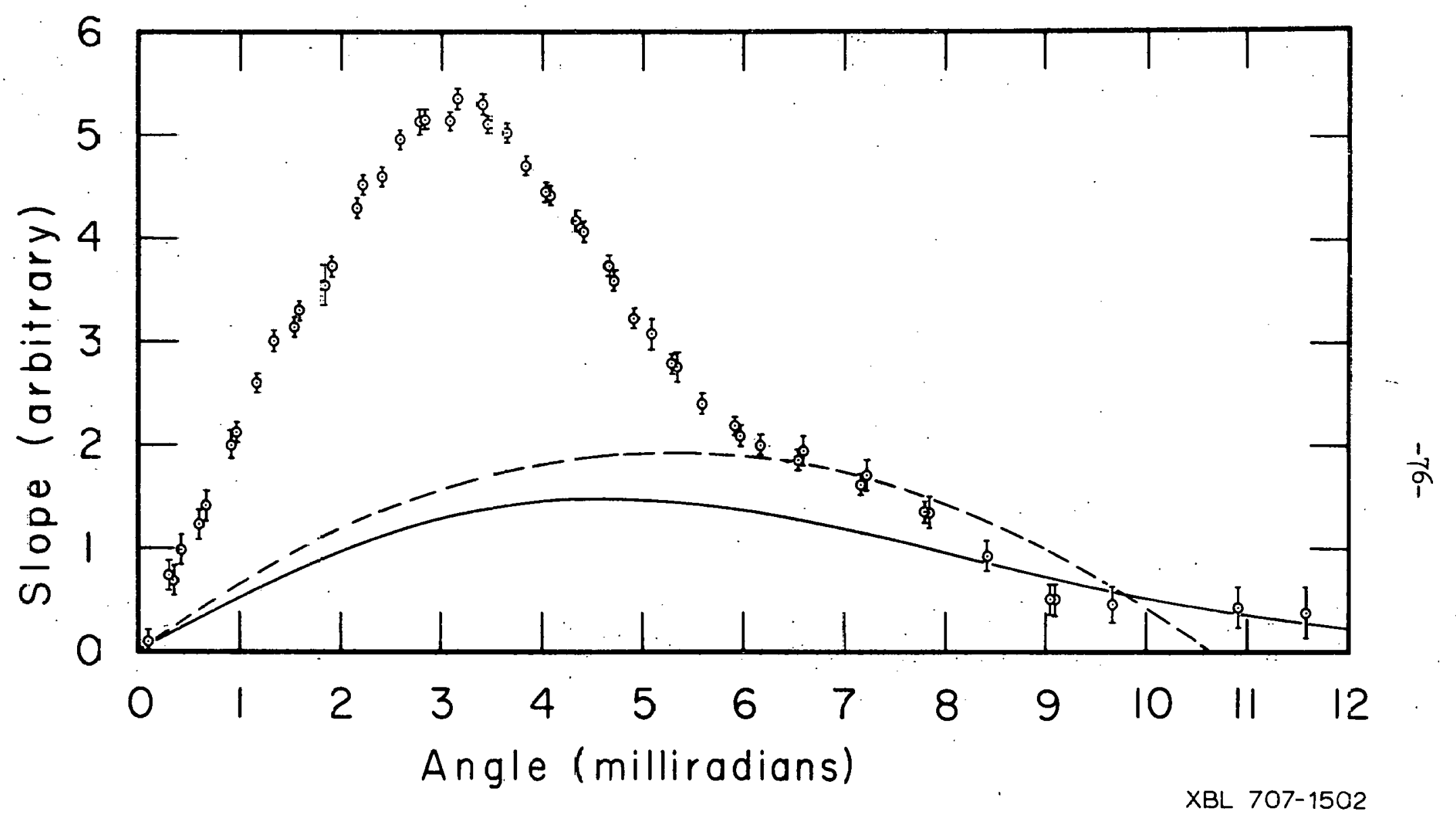

Fig. 23z. Slopes of the 0 kilobar Ytterbium Curve. 


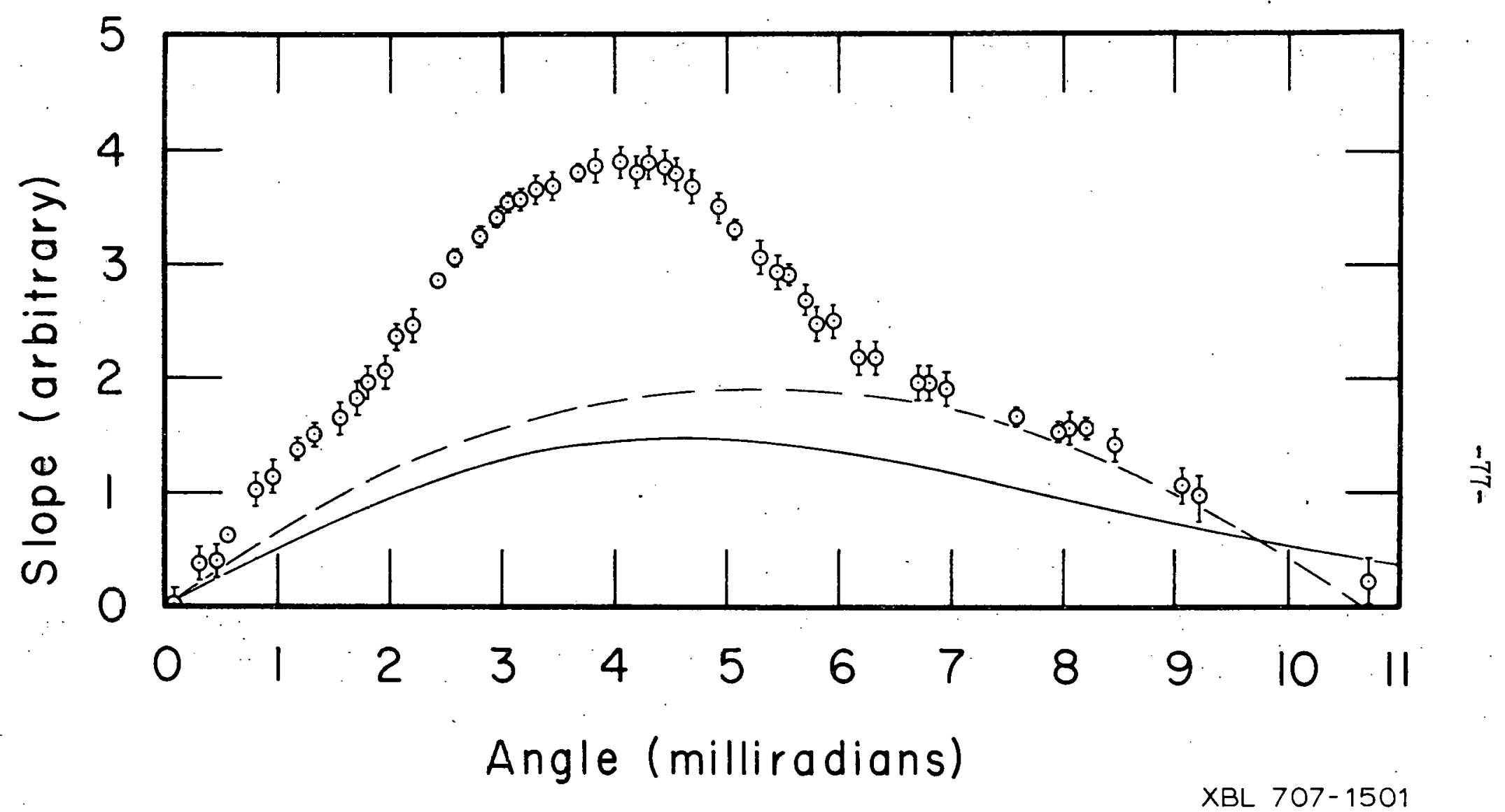

Fig. 23b. Slopes of the 85 kilobar Ytterbium Curve. 


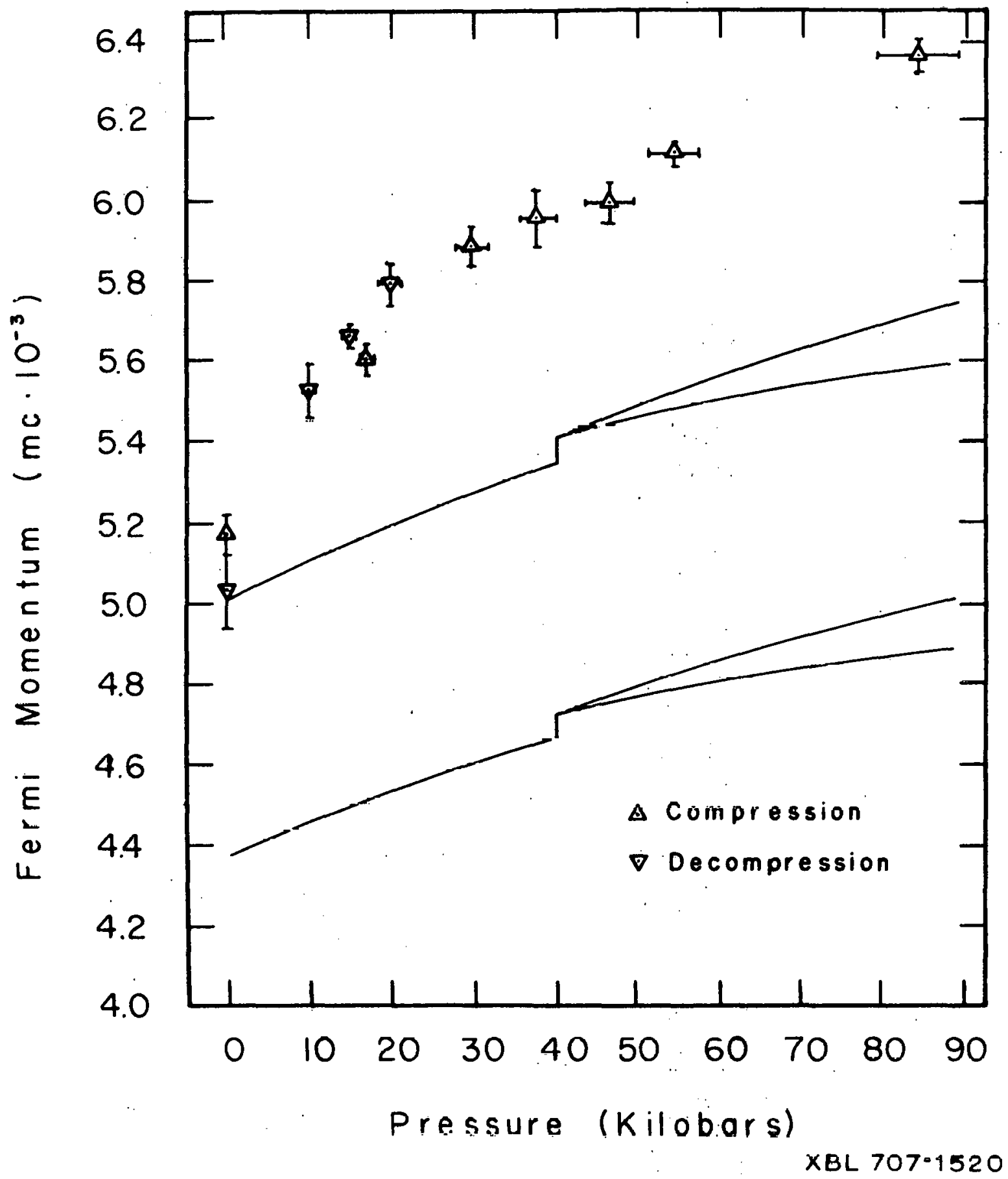

Fig. 24. Uncorrected Termi Momenta of Ytterbium. 
which are shown are from the free electron theory assuming two and three electrons per atom. An initial density of $6.98 \mathrm{~g} / \mathrm{cm}^{3}$ was assumed for $\mathrm{Yb}$ metal for the calculation of the theoretical curve. The volumes which were used in the calculation were those of Stevens. ${ }^{41}$ This data only extends to about $40 \mathrm{kbars}$. Two different approximations were made for the compression of Yo from 40 to 85 kbars. For the first approximation the compressibility of $\mathrm{Yb}$ from 40 to 85 kbars was assumed to be similar to that of nearby "normal" rare earths from 0 to $45 \mathrm{kbars}$. This is not unreasonable since $\mathrm{pb}$ is thought to become a normal rare earth at the $40 \mathrm{kbar}$ phase transition. This approximation almost certainly does not overestimate the compressibility and may underestimate it. For the second approxination it was assumed that $\mathrm{xb}$ has the same density at $85 \mathrm{kbar}$ as it would if it were a normal rare earth compressed to $45 \mathrm{kbar}$. This gives a smaller volume and, therefore, a higher Fermi momentum. This approximation probably overestimates the compressibility。 Fortunately these theoretical curves are not strongly dependent on the volume.

Clearly the Fermi momenta points do not fit either the two or three electron curves. The fact that the experimental values are too large is not unusual. This is due to the fact that core annihilations have not yet been taken into account. At very high momenta presumably the only contribution to the measured curve is that from annihilation of the positrons with electrons in core states. This contribution should be relatively independent of pressure. Figure 25 shows the density of state data for all pressures at momenta greater than about $7.2 \times 10^{-3} \mathrm{mc}$. All data have been normalized to each other by setting the areas of the 


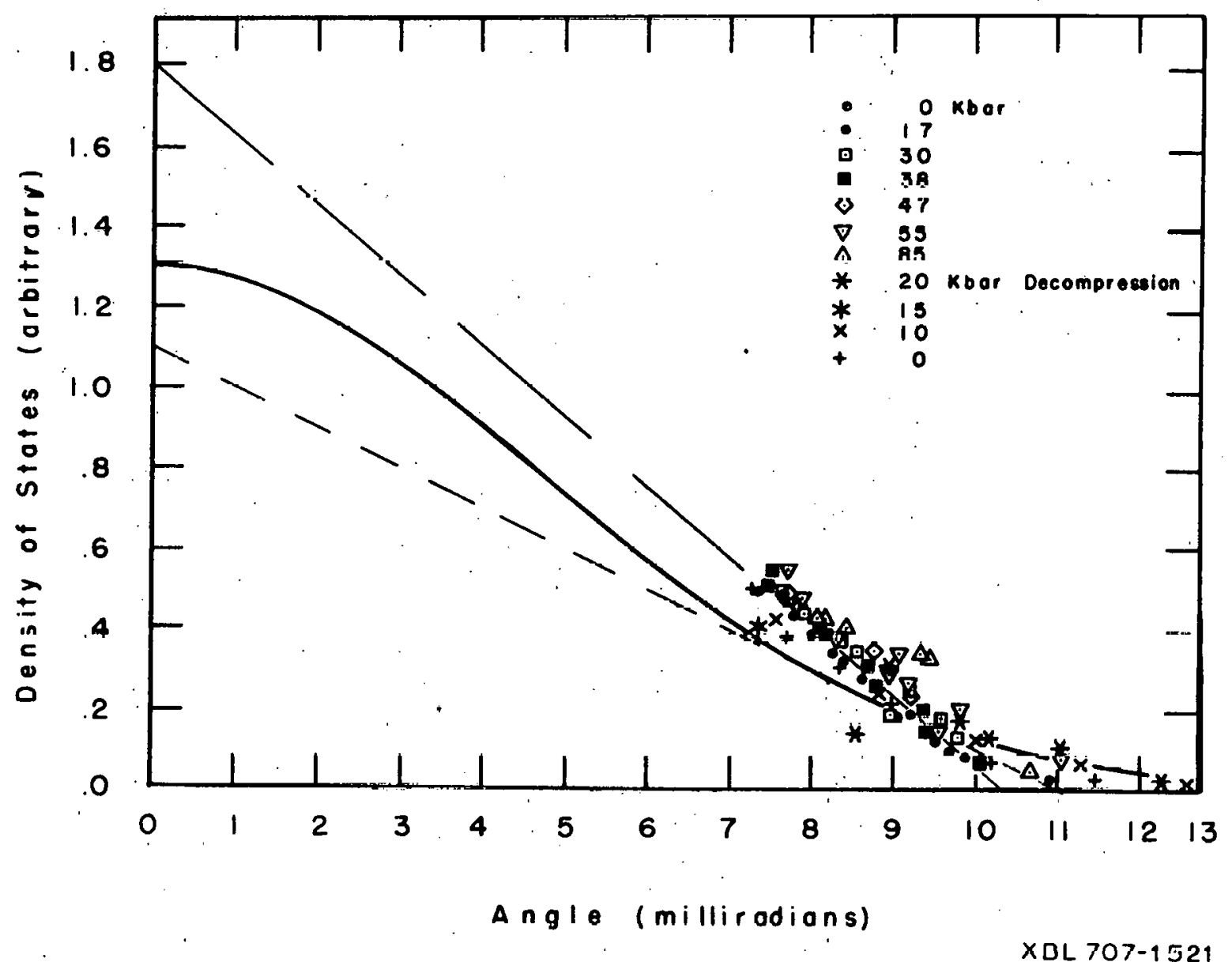

Fig. 25. Density of Ștates at High Momenta in Ytterbium. 
measured curves equal to each other. As was discussed earlier, this normalization is equivalent to normalizing to the same number of electrons annihilating. These points suggest a straight line. Three different empirical contributions of core annihilations to the measured spectrum have been calculated. These are represented by the two dashed straight lines and the curved line in Fig. 25. The curved line is obtained from a Gaussian curve which gives a good fit to the far tail points of the intensity curves. The dashed and solid line in Fig. 23 represent the contributions to the slope corresponding to the upper dashed line and the Gaussian curve in Fig. 25.

The measured intensity points were corrected for these contríbutions and the points fit with parabolas. The Gaussian correction to the intensity is shown as the dashed line in Figs. $22 \mathrm{a}$ and $\mathrm{b}$. The intensity curves after this correction are shown in Figs. 26a and b. A fitted parabola is also shown in these figures. The Gaussian correction is about $44 \%$ of the total area of the intensity curves. All three corrections yielded similar corrected Fermi momenta. The greatest differences between the corrected Fermi momenta were for the points at zero and 85 kbars. The Fermi momenta which were obtained using the Gaussian core correction are those shown in Fig. 27. If the density of states is that shown in Fig. 25 by the upper dashed line, the 0 kbar points are about the same as those shown, and the $85 \mathrm{kbar}$ point is about 0.1 unit greater. If the density of states used for the correction is that shown by the lower dashed line, the 0 kbar points are about 0.2 units greater, and the $85 \mathrm{kbar}$ point about the same as that shown in Fig. 27. All other points are about the same with each of the three corrections. 


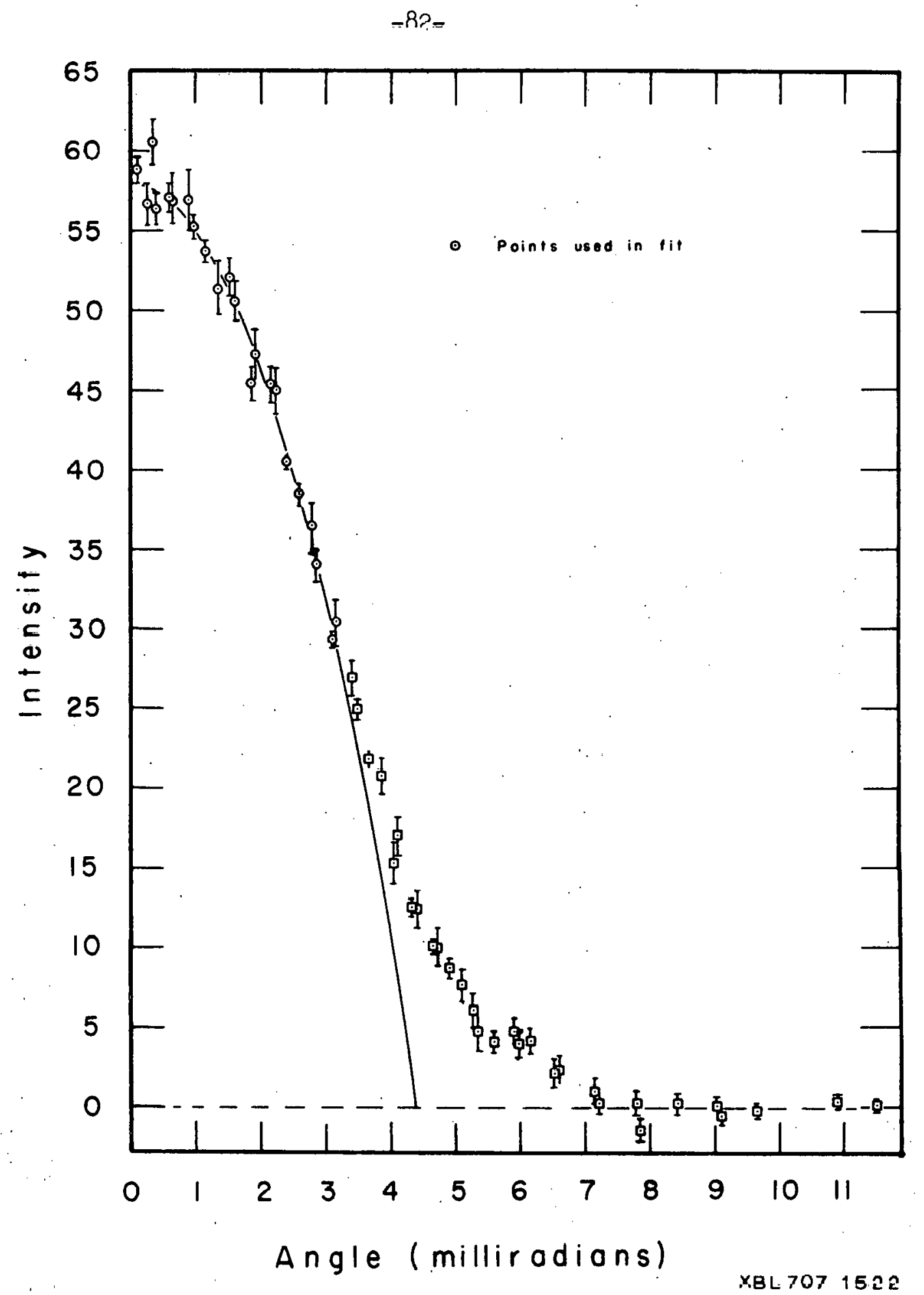

Fig. 26a. Corrected Angular Correlation Curve from Ytterbium at 0 kilobar. 


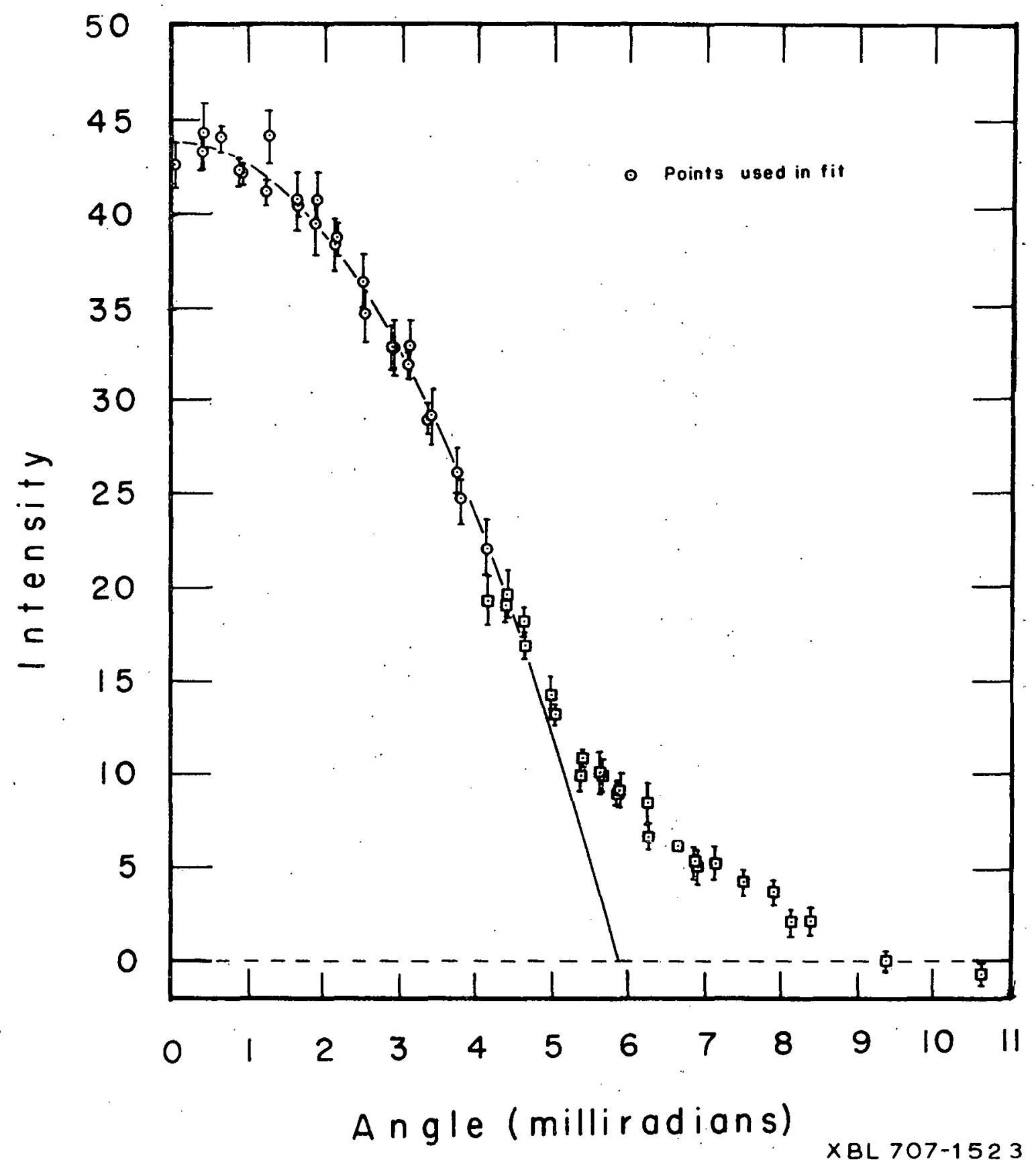

Fig. 26b. Corrected Angular Correlation Curve from Ytterbium at 85 kilobars. 


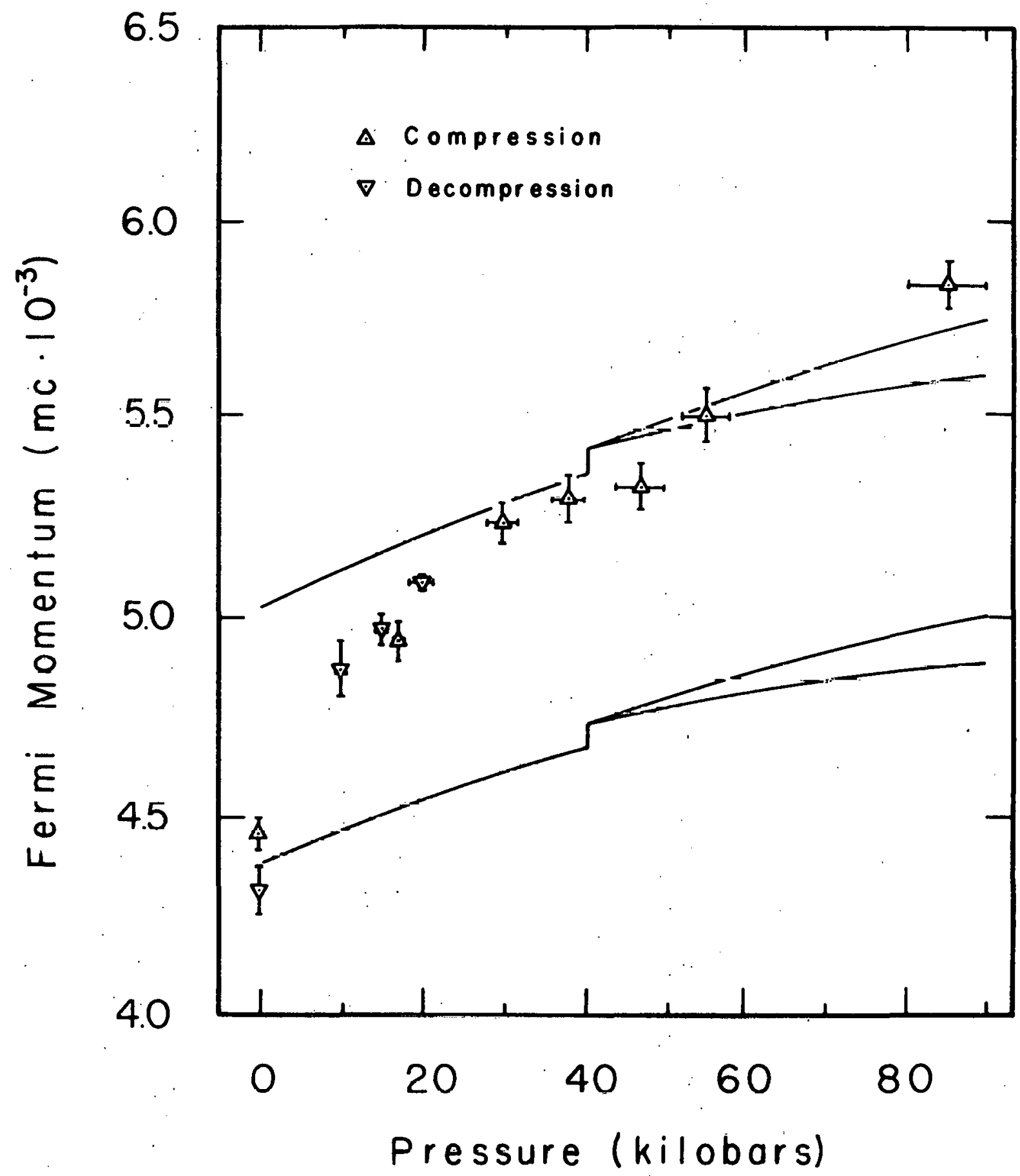

XBL TO7-1524

Fig. 27. Corrected Fermi Momenta of Ytterbium. 
The shift in the Fermi momentum that is caused by any one of these corrections for the core states is clearly in the right direction, and is relatively insensitive to the exact correction used.

All of the slope versus angle plots seemed to be linear for the first $2 \mathrm{mr}$. The slope at $2 \mathrm{mr}$ was, therefore, calculated from a straight line which was fit through the origin to those points between 0 and $2 \mathrm{mr}$. Using the assumption that this slope is characteristic of the conduction electrons, the number of electrons annihilating at each pressure were calculated. The results are shown in Fig. 28. The error bars reflect the error in measuring the slope of the curve. The error in measuring the area of the curve is only a few tenths of a percent, which is negliglble compared to the error in deriving the slope.

The first thing that becomes apparent upon examination of Fig. 28 is that the number of electrons is larger than might be expected. At o kilobars it is well established that ytterbium has only two conduction elecurons. Hall effect measurements ${ }^{42}$ and positron lifetime measurements are consistent with a picture of two conduction-electrons in yllerbium. Magnetic susceptibility measurements 43 show that there are virtually no unpaired electrons. The conclusion must be reached, then, that positrons in $\mathrm{Yb}$ at atmospheric pressure anihilate, on the average, with about 1.6 core electrons for every 2.0 conduction electrons. This is not unreasonable. It is known that the lifetime of positrons in $\mathrm{Yb}$ is about $30 \%$ longer than in its normal, three conduction electron, rare earth neighbors. 49 It is known from theory that the positron lifetime should vary faster than the density of electrons in real space for this range of densities. 44 Theory also yields a lifetime which is an order 


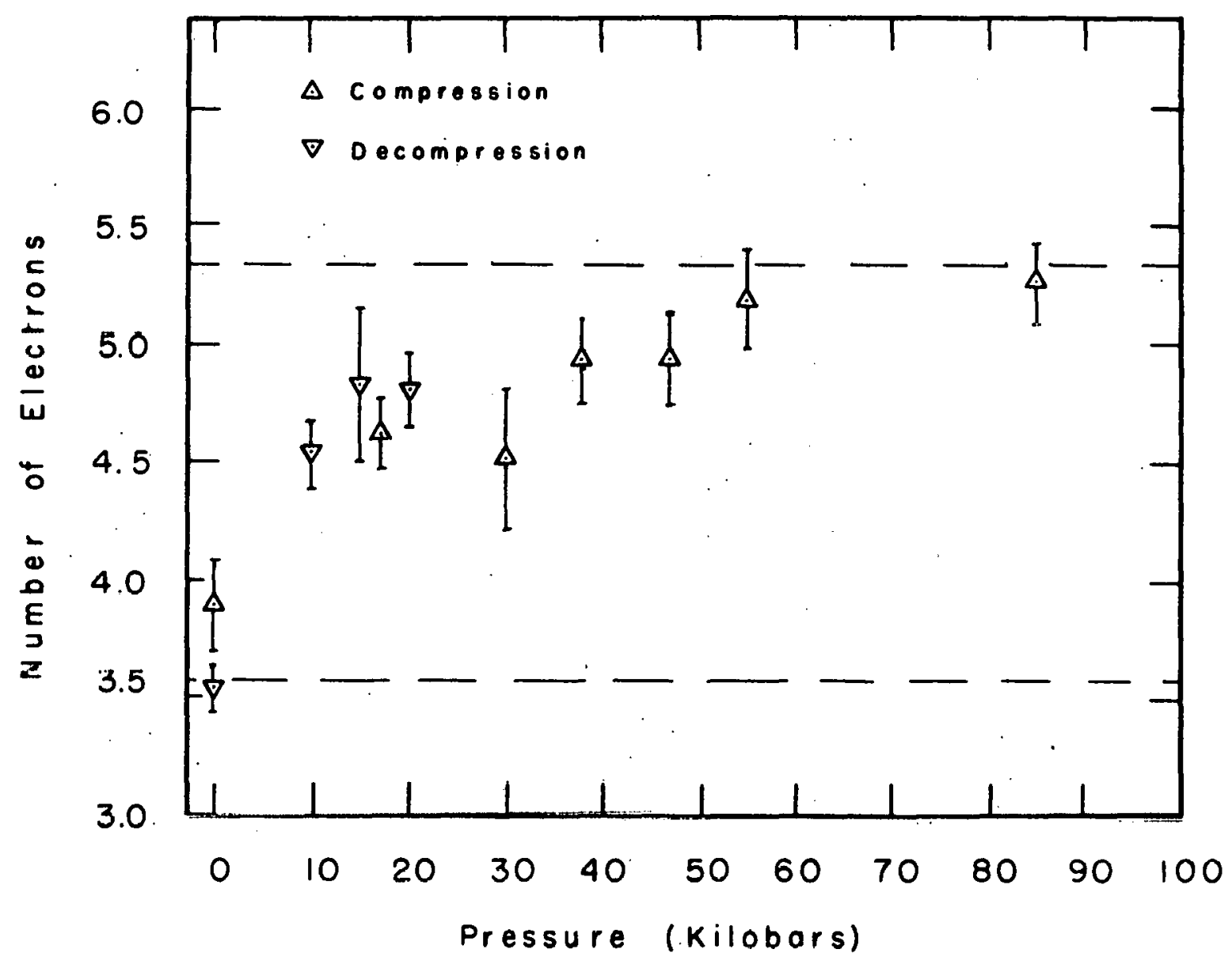

Fis. 28. Number of Annihilating Elestions yer Alum in Ytterbium. 
of twice as long as the measured lifetime. It becomes apparent from these facts that the number of core electrons which annihilate with the positrons must be the same order of magnitude as the number of conduction electrons. The total number of electrons which is implicitly assumed by the Gaussian correction to the intensity curve is shown by the horizontal dashed lines in Fig. 28. The upper line assumes three conduction electrons and the lower line, two. These lines appear to be consistent with the points shown. The estimates made here of the number of electrons per atom annihilating, however, should only be taken as lower limits because of the assumptions involved.

The next question that occurs is that of which core electrons annihilate with the positrons. The positron, being a positively charged species, tends to stay as far away as possible from the positively charged nuclei. At these large distances it will be well shielded from the nucleus by the core electrons. Only electrons which have a good probability of being at large distances from the nuclei will have a good chance of annihilating with the positron. Of the core electrons in $Y b$, those electrons in the $5 p$ and $5 s$ states will have the greatest probability of being at the distances which are involved, about four atomic units. 45 The 5 p have somewhat higher probability than the $5 \mathrm{~s}$ electrons. The probability of a $4 f$ electron being at these distances is an order of magnitude liess. Taking into account the number of electrons occupying each kind of state and their relative probabilities of being at large distances from the nuclei, it can safely be said that most of the core electrons which annihilate with the positrons are electrons in $5 p$ states. A few $5 s$ and $4 f$ electrons also annihilate. 
It is to be expected that the number of core electrons annihilating will change only slowly with pressure.

Figure 28 shows that the total number of electrons annihilating with the positrons is much greater under the application of even as little as $10 \mathrm{kbar}$ than it is at $0 \mathrm{kbar}$. If this result is compared with Fig. 27, the explanation which suggests itself is that under pressure an electron, or an appreciahle fraction of an electron per a,tom, io bcing promoted lu a ja conduction bund. 'lhe f'urther slight increase in the number upon the application of more than 40 kbars of pressure is just die to greater annihilation with the core electrons. Only the $4 \mathrm{f}$ electrons are close enough energetically to the $5 \mathrm{~d}$ states to be promoted to a $5 d$ state.

In order to explajn this data in a manner that is consistent with previous measurements of electrical resistivity, Hall effect, and magnetic susceptibility, the band structure of f'i.g. 29 is proposed. The probable widths and positions of the hands as a funotion of intcrabomic distance are shown. $r_{p}$ is the interatomic distance at some pressure, $p$. The solid lines show the proposed energies for an $\mathrm{Yb}^{2+}$ core. The dashed lines show the energies for an $\mathrm{Yb}^{3+}$ core. If an electron is transferred from a $4 f$ state to a $5 d$ state, the levels of all the other states shift. Thin io because the outer states now see a core of $3+$ charge instead of 2+ charge. Tt is effects of this type which make diagrams of the type shown here of limited usefulness. With this in mind the following explanation is offered for the positive pressure coefficient of resistivity of $\mathrm{Yb}$ up to the fcc-bcc phase transition at $40 \mathrm{kbar}$. 


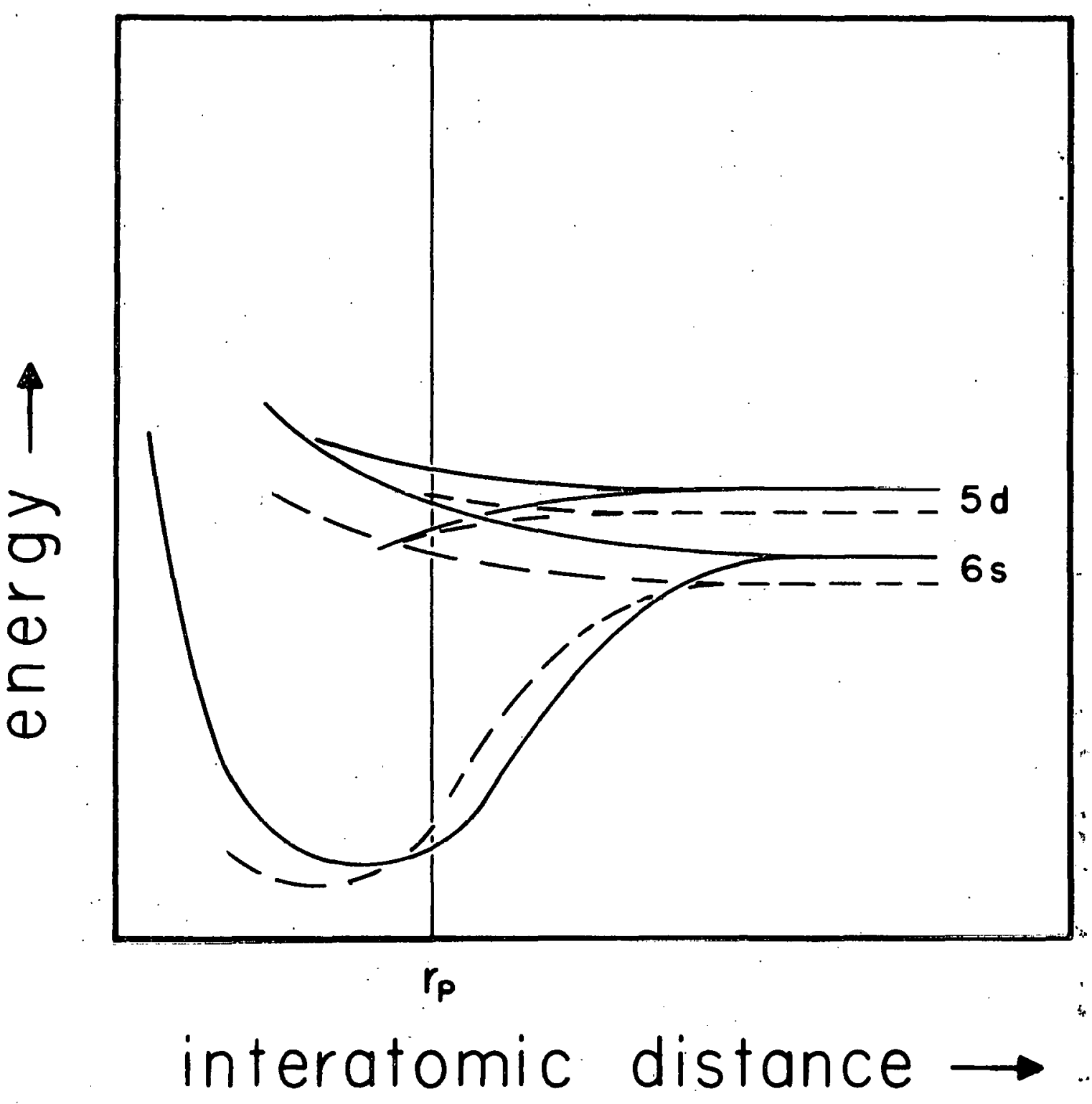

XBL $707-149 i$

Fig. 29. Proposed Band Structure of Ytterbium. 
Magnetic susceptibility measurements by Lock 43 show that about one $\mathrm{Yb}$ atom in 260 has an unpaired electron in $\mathrm{Yb}$ metal at atmospheric pressure. Lock's measurement leaves little doubt that at atmospheric pressure there are about 2.004 conduction electrons per $\mathrm{Yb}$ atom. It is known, however, from Hall effect measurements that the main carriers in $\mathrm{Yb}$ at atmospheric pressure are holes. ${ }^{42}$ The number of holes appear from the Hall effect measurements to be almost independent of temperature. These facts indicate that the $5 \mathrm{~d}$ band almost certainly overins with the $6 \mathrm{~s}$ band and that the holes have very much lower effective masses than the electrons. For holes near the top of a broad band such as the $6 \mathrm{~s}$ band in $\mathrm{Yb}$, effective masses of 0.01 or less are not uncommon. On the other hand, electrons in a narrow band, such as the 5d band, may have effective masses of several hundred. If the relaxation times are similar for the electrons and holes, the holes can have an effect on the conductance which is. four orders of magnitude greater than that of the slectrons.

The conductivity of fec $\mathrm{Yb}$, then is determined mainly by the very light holes at the top of the $6 \mathrm{~s}$ band. The electrons in the narrow $5 \mathrm{~d}$ band have high effective mass and contribute very little to the conductivity. It appears that under the application of pressure it becomes energetically favorable for $4 f$ electrons from at least some of the atoms to transfer to 5d states. This causes the top of the $6 \mathrm{~s}$ hand tin fa.t below the Fermi level. This causes the destrüction of holes as electrons fall into the $6 \mathrm{~s}$ band. As pressure is further increased, it becomes favorable for a larger percentage of $4 \mathrm{f}$ electrons to transfer to $5 \mathrm{~d}$ states. This continues until all the atoms have only thirteen 
$4 f$ electrons. It is not energtically favorable for a second $4 f$ electron to be transferred to a $5 \mathrm{~d}$ state, creating a $4+\mathrm{Yb}$ core. This decrease in the number of holes with pressure causes the observed increase in resistivity. The negative temperature coefficient of $\mathrm{Yb}$ from 20 to 40 kbar may be interpreted in the following manner. As the temperature is increased, increased thermal excitation of electrons from the top of the $6 \mathrm{~s}$ band to above the Fermi energy allow the holes which are created in the $6 \mathrm{~s}$ band to conduct electricity. The band gap measurement of Souers ${ }^{6}$ may then be interpreted as measuring the average energy difference between the top of the $6 \mathrm{~s}$ band and the Fermi surface, not an energy gap in the normal sense.

This explanation is also compatible with the resistivity-pressuretemperature measurements on $\mathrm{Yb}$ by McWhan et al. ${ }^{75}$ At the lowest temperatures which they obtained $\left(2^{\circ} \mathrm{K}\right)$, the conductivity of fcc $\mathrm{Yb}$ at.. atmospheric. pressure is about four and one half orders of magnitude greater than it is at $45 \mathrm{kbar}$. This is consistent with the estimated effective masses of holes and electrons in the $6 \mathrm{~s}$ and $5 \mathrm{~d}$ bands.

After the fcc-bcc phase transition, the character of the $5 \mathrm{~d}$ band is probably very different. It is likely that the wavefunctions of the $5 \mathrm{~d}$ bands still have a great deal of $5 \mathrm{~d}$ atomic orbital character about them, and are, therefore, quite sensitive to the geometry of the surrounding atoms. From the previous arguments on the effective masses of the electrons in the two bands, it seems most likely that the metallic conductivity of bce $\mathrm{Yb}$ is due to holes, or possibly even electrons, in the $6 \mathrm{~s}$ band. This would require that the lowest $5 \mathrm{~d}$ band be shifted sufficiently. far down in energy relative: to the 
$6 s$ band so that the Fermi energy lies below the top of the $6 \mathrm{~s}$ band. From the data presented here, it can be said with a fair degree of certainty that bcc $\mathrm{Yb}$ does have three electrons per atom in the conduction bands, as had been proposed. The surprising thing is that fcc $\mathrm{Yb}$ at $40 \mathrm{kbar}$ also appears to have three conduction electrons per atom. 


\section{MOSSBAUER EFFECT IN Dy ${ }^{161}$}

A. INTRODUCTION

The Mossbauer effect is a tool which may be used to study the environment of a nucleus. It is known that a large number of rare earth nuclei are excellent candidates for observing the Mössbauer effect. Among these is $\mathrm{Dy}^{161}$, which is the nucleus which was chosen for this experiment.

Early work by Stone ${ }^{52}$ indicated that the Mössbauer effect of Dy ${ }^{161}$ in gadolinium metal at very high pressure is easily measurable at room temperature. His detailed results, however, disagreed with measurements made with the same isotope in other environments by other workers. $53-58$ Since there were several improvements which could easily be made on Stone's apparatus, it appeared profitable to repeat his experiment and, if that were successful, to also extend it to $\mathrm{Dy}^{161}$ in dysprosium metal at high pressures.

\section{B. THEORY}

There are several excellent books and review articles which deal with the Mossbauer effect in detail. 59-61 The account given here only covers the main results of the theory of the Mössbaver effect.

The Mössbauer cffect is a resonant yamna ray scattering phenomenon. The usefulness of the effect follows from the unique set of circumstances which makes the effect possible. Consider a free nucleus initially in an excited nuclear state. Assume that the nucleus spontaneously transforms to the ground state, emitting a gamma ray in the process. Because momentum and energy must both be conserved, the energy of the emitted 
gamma ray, $E$, must be less than the energy of the transition, $E_{0}$, by the recoil energy, $E_{R^{\prime}} \quad E=E_{0}-E_{R} \cdot E_{R}=E^{2} /\left(2 m c^{2}\right)$. $m$ is the mass of the nucleus, $c$ is the speed of light. Conversely, in order to excite a similar nucleus from the ground state to that excited state, a gamma ray of energy $E=E_{O}+E_{R}$ is required. Resonant scattering of these gamma rays cannot take place unless the width of the distribution of the emitted gama rays, $\Gamma$, is larger than the recoil energy. $\Gamma>E_{\Pi^{*}}$ This width is determined by the mean life, $\tau$, of the excited nuclear state through the uncertanty principle, $\Gamma \tau=$. I . It $\tau$ is $10^{-7} \sec \left(\right.$ a typical value), $\Gamma$ is about $10^{-8} \mathrm{eV}$. In comparison, if $E$ is $20 \mathrm{keV}$, and $\mathrm{m}$ is $50 \mathrm{amu}$, then $\mathrm{E}_{\mathrm{R}}$ is about $10^{-3} \mathrm{eV} . \mathrm{E}_{\mathrm{R}} \gg \Gamma$. This recoil energy was thought to make it impossible to perform a normal resonant scattering experiment with gamma rays.

If the nucleus under considesation is nol fret, bul is bunn in a lattice, it cannot gain any arbitrary amount of kinetic energ., $E_{R^{*}}$ The amount of energy the lattice can gain (or lose) is guantized. When a nucleus which is bound in a lattice emits a gamma ray, there is a certain probability that the lattice energy state after the transition will be any one of the states. The salient point is that there is a certain probability that the lattice will not change its energy state. In that case the energy of the emitted gamma ray must be equal to the energy of the nuclear transition. $E=E_{n}$. Under favorable conditions the probability of this happening may be very great. Since $\mathrm{E}$ can equal $\mathrm{E}_{\mathrm{O}}$ for both the emission and absorption process, it is possible to perform the resonant scattering experiment. This was Móssbauer's discovery. 
When this recoilless emission takes place the width of the distribution of the emitted radiation is just that determined by the uncertainty principle, typically $10^{-8} \mathrm{eV}$. This often is less than the splittings in the nuclear energy levels which are caused by magnetic moment-magnetic field, and quadrupole moment-electric field gradient interactions. The energies of the split levels relative to the initial unsplit state due to the quadrupole splitting are

$$
E_{Q}=\frac{e q Q}{4 I(2 I-I)}\left[3 m_{I}^{2}-I(I+1)\right]\left(I+\eta^{2} / 3\right)^{1 / 2}
$$

$m_{I}=I, I-I, \cdots \cdots,-I$

$I=\operatorname{spin}$ of the nuclear state

$Q=$ quadrupole moment

eq $=v_{z z}=\partial^{2} \cdot v^{\prime} / \partial_{z}^{2}, v=$ electric field, $\left|v_{z z}\right|>\left|v_{x x}\right| \geq\left|v_{y y}\right|$

$\eta=$ asymmetry parameter $=\frac{V_{\mathrm{xx}}-V_{\mathrm{yy}}}{\mathrm{V}_{\mathrm{zz}}}, 0 \leq \eta \leq 1$.

The energies due to the magnetic splitting are

$E_{M}=-g \mu_{n} H m_{I}$

$\mu_{\mathrm{n}}=$ nuclear magneton

$\mathrm{g}=$ gyromagnetic ratio

$\mathrm{H}=$ magnetic field magnitude.

Note that the magnetic splitting creates $2 I+1$ states while the quadrupole splitting only creates $I+1 / 2$ or $I+1$ levels. The quadrupole splitting does not lift the degeneracy of states with the same absolute value of $\mathrm{m}_{\mathrm{I}}$ : Both the excited and ground states can be split. The possible transitions are governed by the selection rule $\Delta \mathrm{m}_{\mathrm{I}}=0, \pm 1$. The magnitude of the splitting in the case of mixed magnetic and quadrupule splittlngs depends on the relative orientations of the mayntelic 
field and the electric field gradient. The splittings are illustrated in Fig. 30 for a nucleus with spin $5 / 2$ in both the excited state and in the ground state. Also shown are the seven allowed transitions for quadrupole splitting and the sixteen allowed transitions with pure magnetic splitting or mixed quadrupole and magnetic splittings. The figure shows the magnitude of the splittings to be the same in both states. This is not the general case.

Note that the splittings do not change the energy difference between the centers of gravity of the two nuclear states. This energy difference can be changed an amount $\Delta E$ if the nucleus in the excited state is different in size from the nucleus in the ground state. This shift is due to the electrostatic interaction of the nucleus with the electric field due to the electrons at the nucleus. To a first approximation, $\Delta \mathrm{E} \propto \frac{\Delta \mathrm{I}}{\mathrm{R}} \psi^{2}(0) . \quad \Delta \mathrm{E}$ is the energy shift, $\Delta \mathrm{R}$ is the change in the size of the nucleus, $\psi^{2}(0)$ is the electron chirge density at the rucleus. Ihis energy change is calied the chomleul whirl. The urifin of this terminology is clear since $\psi^{2}(0)$ is different in different chemical environments.

If there are energy shifts and/or splittings, they tend to shift the energy states of a nucleus in one environment off resonance with a gummit ray emitted by a similar nuslens in a different, environment. The ganma ray can be shifted in energy to bring it back into resonance by the simple expedient of moving the scatterer with respect to the emitter. This Doppler shifts the energy of the emitted gamma ray an amount $\Delta \mathrm{E}$. $\Delta \mathrm{E}=\mathrm{E} \mathrm{v} / \mathrm{c} . \quad \mathrm{v}$ is the Doppler velocity. The velocities normally required are quite small, a few centimers per second. The required 
Initial Quadrupole Magnetic States Splitting Splitting
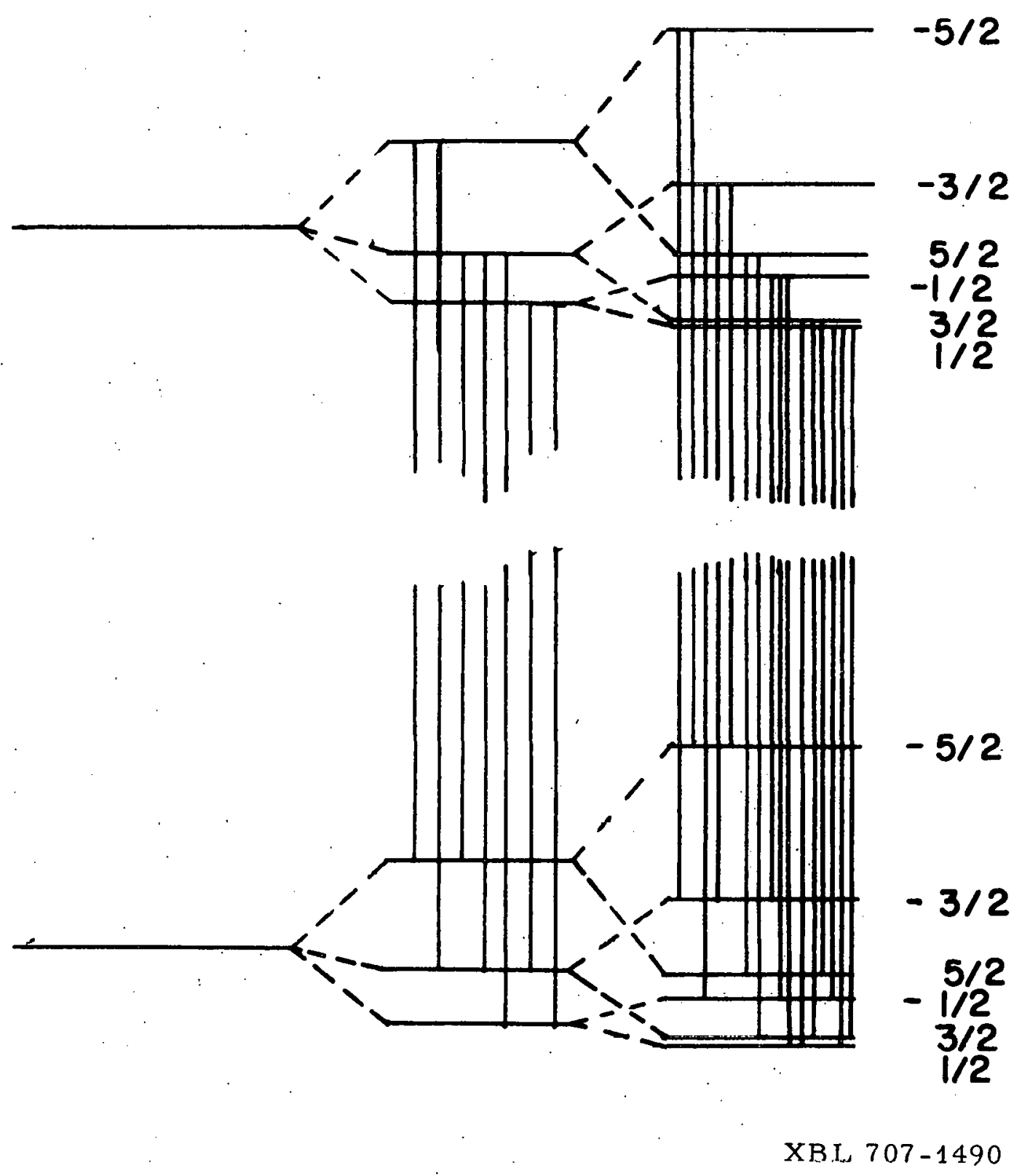

Fig. 30. Splittings of Nuclear States. 
velocities are unusually large for $D^{161}$ being as much as $\pm 25 \mathrm{~cm} / \mathrm{sec}$.

The experiment is normally performed by measuring the intensity of the radiation transmitted through the scatterer as a function of the relative velocity of the source and scatterer. A typical Mössbauer curve will show a minimum in the transmitted intensity when the resonant scattering is at a maximum. The velocity scale is linearly related to an energy scale, $\Delta \mathrm{E} . \cdot \mathrm{v}=\mathrm{c} \Delta \mathrm{E} / \mathrm{E}$.

\section{EXPERIMENTAL}

The high pressures were produced using opposed Bridgman anvils。 The flat faces of the urvils were $3 / 8 \mathrm{in}$, in diameter. The source consisted of a piece of gadolinium metal 3/16 in. in diameter and 15 mil thick which was activated by irradiating for a month at a flux of about $4.5 \times 10^{14}$ neutrons $/ \mathrm{cm}^{2} / \mathrm{sec}$ in the Arco reactor. This produced $1 \mathrm{~b}^{161}$ by the reaction $\mathrm{Gd}^{160}+\mathrm{n} \rightarrow \mathrm{Gd}^{161} \cdot \frac{4 \mathrm{~min}}{\longrightarrow} \mathrm{Tb}^{161}+\beta^{-}$. Teruiun-

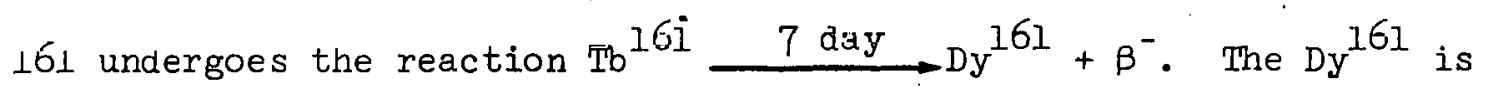
produced in an excited state. The gadolinium was purchased frum Research Chemicals Co. as $99.9 \%$ pure $20 \mathrm{mil}$ thick gadolinium foil. This was rolled to $15 \mathrm{mil}$ and the $3 / 16 \mathrm{in}$. disc punched from this. The pyrophylite ring, which surrounded the source to complete the high pressure cell, was $3 / 8$ in. o.d., $3 / 16$ in. i.d., and 20 mil thick.

The velocity spectrometer which was used was a cam-driven constant. velocity type. It is shown schematically in Fig. 31. The cam consited of two circular sections and two spiral sections with transitions regions between. The cam was so shaped that it had a constant diameter. This allowed a cam follower on each side of the cam to maintain continuous contact with the cam. The velocity is cam angle profile of 


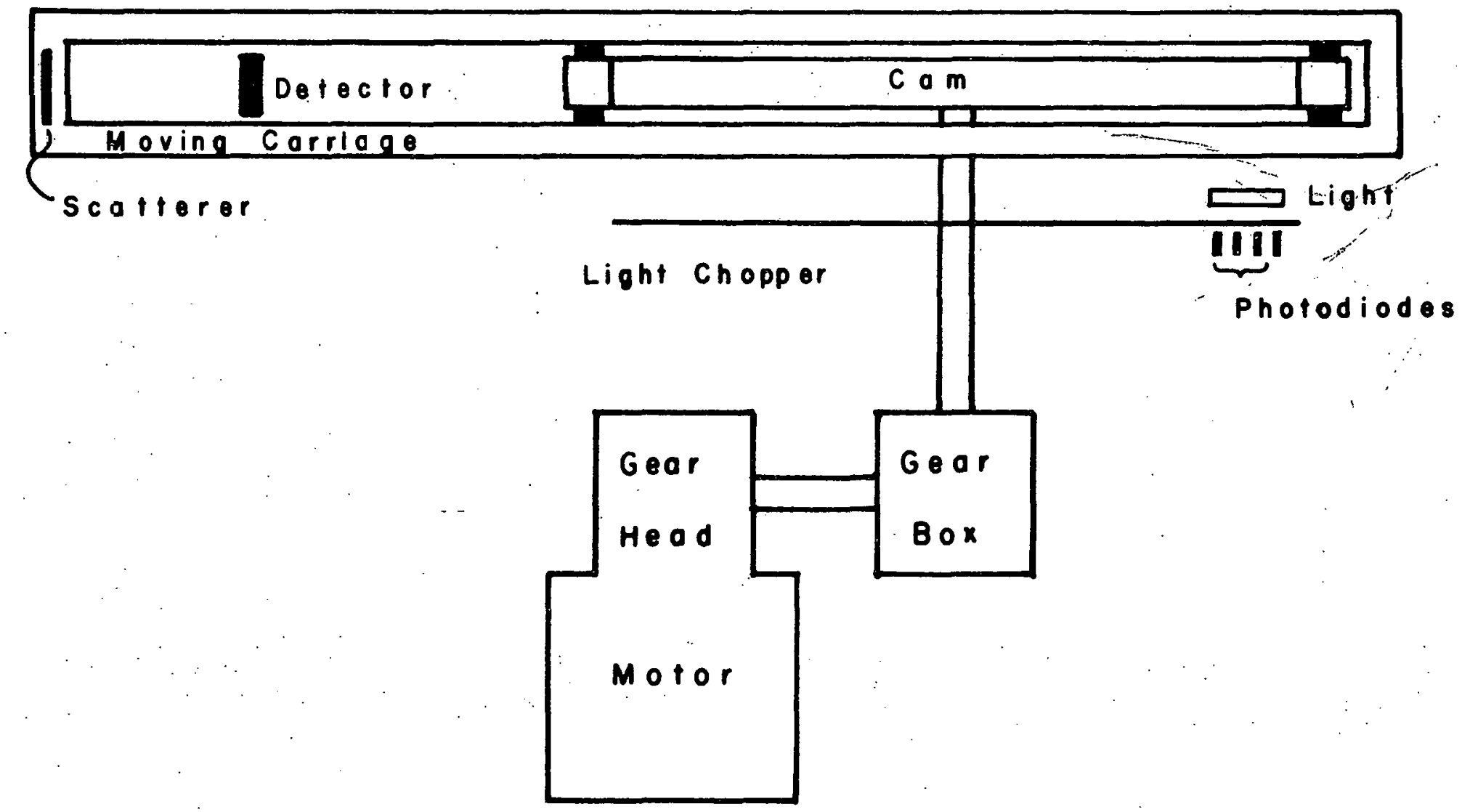

Fig. 31. Cam Driven Constant Velocity Mössbauer Spectrometer. 
the moving carriage is shown in Fig. 32. The magnitude of the constant velocity depends on the speed of rotation of the cam. In the transition regions the velocity vs angle motion of the scatterer is in the form of a section of an offset sine wave which connects smoothly with the two adjacent constant velocity sections. The total range of movement of the scatterer was 2.865 in. $(7.291 \mathrm{~cm})$.

The cam was rotated with constant velocity by a Bodine Electric Co. type NSH-55RH $1 / 4$ horsepower D.C. motor with an $18: 1$ gear head, The speed was controlled with a Minarik Electric Co. model SH-63 solid state speed control.' The speed control makes it possible to set the speed of the motor to any desired value. The control is so designed that the speed of the motor remains constant under changing torque loads. Depending on the speed range to be covsred, the output of the gear head was put through a further reduction of $50: 1$ or 10:1 or was coupled directly $(1: 1)$ to the cam. The maximum positive velocity obtainable was about $48 \mathrm{rm} / \mathrm{ser}$ with the direst coupling. It woo proportionally less with the gear reductions. The minimum velocity which was obtainable was about 0.05 of the maximum for a given gear ratio. The velocity increments between the maximum and minimum can in principle be of any size. In practice the increments cannot be set with an accuracy of better than about \pm 0.005 of the maximum. The average velocity can be measured to about $\pm 0.001 \mathrm{~cm} / \mathrm{sec}$.

The electronic set up used in the final series of experiments is shown in Fig. 33. The pulse height analyzer was used in four, onehundred channel segments in the normal pulse height analyzer mode. The photodiodes, in conjunction with the mechanjcal light chopper on 


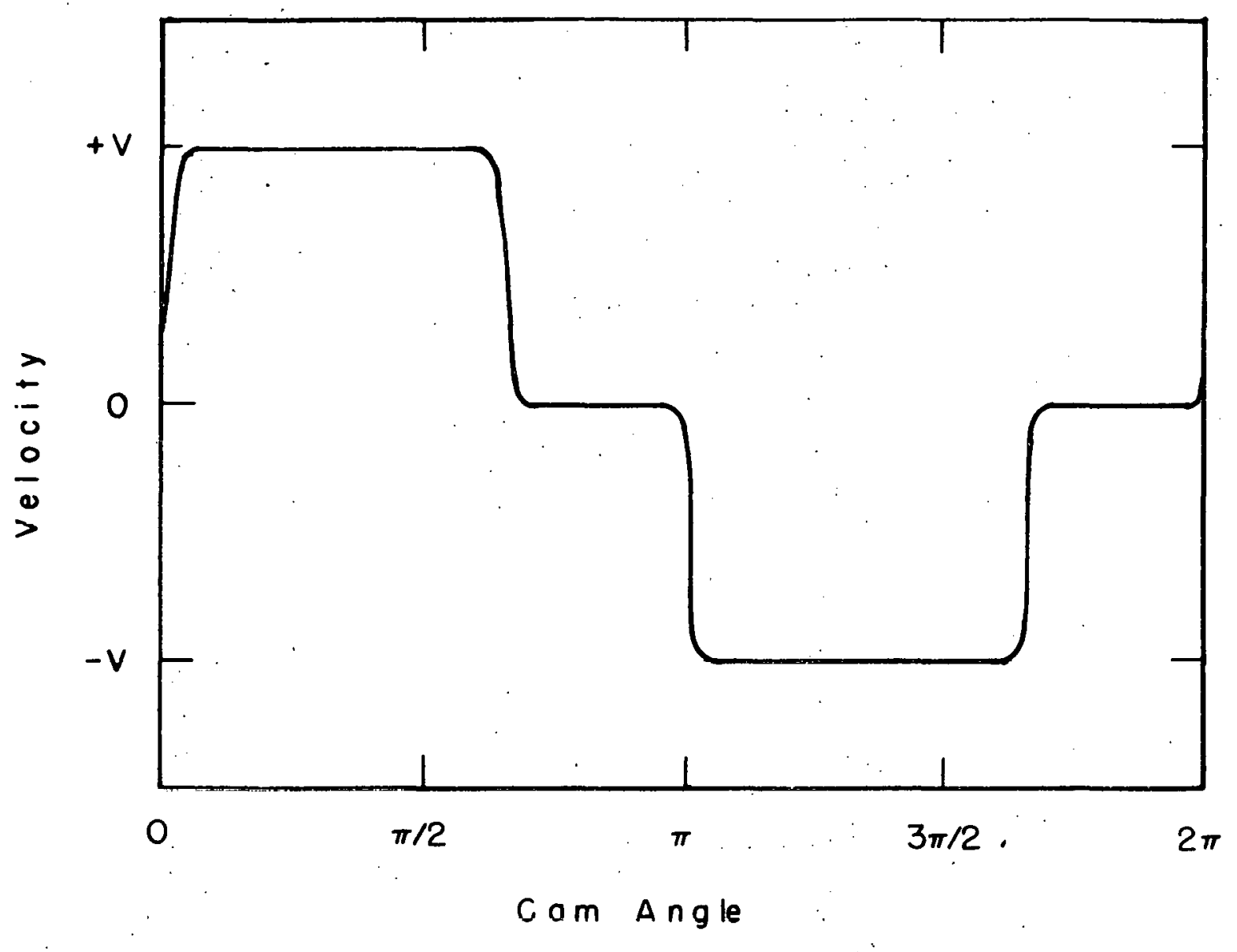

XBL $707-1488$

Fig. 32. Velocity - Cam Angle Profile of the Mössbauer Spectrometer. 


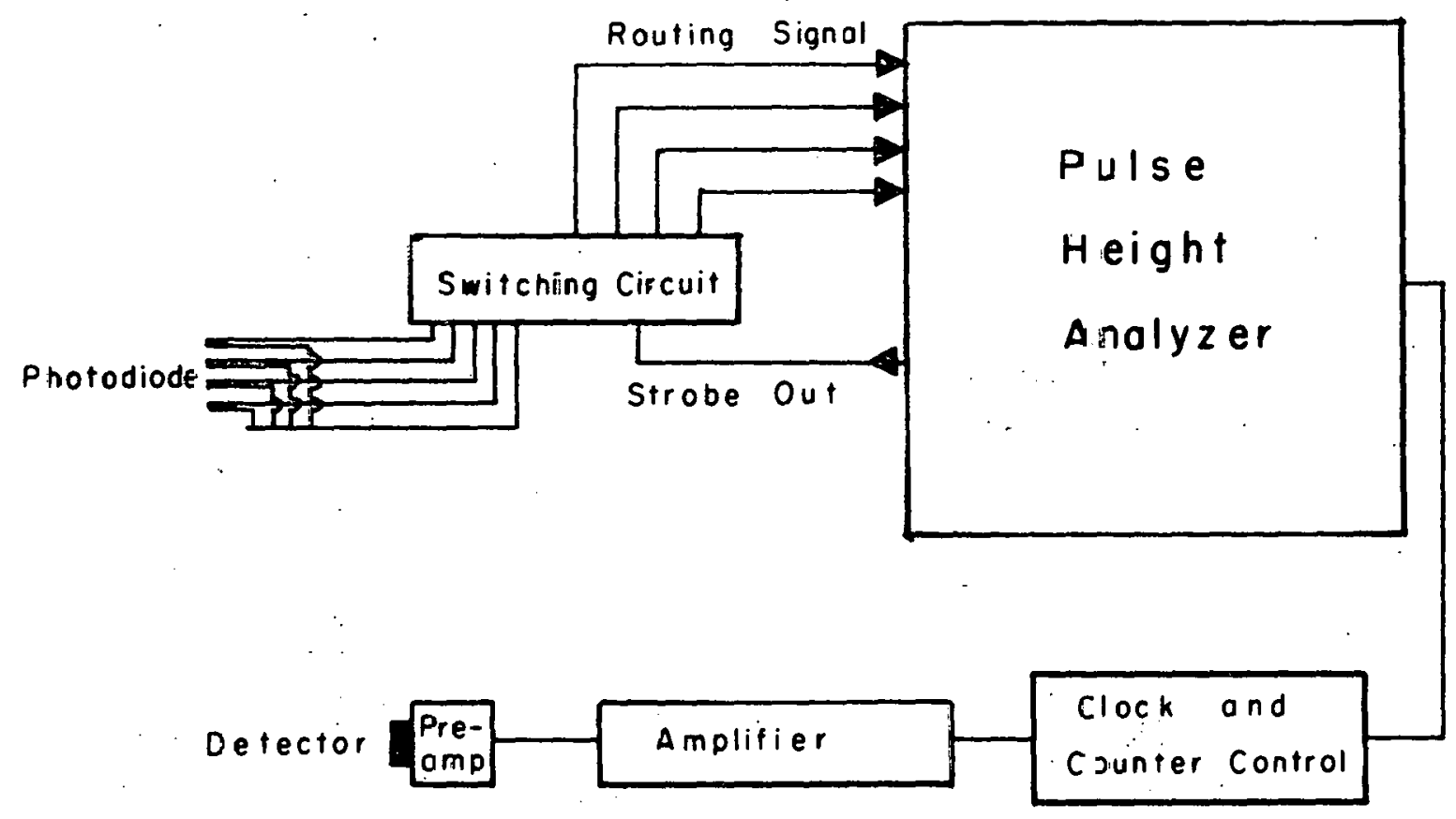

XBL $707-1487$

Fig. 33. Electronic Block Iiagram for the Mossbauer Spectrometer. 
the same shaft as the cam, were used to sense the angle of the cam. Their output was used to control in which quadrant of the pulse height analyzer the incoming pulses were counted. The four quadrants correspond to the $+V, .-V$, zero, and transition regions of the cam. The pulse height analyzer was a Victoreen model ST400UC, four-hundred channel analyzer. The switching circuit was a solid state circuit, LRL number 8S6382. The control circuit was a homemade device constructed from a number of mercury wetted reed relays which was: used, in conjunction with a microswitch activated by the cam, to start and stop the counting with the cam in the same position. It also measured the total time spent in measuring each set of velocity points and counted the number of revolutions that the cam made in that time. These data were necessary in order to calculate the average velocity of the absorber for those velocity points. The pre-amp was an IRL number 11X4730S-1D FET pre-amp with a cooled first stage. The detector was a lithium drifted silicon detector with one centimeter diameter active area made at LRL. The amplifier was a Model VI linear amplifier, LRL number $3 \times 9494$. Figure 34 shows the gamma ray spectrum obtained with this set up. This spectrum was taken through the scatterer. It is composed of the sum of the counts in the four quadrants of the PHA sumed over scveral different velocities of the scatterer. The source was at $60 \mathrm{kbars}$. The peak around channel 24 is the $26 \mathrm{keV} \mathrm{Dy}{ }^{161}$ Mössbauer gamma. The peaks in the vicinity of channels 65 to 85 are various $x$-rays and the $49 \mathrm{keV}$ gamma which is produced when $\mathrm{Dy}^{161}$ decays from the $75 \mathrm{keV}$ to the $26 \mathrm{keV}$ level. 


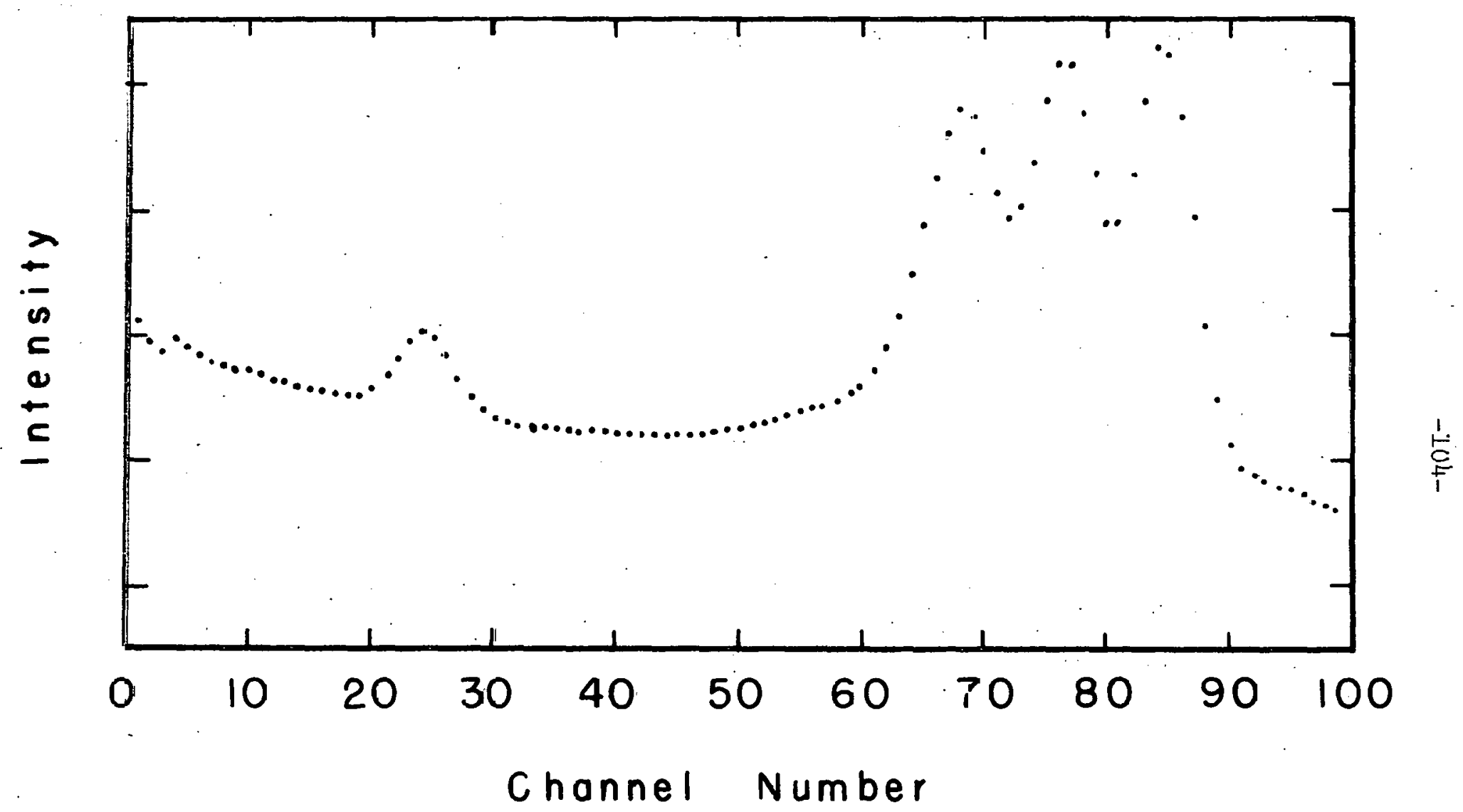

XBL 707-1486

Fig. $34 \mathrm{~Tb}^{161}$ Gamma Ray Spectrum. 


\section{RESULTS AND DISCUSSION}

Several attempts were made to duplicate Stone's experiment. ${ }^{52}$ The source was prepared in nearly the same manner as Stone's. Absorbers of the same thickness range as Stone's ( 5 to $20 \mathrm{mg} / \mathrm{cm}^{2}$ ) were prepared in an identical manner. No Mo̊ssbauer effect was observed at any presStone reported a $3 \%$ absorption at $30 \mathrm{kbar}$. In a final series of experiments a much thicker scatterer was used. It consisted of about $100 \mathrm{mg}$ of $\mathrm{Dy}_{2} \mathrm{O}_{3}$ enriched to $94 \% \mathrm{Dy}^{161}$ dispersed in about 1 gram of $\mathrm{LiF}$ and compressed into a disc one inch in diameter. The disc was annealed at about $600^{\circ} \mathrm{C}$ for about $15 \mathrm{hr} . \mathrm{Dy}_{2} \mathrm{O}_{3}$ was chosen as the material to use for the scatterer because Sklyarevsky et al. 62 had reported that this compound gave an unsplit resonance at $500^{\circ} \mathrm{C}$. Although their evidence for this unsplit resonance is questionable, it is the only report of an unsplit resonance of nearly natural linewidth with $\mathrm{Dy}^{161}$. It was hoped that if the effect a.t room temperature were great enough. it would be feasible to try to observe the effect with the scatterer at $500^{\circ} \mathrm{C}$ and thereby resolve the fine structure of the source.

The results of the measurements with the emitter at 40 and $60 \mathrm{kbar}$ are shown in Fig. 35. The point at zero velocity and relative intensity 1.000 is a fiducial point. All intensities are relative to this point. The sum of the counts in channels 22 through 27 were divided by the sum of the counts in channels 32 through 99. This ratio was calculated for the quadrants corresponding to $+V,-V$, and zero Doppler velocity. Channels 22 through 27 correspond to the $26 \mathrm{keV}$ peak. The counts in channels 32 through 99 are independent of the relative velooity of the 


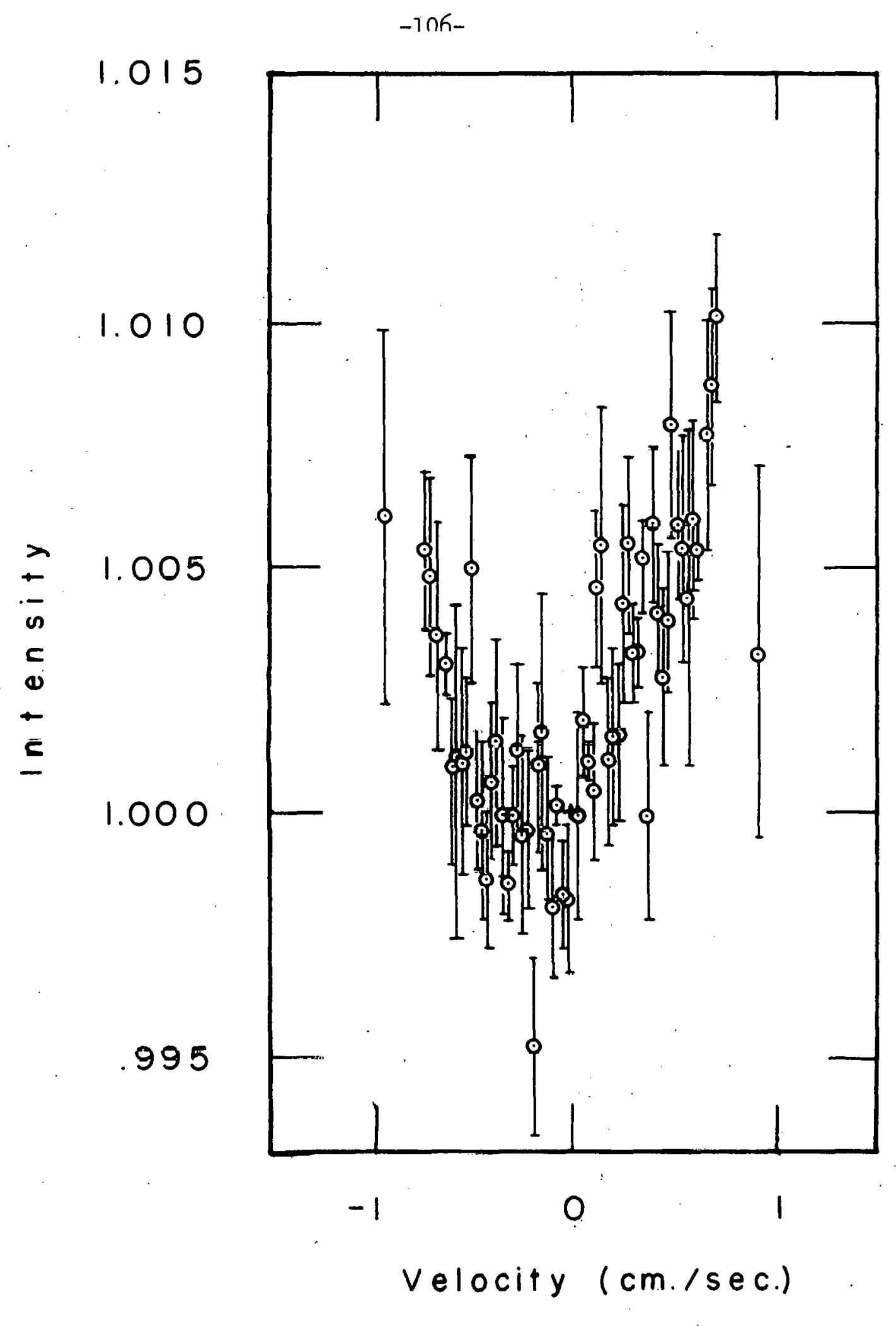

XBL $707-1485$

Fig. 35a. Dy ${ }^{161}$ Mössbauer Spectrum at 40 kilobars. 


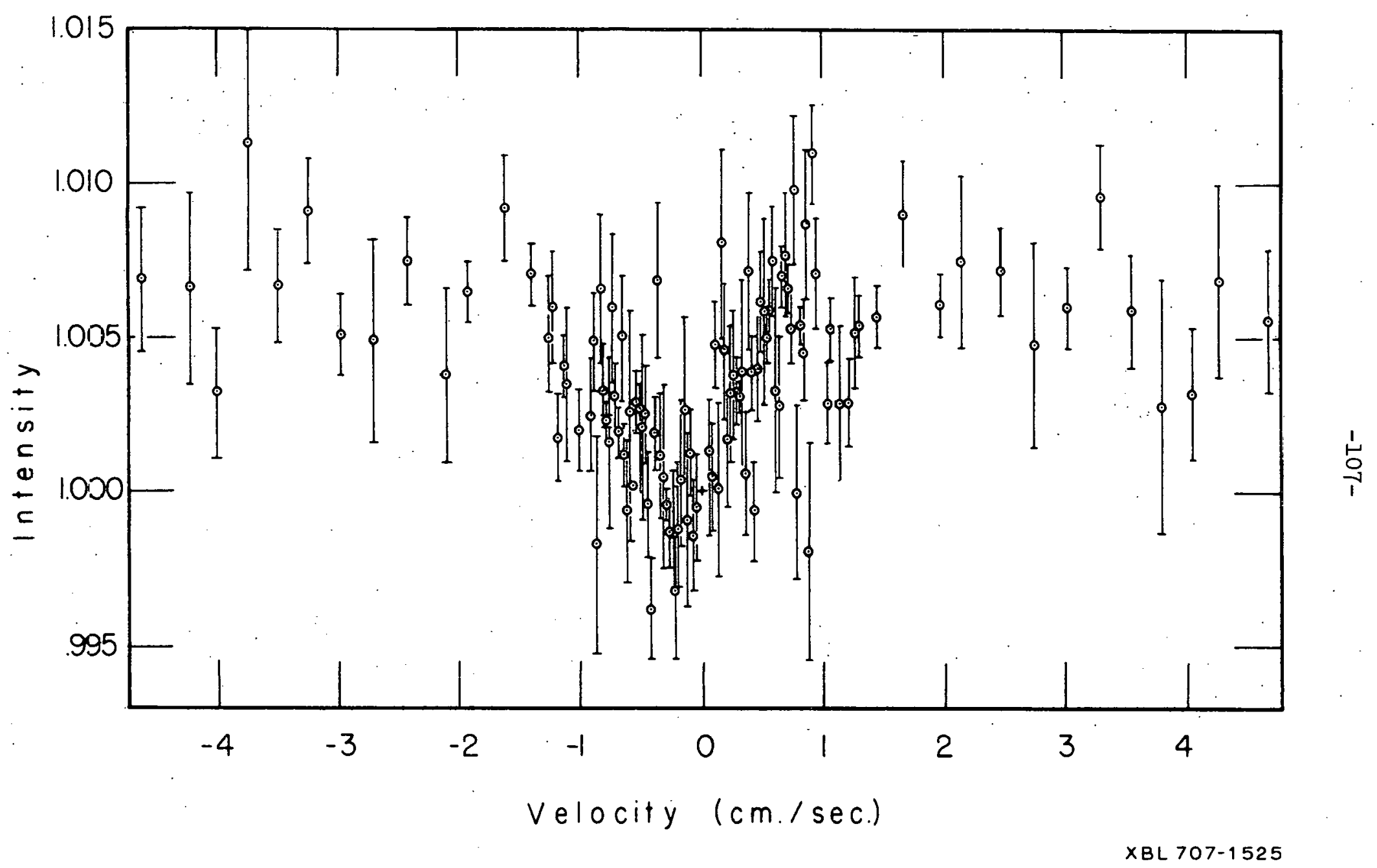

Fig. 35b. Dy ${ }^{161}$ Mössbauer Spectrum at 60 kilobars. 
scatterer and the source. The ratio, then, is a measure of the resonant scattering of the $26 \mathrm{keV}$ ganmas. The $+\mathrm{V}$ and $-\mathrm{V}$ ratios were divided by the ratio at zero velocity to obtain the points displayed in the figures. Points within a velocity range of $0.025 \mathrm{~cm} / \mathrm{sec}$ have been condensed into single points for the purposes of these figures.

There clearly is an observable Mössbauer effect with this system. The magnitude of the scattering, however, is only about $1 \%$ without background corrections. The full width at half maximum is about $0.9 \mathrm{~cm} / \mathrm{sec}$. The chemical shift is about $-0.2 \mathrm{~cm} / \mathrm{sec}$. Minus velocity indicates that the source and scatterer were moving away from each other when the scattering was a maximum. These values compare very favorably with those of ofer et al. 56 They used neutron irraduated $\mathrm{Gd}_{2}^{16 \mathrm{O}_{3}}$ at room temperature as a source and natural Dy metal $\left(300 \mathrm{mg} / \mathrm{cm}^{2}\right.$ ) at room temperature as a scatterer. The environment of Dy in $\mathrm{Gd}_{2} \mathrm{O}_{3}$ should be similar to Dy in $\mathrm{Dy}_{2} \mathrm{O}_{3}$, and Dy in Dy metal should be similar to Dy in Gd metal above the Curie temperature. The crystal structures are identical and Dy is nearly the same size as Gd.

The effect appeared to be too small to make it profitable to perform more detailed investigations with the same electronics and scatterer. The quadrupole splittings might be observed if a good single line scatterer could be found. There should be no more than seven lines wi.th nnt.y quadrupole splitting in the source since the $\mathrm{Dy}^{16 \mathrm{I}}$ nucleus is known to have spin 5/2 in both the excited and ground states. Since the quadrupole moment is known to be nearly the same in both the excited and ground state, ${ }^{53-8}$ the three $\Delta m_{I}=0$ transitions will be nearly the same energy. This meane that only five lines will be ouserved. 


$$
-109-
$$

Since the natural linewidth of $\mathrm{Dy}^{161}$ is only about $0.07 \mathrm{~cm} / \mathrm{sec}$, five separate lines could probably be resolved with a single line scatterer of natural linewidth if the outermost lines are split from each other by as much as $0.5 \mathrm{~cm} / \mathrm{sec}$. Since $\mathrm{Dy}_{3} \mathrm{O}_{3}$ at $500^{\circ} \mathrm{C}$. would give too small of an effect and no other possibilities were known, the experiment was terminated. 


\section{CRYSTALILINE PHOSPHOROUS}

A. INTRODUCTION

A considerable amount of work has been done with crystalline or black phosphorous since Bridgman's discovery of it in 1914.63 This has resulted in an extensive body of information about it. Most of this information is at lower pressures than are available today. It was quick $\perp$ discovered to have fair electrical conductivity and a nega-

tive temperature coefficient of resistivity at atmospheric pressure. 63,64 This is characteristic of a semiconductor. Later work revealed that the crystalline phase is thermodynamically stable with respect to the other known forms of elemental phosphorous at room temperature. 65 The other known forms are all amorphous. Its rather complicated atmospheric pressure crystal structure was elucidated by Hultgren et al. ${ }^{6}$ His calculated density of $2.69 \mathrm{gm} / \mathrm{cm}^{3}$ compared very f'avorably with measured values. $63,67-9$

Other high pressure phases of phosphorous were reported from time to time. ${ }^{70-2}$ While investigating the crystal structure of the high pressure phases of phosphorous, Jamieson found a previously unknown phase change of phosphorous from the A7 (arsenic) type of structure to Eimple cublc at $111 \pm 9 \mathrm{kbar} .73$ jince pliospluruus in a simple cubic structure must be metallic in nature, it appeared as though the phase transition might be accompanied by a large change in the electrical resistivity. It was, therefore, decided to measure the resistance of a phosphorous sample up to $130 \mathrm{kbar}$. This is somewhat hig her than previous measurements had gone. 


\section{B. THEORY}

It is very difficu:. to calculate the electrical conductivity of a solid from a-priori assumptions. Normally certain empirical parameters are used in the description of the phenomenon. In the Free Electron Approximation, the electrical conductivity, $\sigma$, is $n e^{2} \tau / m$. $n$ is the number of free electrons per unit cell, e is the charge of an electron, $m$ is its mass, and $\tau$ is the relaxation time, which is assumed to be the same for all conduction electrons. $\tau$ is not a quantity which is easily calculated. Depending on the size, purity, and temperature of the solid, the effect on $\tau$ of one or more of the following phenomena may have to be considered: impurity scattering, boundary scattering, phononelectron scattering, electron-electron scattering, and perhaps others. If more than one conduction band must be considered, the expression for the conductivity becomes; $\sigma=n_{1} e^{2} \tau_{1} / m_{1}+n_{2} e^{2} \tau_{2} / m_{2}+\cdots$ The numbers refer to different bands. $\eta_{1}$ is the effective number of conduction electrons in the first band, and $m_{1}$ is the effective mass. Only those electrons which lie on a section of the free Fermi surface and those electrons beneath them are effective in the electrical conductivity. Those electrons on the Fermi surface at a zone boundary cannot be excited to other states by an electric field of ordinary magnitude, except in certain semiconductors (Zener breakdown). The quantities $\sigma$, neff

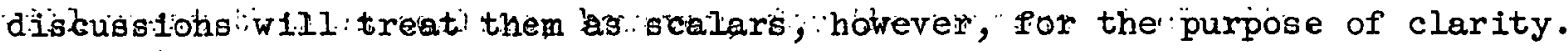

For simple metäls at ordinary temperatures, $n_{\in f f}$ and $m_{\text {eff }}$ are relatively independent of pressure. Therefore; $\partial \sigma / \partial \mu$ depends primarily 
on $\partial_{\tau} / \partial_{P} . \quad \tau$ is dominated by the electron-phonon scattering at ordinary temperatures. Since the Debye temperature increases with pressure, the number of phonons decreases, and the electron-phonon scattering decreases with pressure. This leads to an increasing $\tau$ and, therefore, an increasing $\sigma$. The conductivity of a simple metal, therefore, should show a slight increase with increasing pressure at constant temperature.

The pressure dependence of the conductivity of a semiconductor usually depends primarily on $\partial_{n_{1}} \partial p$, and for $\partial_{n_{2}} \partial \partial p$. At least two bands must be considered for semiconductors. These derivatives depend on the pressure derivative of the band gap. No simple rules exist for predicting how the band gap of a semiconductor will change with pressure. The conductivity of a semiconductor may either increase or decrease with pressure. 
C. RESULTS

The high pressures for this experiment were produced using Bridgman anvils. For resistance measurements the substance of interest occupies only a small percentage of the available volume. The balance of the volume is filled by silver chloride which provides an electrically insulating, quasi-hydrostatic pressure transmitting medium.

In all of the phosphorous resistance measurements, the black phosphorous was produced in sito from red phosphorous. * The transition takes place at about 90 kbars in the system which was used. This agrees with the transition pressure which was observed by Bridgman ${ }^{72}$ in the course of compressibility measurements on red phosphorous. With a great deal of shear force the transition may occur below $50 \mathrm{kbar}$ at room. temperature. ${ }^{74}$ In practace the application of an electric field of $135 \mathrm{~V}$ from three $45 \mathrm{~V}$ "B" batteries would cause the transition to occur as low as 50 to $60 \mathrm{kbar}$ in the system which was used.

An initial series of relatively crude experiments was performed. These experiments failed to reveal a pressure discontinuity as great as $1 \%$, but they did suggest a possible change in the slope of the resistancepressure curve at $110 \pm 10 \mathrm{kbar}$. A more careful series of experiments was, therefore, performed. All of the new samples included an internal thermocouple and an internal pressure standard. The pressure standard consisted of a length of blsmuth wire. Bismuth has three well known phase transitions under pressure which are easily. seen with resistance measurements. The Bi I-II, II-III, and III-V transitions are assumed

\footnotetext{
B \& A Amorphous Phosphorous, Red Powder, Code 2062.
} 
to occur at $25.4,27.0$ and $88 \mathrm{kbar}$ respectively. ${ }^{18-20}$ All resistances were measured using the four-lead technique, which eliminates the effect of contact resistance. The high pressure cell was surrounded by a bath of isopropyl alcohol for this series of experiments. During the constant temperature measurements the bath was thermostated at about $28^{\circ} \mathrm{C}$ and held constant to better than $0.1^{\circ} \mathrm{C}$. Later temperature coefficient measurements showed that a change in temperature of $0.7^{\circ} \mathrm{C}$ woml result in a resistance change of the phosphorous of about $0.01 \%$. For measurements of resistance as a function of temperature the bath was cooled with dry ice or heated with a resistance heater.

A number of different samples were built which differed in the details of construction, but which were basically the same in concept. One of these is shown in exploded form in Fig. 36. The phosphorous sample was formed by carefully filling a depression in the silver chloride disc with red phosphorous. The depressinn was made by preesing a hoop of $5 \mathrm{mil}$ wire about $3 \mathrm{mil}$ into the silver chloride, and then removing the wire. All lead ont wires were 5 mil platinum wires, cxcept. for the thermocouple wires which were 36 gauge chromel A and alumel wire.

Although a number of samples were built, only two yielded extensive results. There were experimental difficulties with the others. The partial results from the other did agree with the more complete results from the two good samples.

Resistance measurements on the first good sample:were precise to about $0.1 \%$. 'The measuring current was $100.0 \mathrm{~mA}$. These measurements revealed that the resistance of the sample did not come to a constant 


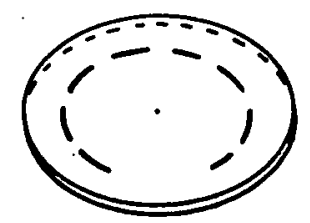

5 mil $\mathrm{AgCl}$ with Phosphorous

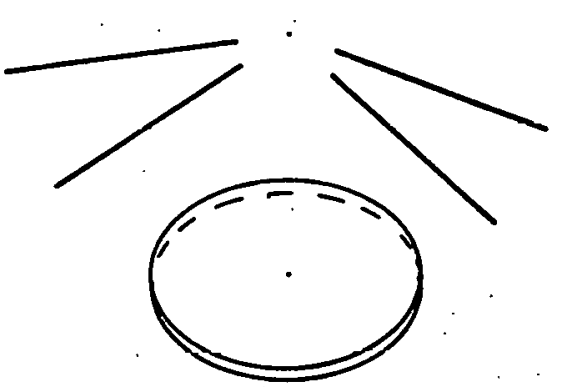

5 mil Pf leads

$2 \mathrm{I} / 2 \mathrm{mil} \mathrm{AgCl}$

Chromel A-Alumel Thermocouple

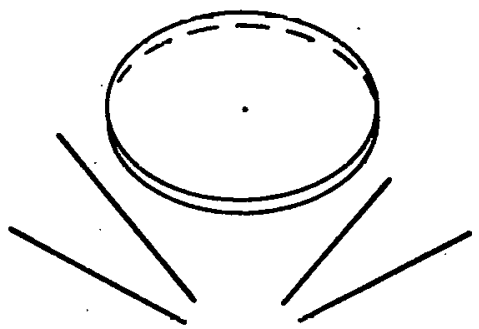

$21 / 2 \mathrm{mil} \mathrm{AgCl}$

5 mil Pt leads

13 mil Bismuth wire hoop

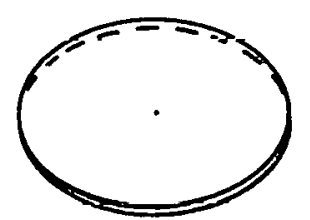

$5 \mathrm{mil} \mathrm{AgCl}$

Fig. 36. Exploded View of a High Pressure Sample Used for the Fhosphorous Resistance Measurement. 
value even several hours after a pressure change. After increasing the pressure the resistance would decrease with time, and after decreasing the pressure the resistance would increase with time. The sample was held at 122 kbar until the resistance became constant (about 1 day). Then the resistance was measured as a function of temperature. These data are shown in Fig. 37. A thermocouple reading of $0.6 \mathrm{mV}$ corresponds to about $28^{\circ} \mathrm{C}$. The reference junction was at the ice point. There is a oonsiderable amnunt nf temperature hysteresis apparent in the data. Thịs is probably due to a temperature gradient between the sumple and the thermocouple. The cooling and heating cycles were both done quite quickly: It is surprising that the gradient would be so large, as the sample and thermocouple were not more than $1 / 4 \mathrm{in.}$ apart. Only the sign of the temperature coefficient, however, is of interest, and this cun ve detemined unambiguous $\perp y$ in splte of the lemperature hystcrlusu. Th resistance was measured with the current flowing first in one direction and then the other. The average of the two measurements is shown in the figure. This procedure eliminates the effects of thermal EMF's.

Careful resistance-pressure measurements were made with the second good sample. The resistance could be measured with the apparatus which was used with this second sample to a precision of $0.01 \%$. The uncertainty introduced by the time dependent drift of the rcsistance is greater than this. The measuring current that was used was about $0.4 \mathrm{~mA}$. The experiment was performed in the following manner. After a pressure change, the pressure was held constant until the resistance of the phosphorous was constant to within $0.03 \%$ over a period of several hours. l'hen another pressure change was made. It took about six weeks to collect the data shown in Fig. 38 using this method. 


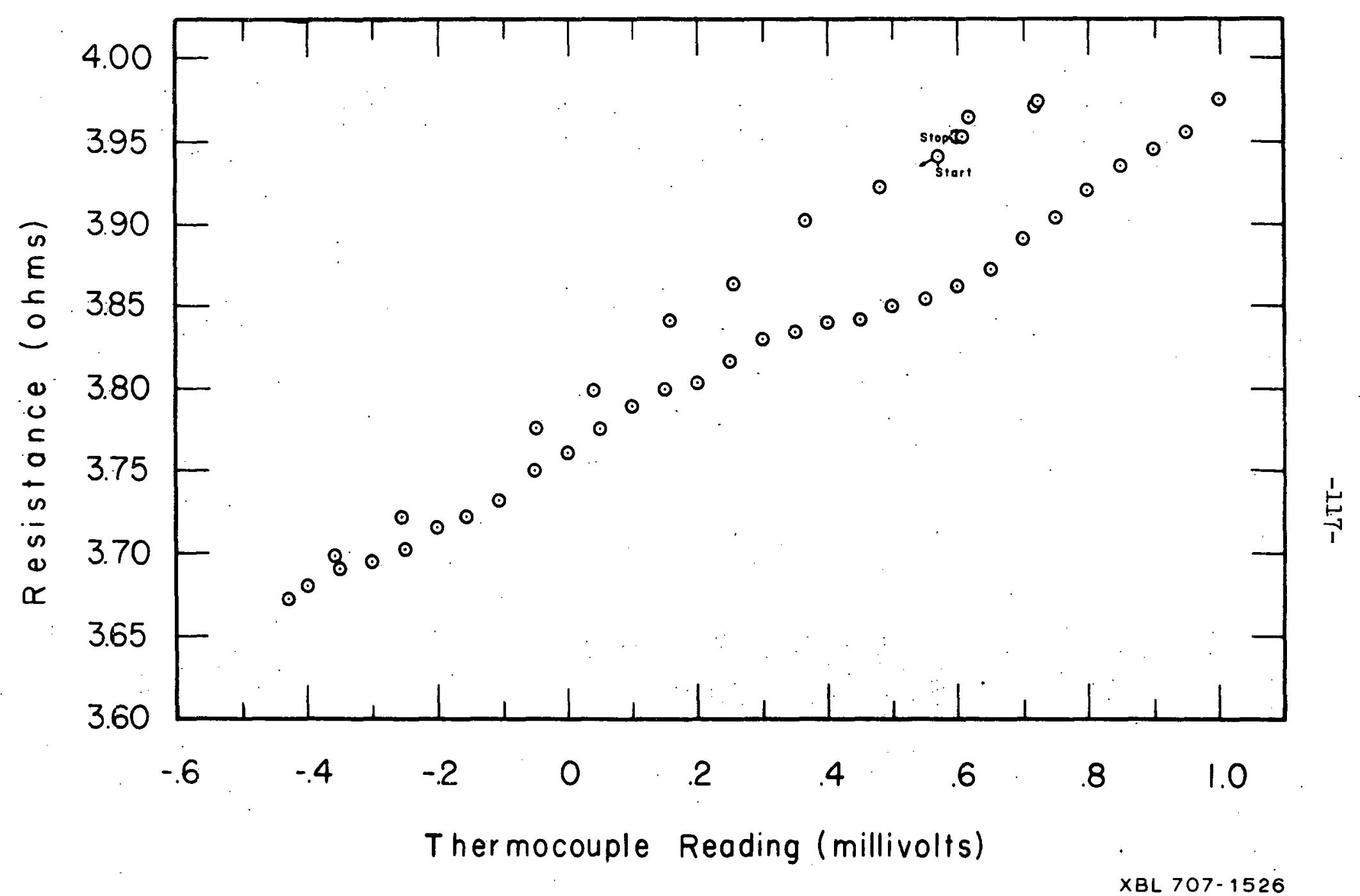

Fig. 37. Resistance of Crystalline Phosphorous as a Function of Temperature at 122 kilobars. 


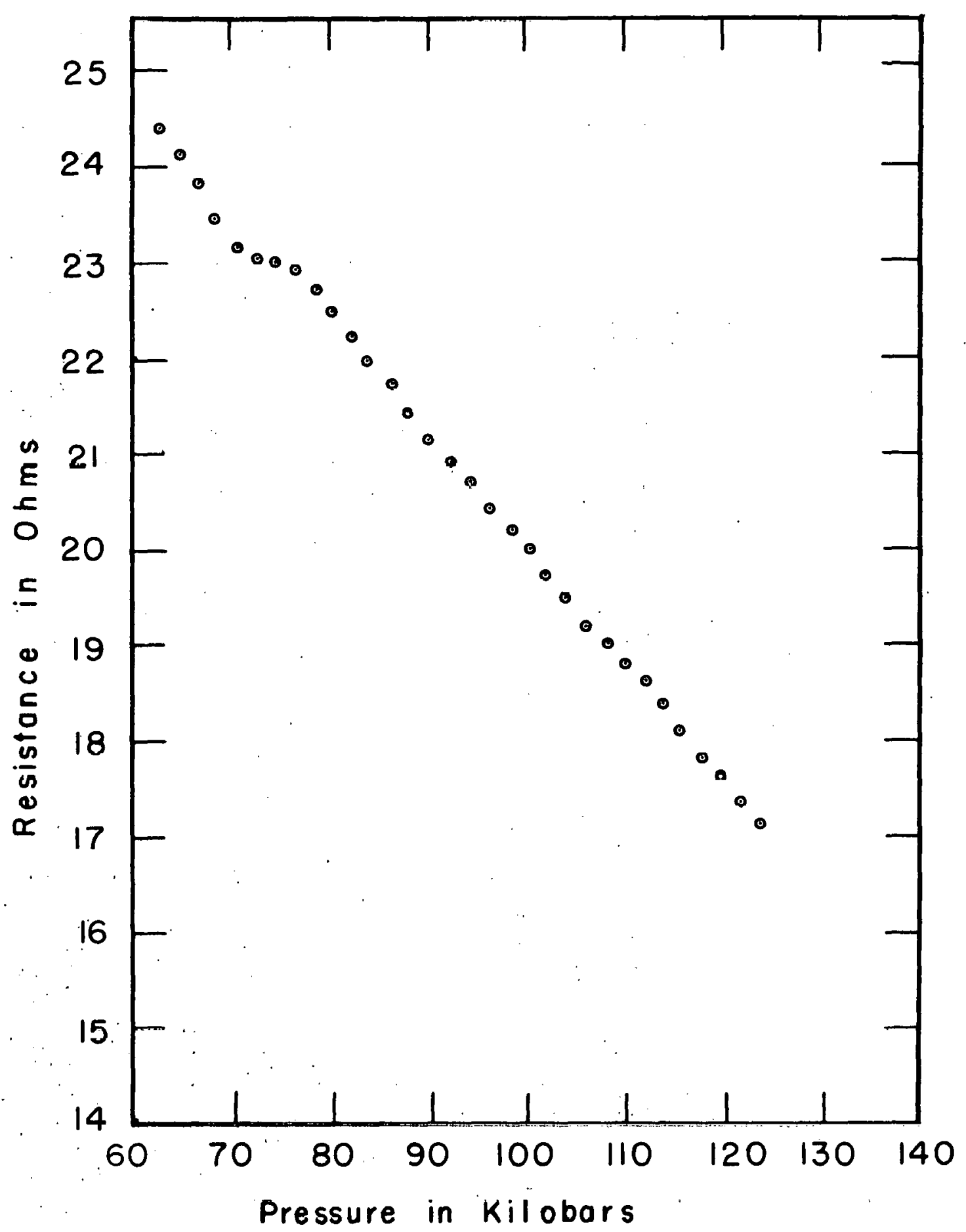

$X B], 707-1483$

Fig. 38. Resistance of Crystalline Phosphorous as a Function of Pressure at $28^{\circ} \mathrm{C}$. 


\section{DISCUSSION}

It is known that crystalline phosphorous above $80 \mathrm{kbar}$ has the A7 (arsenic) structure. 73 This is a rhombohedral structure with a basis. The rhombohedral angle, $\alpha$, at $83 \mathrm{kbar}$ is $57.25^{\circ}$, and $a_{r}=3.524 \AA .73$ The basis vectors are $(0,0,0),(2 u, 2 u, 2 u)$ in terms of the lattice vectors. $u$ is between 0.21 and 0.22 at $83 \mathrm{kbar}$. According to Jamieson this becomes simple cubic with a $=2.37 \AA$ at about 111 kbar. ${ }^{73}$ The simple cubic structure is just a special case of rhombohedral with $\alpha=60^{\circ}, u=1 / 4$, and as such represents only a slight distortion of the lower pressure rhombohedral structure. This distortion, however, may have profound effects on the electrical properties.

Phosphorous has five conduction electrons per atom. The rhombohedral structure has two atoms per unit cell. Each band, or zone, is capable of holding two electrons per primitive unit cell. If the band gaps in rhombohedral phosphorous were very large, the ten electrons per unit cell would exactly fill the first five bands and rhombohedral phosphorous would be an insulator. With smaller band gaps it would be a semiconductor or metal. Simple cubic phosphorous, on the other hand, has only one phosphorous atom per primitive unit cell, and therefore need only accommodate five electrons per unit cell in the band structure. Since, regardless of the energy gaps, one band must be at least half empty, simple cubic phosphorous must be a metal. A room temperature resistivity on the order of $10: \mu \Omega-\mathrm{cm}$ for $\mathrm{sc}$ phosphorous is not out of line with this reasoning. 
The resistance curve shown in Fig. 38 reveals no real evidence of a phase transition at or about $111 \mathrm{kbar}$. There does appear to be a small discontinuity in the resistance curve between the points at $113.8 \mathrm{kbar}$ and $115 . \mathrm{l} \mathrm{kbar}$, but it is not clear that this is outside of the experimental error due to the time dependent drift of resistance. This experimental set up is very poor for determining resistivity because the cross sectional area of the specimen is very uncertain. However, an order of magnitude estimate can be made, and this gives a figure of about 100 moremis. This is an order of magnitude greater than what is expected for simple cubic phosertorous. It can be said, then, that the resistivity measurements give no support for the existence of simple cubic phosphorous in the pressure interval which was covered in this experiment. A later cumpression to $130 \mathrm{kbar}$ also failed to show any evidence of a phase transition.

The pressure dependence of the resistance also appears to be greater than what would be expected for a good metal. As discussed earlier, the pressure dependence of resistivity of a good metal at ordinary temperatures depends mainly on the pressure dependence of the Debye temperature. This in turn depends mainly on the compressibility of the metal. Compressibility measurements by Bridgman ${ }^{72}$ reveal that the compressibility alone is not great enough to account for the ohserver pressure dependence of resistivity. 'This implies that the eflective number of electrons is increasing. This is the kind of behavior that is expected for a poor metal in which the band overlap is increasing with pressure. This is consistent with the rhombohedral form of phosphorous, but not with the simple cublc form. 
In summary, if simple cubic phosphorous does exist it must be at a pressure higher than 130 kbars on the scale used here. This pressure scale is based on the bismuth III-V transition occurring at 88 kbar on compression. Jamieson's pressure scale is based on the compressibility of $\mathrm{NaCl}$ as measured by Bridgman. Therein may lie the discrepancy between Jamieson's observation of simple cubic phosphorous at $111 \pm 9$ kbar and the failure of the present work to observe it at pressures as high as $130 \mathrm{kbar}$. 


\section{ACKNOWLEDGMENTS}

The author would like to thank Professor George Jura whose guidance throughout my stay in Berkeley was invaluable.

He would also like to thank Mr. Duane Newhart whose aid in constructing most of the physical apparatus used in these experiments was deeply appreciated.

Special mention should be made of the many interesting and useful diacuodiono with hio oolleagues, Dr. Walter Stark, Ir., Mr. Mnnal. Raimondi, and Dr. James Burton. Also discussions with Mr. Albert Yee, Mr. Tang-Hua Chen, and Mr. Che-Kuang Wu were helpful.

This work was performed under the auspices of the U.S. Atomic Energy Commission. 
REFERENCES

1. J. D. Barnett, C. D. Bosco, Rev. Sci. Inst. 38, 957 (1967).

2. C. A. Swenson, in Solid State Physics, Vol. II (Academic Press, New York, . 1960) p. 50.

3. J. J. Burton, I. Relaxations in a Moleoular Crystal, II. The Fermi Momentum of Aluminum form 0 to 100 Kilobars, (Ph. D. thesis), UCRL-17479, April 1967.

4. H. T. Hall, L. Merrill, Inorg. Chem. 2, 618 (1963.).

5. H. T. Hall, J. D. Barnett, L. Merrill, Science 139, 111 (1963).

6. P. C. Souers, G. Jura, Science 140, 481 (1963); P. C. Souers, Effect of High Pressure on the Electrical Resistance of $B i, Y b$, and Dy, (Ph. D. thesis), UCRL-11220, Jan. 1964.

7. I. Katz, A. S. Penfold, Rev. Mod. Phys. 24, 28 (1952).

8. R. E. Bell, R. I. Graham, Phys. Rev. 90, 644 (1953).

9. S. DeBenedetti, C. E. Cowan, W. R. Konneker, H. Primakoff, Phys. Rev. 77, 205 (1950).

10. R. L. Garwin, Phys. Rev. 91, 1571 (1953).

11. G. E. Lee-Whiting, Phys. Rev. 97, 1557 (1955).

12. D. Stroud, H. Ehrenreich, Phys. Rev. 171, 399 (1968).

13. J. P. Corbotte, S. Kahana, Phys. Rev. 139, A213 (1965).

14. B. Stephan, J. S. Plaskett, Phys. Rev. 112, 1877 (1958).

15. A. Hätamo, H. Kanazawa, Y. Migura, Prog. Theoret. Phys. (Kyoto) 34, $875(1.965)$.

16. S. Kahana, Phys. Rev. 117, 123 (1960).

17. E. Danie1, S. H. Vasko, Phys. Rev, 120, 2041 (1960). 
18. P. W. Bridgman, Phys. Rev. 57, 235 (1940).

19. G. C. Kennedy, P. N. LaMöri, in Progress in Very High Pressure Research (John Wiley and Sons, Inc., New York, 1961), p. 304.

20. I. F. Vereschchagin, E. V. Zukova, I. P. Buinova, Sov. Phys. DokI. 11, 585 (1967).

21. C. H. Chang, C. S. Cook, H. Primakoff, Phys. Rev. 90, 544 (1953).

22. P. S. Takhar, Phys. Rev. 157, 257 (1967),

23. F. Rohrlich, B. C. Carlson, Phys. Rev. 93, 38 (1954).

24. H. H. Seliger, Phys. Rev. 100, 1029 (1955).

25. J. S. Marshall, A. G. Ward, Can. J. Res. Al5, 39. (1937).

26. R. W. Varder, Phil. Mag. 29, 725 (1915).

27. P. A. Macklin, L. J. Lidofsky, C. S. Wu, Phys. Rev. 78, 318A (1950).

28. B. T. Wright, Phys. Rev. 90, 159 (1953).

29. C. Wong, Phys. Rev. 95, 761 (1954).

30. A. T. Stewart, J. B. Shand, Bull. Am. Phys. Soc., Sec. II, 10, $21(1965)$.

31. P. W. Bridgman, Proc. Am. Acad. Sci. 76, 55 (1948).

32. J. C. Jamieson, in High Pressure Measurement (Butterworth Inc., Washington, 1963), p. 389 .

33. I. Ya. Dekhtyar, V. S. Mikhalenkov, S. G. Sakharava, Dokl. Akad. Nauk. SSSR 168, 785 (1966), translated in Sov. Phys. Dokl. 11, $537(1.966)$.

34. J. H. Kusmiss, A. T. Stewart, Adv. Phys. 16, 471 (1967).

35. J. K. Mackenzie, G. F. O. Langstroth, B. T. A. McKee, C. G. White, Can. J. Phys. 42, 1837 (1964).

36. Tuan-Wu Chen, Ing-Jiunn Ma, Ji-Peng Chien, Chirl. J. Phys. 2, 31 (1967). 
37. N. W. Ashcroft, Phil. Mag., Ser. 8, 8, 2055 (1963).

38. P. J. Melz, Phys. Rev. 152, 540 (1966).

39. G. Lang, S. DeBenedetti, Phys. Rev. 108, 914 (1957).

40. P. W. Bridgman, Proc. Am. Acad. Arts Sci. 74, 425 (1942);

Phys. Rev. 60, 351 (1941).

41. D. R. Stevens, J. Phys. Chem. Solids 25, 423 (1964).

42. G. S. Anderson, S. Legvold, F. H. Spedding, Phys. Rev. 111, 1257 (1958).

43. J. M. Lock, Proc. Phys. Soc.70B, 476 (1957).

44. J. Crowell, V. E. Anderson, R. H. Richie, Phys. Rev. 150, 243 (1966).

45. See any standard text on the subject, or A. J. Freeman, in

Quantum Theory of Atoms, Molecules, and the Solid state,

(Academic Priess, New York, 1966), p. 369.

46. L. Koester, H. Mair-Leibnitz, K. Schmeiser, Z. Angew. Phys. 5, $9(1953)$.

47. Handbook of Chemistry and Physics, 43rd Edition (The Chemical

Rubber Publishing Co., Cleveland, 1961) p. 1522.

48. Ibid, p. $2722-3$.

49. J. L. Rodda, M. G. Stewart, Phys. Rev. 131, 255 (1963).

50. D. R. Stevens, J. Phys. Chem. Solids 26, 943 (1965).

51. A. Jayarama, Phys. Rev. 135, Al056 (1964).

52. J. A. Stone, The Influence of High Pressures on the Mössbauer Effect in Dysprosium-161, (Ph. D. thesis), UCRL-10630, April 1963.

53. R. Bauminger, S. G. Cohen, A. Marinov, S. Ofer, Phys. Rev. Letters 6, $467(1961)$.

54. A. J. F. Boyle, H. E. Hall, Rept. Prog. Phys. 25. 441 (1962). 
55. E. Bauminger, L. Grodzins, A. J. Feeman; Rev. Mod. Phys. 36, $392(1964)$.

56. S. Ofer, M. Rakavy, E. Segal, B. Khurgin, Phys. Rev. 138, A241 $(1965)$.

57. I. Nowik, H. H. Wickman, Phys, Rev. 140, A869 (1965).

58. I. Nowik, J. H. Wernick, Phys. Rev. 140, Al31 (1965).

59. H. Frauenfelder, The Mössbauer Effect, (W. A. Benjamin Inc., New York, 196亏).

60. G. K. Wertheim, Mössbauer Effect: Principles and Applications, (Academic Press, New York, 1964).

61. H. Lustig, Am. J. Phys. 29, 1 (1961).

62. V. V. Sklyarevsky, K. P. Aleshin, V. D. Gorobchenco, J. J. Lukashevich, B. N. Samoihov, E. P. Stepanov, Phys. Letters 6 , 157 (1963).

63. P. W. Bridgman, J. Am. Chem. Soc. 36, 1344 (1914).

64. P. W. Bridgman, Proc. Am. Acad. Sci. 56, 61 (1921).

65. L. Pauling, M. Simonetta, J. Chem. Phys. 20, 29 (1952).

66. R. Hultgren, N. S. Gingrich, B. E. Warren, J. Chem. Phys. 3, $3517.1 .935)$.

67. M. Marckwald, K. Helmholz, Z. Anorg. Allgem. Chem. 124, 81 (1922).

68. P. L. Gunther, P. Geselle, W. Rebentisch, Z. Anorg. Allgem. Chem. $250,373(1943)$

69. R. W. Keyes, Hhỹs. Kev. 92,500 (1953).

70. P. W. Rridgman, Phys. Rev. 45, 844 (1934).

71. R. B. Jacobs, J. Chem. Phys. 5, 945 (193.7).

72. P. W. Bridgman, Proc. Am. Acad. Sci. 76, bs (1448). 


\section{$-127-$}

73. J. C. Jamieson, Science 139, 1291 (1963).

74. P. W. Bridgman, Phys. Rev. 48, 825 (1935).

75. D. B. McWhan, T. M. Rice, P. H. Schmidt, Phys。 Rev. 177, 1063 (1969). 
This report was prepared as an account of Government sponsored work. Neither the United States, nor the Commission, nor any person acting on behalf of the Commission:

A. Makes any warranty or representation, expressed or implied, with respect to the accuracy, completeness, or usefulness of the information contained in this report, or that the use of any information, apparatus, method, or process disclosed in this report may not infringe privately owned rights; or

B. Assumes any liabilities with respect to the use of, or for damages resulting from the use of any information, apparatus, method, or process disclosed in this report.

As used in the above, "person acting on behalf of the Commission" includes any employee or contractor of the Commission, or employee of such contractor, to the extent that such employee or contractor of the Commission, or employee of such contractor prepares, disseminates, or provides access to, any information pursuant to his employment or contract with the Commission, or his employment with such contractor. 
